NASA Technical Memorandum 102334

ICOMP-89-21

\title{
Hopf Bifurcation in the Driven Cavity
}

John W. Goodrich

Lewis Research Center

Cleveland, Ohio

Karl Gustafson

University of Colorado

Boulder, Colorado

and Institute for Computational Mechanics in Propulsion

Lewis Research Center

Cleveland, Ohio

and

Kadosa Halasi

Kansas State University

Manhattan, Kansas

October 1989
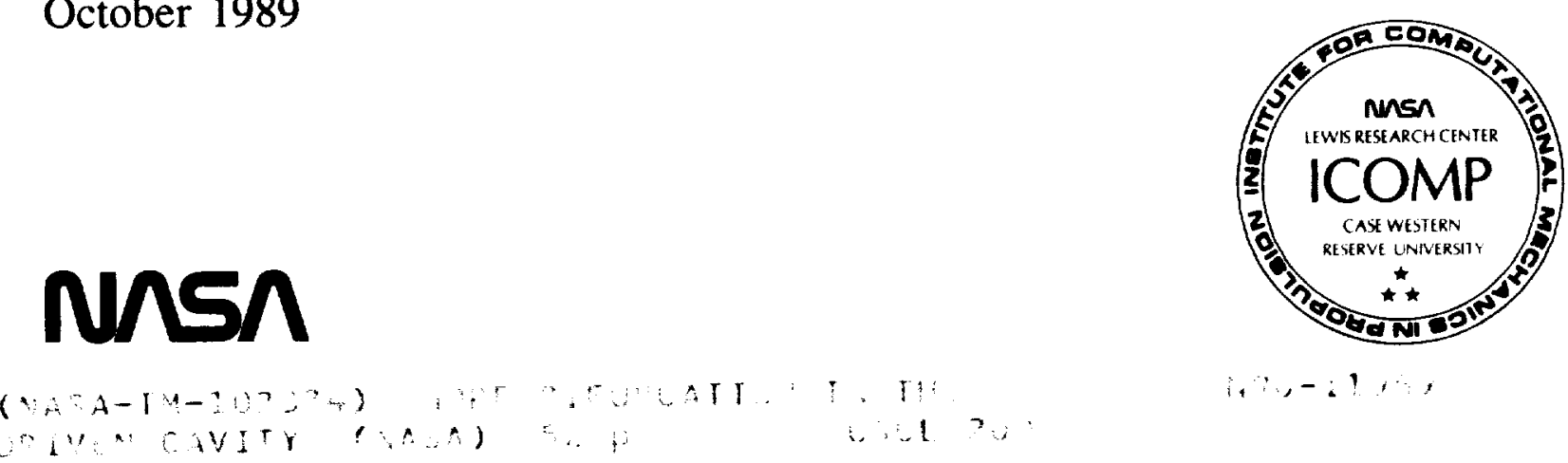

Hopf Bifurcation in the Driven Cavity

\author{
John W. Goodrich \\ National Aeronautics and Space Administration \\ Lewis Research Center \\ Cleveland, Ohio \\ Karl Gustafson \\ Department of Mathematics \\ University of Colorado \\ Boulder, Colorado \\ and Institute for Computational Mechanics in Propulsion* \\ Lewis Research Center \\ Cleveland, Ohio \\ and \\ Kadosa Halasi \\ Department of Mathematics \\ Kansas State University \\ Manhattan, Kansas
}

Summary

Incompressible two dimensional calculations are reported for the impulsively started lid driven cavity with aspect ratio two. The algorithm is based on the time dependent streamfunction equation, with a Crank-Nicolson differencing scheme for the diffusion terms, and with an Adams-Bashforth scheme for the convection terms. A multigrid method is used to solve the linear implicit equations at each time step. Periodic asymptotic solutions have been found for $R e=10000$ and for $R e=5000$. The $R e=5000$ results are validated by grid refinement calculations. The solutions are shown to be precisely periodic, and care is taken to demonstrate that asymptotic states have been reached. A discussion is included about the indicators that are used to show that an asymptotic state has been reached, and to show that the asymptotic state is indeed periodic.

*Work funded under Space Act Agreement C99066G. 


\section{1: Introduction}

Cavity flows have been a subject of study for some time. These flows have been widely used as test cases for validating incompressible fluid dynamics algorithms. The preponderance of such studies have addressed the steady flow problem, such as Ghia et. al. [4] and Schrieber and Keller [17], and the potentially rich unsteady cavity dynamics have only recently begun to be addressed. Greatly increased computational capabilities make possible the study of unsteady flows and initiates a new chapter in numerical analysis, determining the qualitative properties of the solutions of time dependent partial differential equations from their simulation. Examples of this type include a study of transition to turbulence in a two dimensional cascade flow by Fortin et. al. [3], and a study of the transition to chaos for a Boussinesq fluid in a vertical cavity by Paolucci and Chenoweth [16]. Recent work on the numerical analysis of large time and asymptotic solutions of partial differential equations includes the study of the convergence of attractors for finite dimensional approximate systems to the attractor of the original system by Hale et. al. [11], the study of the large time behavior of Galerkin approximations to Navier-Stokes equations by Constantin et. al. [2], and the study of finite element approximations of the nonstationary Navier-Stokes equations by Heywood and Rannacher [12]-[14], and especially for behavior as $t \rightarrow \infty$ in [14]. A theoretical treatment of the dynamical systems approach to the Navier-Stokes equations can be seen in Constantin and Foias [1], and in Temam [19]. Both the qualitative nature of asymptotic states, and the way in which they develop are new and exciting areas of research in partial differential equations. The driven cavity problem gives a computationally reasonable model fluid problem in which to investigate the qualitative features of the solution space of a physically reasonable infinite dimensional dissipative dynamical system. In contrast with other unsteady problems that are currently being investigated, the driven cavity is a self contained system with realistic nonperiodic boundary conditions, with steady nonperiodic forcing, and without artifical throughflow boundary conditions.

The vortex dynamics for unsteady Navier-Stokes flow in the driven cavity for various aspect ratios and Reynolds numbers with an impulsively started steady lid were studied in Gustafson and Halasi [8] and [9], and in Goodrich and Soh [7]. A persistant final oscillation 
in the aspect ratio two driven cavity with a $40 \times 80 \mathrm{grid}$ was shown in [9] at $R e=10000$ and $t \approx 300$, and the conjecture was raised that a qualitative transition had occurred to an unsteady asymptotic flow. The present paper continues that study by addressing the question of whether an unsteady asymptotic solution exists in the driven cavity with aspect ratio two. In section 2 we discuss some indicators for monitoring the development of a flow, for identifying an asymptotic flow state, and for presenting the qualitative nature of that state. In section 3 we present and discuss the algorithm that is used for the computations reported in this paper. In section 4 we discuss a periodic solution at $R e=10000$ on a coarse $48 \times 96$ mesh, comparing with and extending the results in [9]. In section 5 we present and discuss the main results of this paper, two periodic solutions at $R e=5000$ on $48 \times 96$ and $96 \times 192$ grids, with periods of approximately 2.477 and 2.309 , respectively. The two asymptotic states computed at $R e=\mathbf{5 0 0 0}$ have qualitatively similar dynamics. All three of the computed asymptotic states reported in this paper are periodic with very precise repetition of the asymptotic cycles. Based upon these computations we conclude that a Hopf bifurcation does occur in the aspect ratio two driven cavity for a critical Reynolds number $R e_{\mathrm{c}} \leq 5000$. In section 6 we discuss a number of continuing and remaining issues. 


\section{2: Measures of Qualitative Flow Features}

Flow visualization is a fundamental problem for computational fluid dynamicists as well as for experimentalists. This is especially true for the resolution of small scale flow details. A primary issue for the simulation of flow evolution to an asymptotic state is to identify measures that will give reasonable assurance that an asymptotic state has in fact been attained. This issue is somewhat obscured when investigating flows that converge to a steady state. We will list and briefly discuss the measures that we have used for the qualtitative representation of flow simulations.

\section{1: Field Representations}

The first series of measures and indicators represent the standard two dimensional flow field data. The representations that we have found useful are:

1) Streamfunction Contour Plots - These are useful for defining the large scale flow features. When overlayed with normalized velocity vectors, they give a good balance between large and small scale resolution.

2) Streamfunction Surface Plots - These plots are good for presenting large scale flow features. Like all surface plots they give an immediate and vivid impression of scaling throughout the flow field, but depending upon the view of the surface, prominant flow features can mask smaller scale structures.

3] Velocity Vector Plot - These plots are standard and are almost indispensible for actually visualizing the flow. Normalized velocity vectors are better for revealing the smaller scale structures.

4] Kinetic Energy Contour Plot - These show the momentum scale for the entire flow.

5] Kinetic Energy Surface Plot - These are extremely useful for showing the momentum scale for the flow and its various features. This is an excellant complement to either a normalized velocity vector plot or a streamfunction contour plot overlayed with normalized velocity vectors.

6] Vorticity Contour Plot - These are extremely useful for following vortex dynamics, particularly for vortex pairing and splitting as in [10]. Vorticity can be concentrated, so vorticity plots often reveal less than streamfunction contours about overall flow 
dynamics. Vorticity data is very useful for highlighting the resolution failures of an algorithm.

7] Vorticity Surface Plot - Since vorticity contours tend to be concentrated, a surface plot can give a better global view of the vorticity for a flow. Large regions of constant vorticity contrast well with local singularities and steep gradients.

8] Pressure Gradient Vector Plot - These clearly reveal principle vortex centers and their relative significance as field sources compared to secondary vortices.

The algorithm that we use for the results reported in this paper does not require a pressure solution, so we will not present any pressure data. The pressure gradient has been used to good effect by Gustafson and Halasi in [8]. All of the field representations that do not use pressure data were produced for this study, but for the sake of brevity the flow field will be represented just by streamfunction contour plots with and without normalized velocity vector overlays, and by surface plots of streamfunction, kinetic energy, and vorticity.

\section{2: Indicators of Dynamics}

The second series of measures and indicators are for tracking convergence to an asymptotic state, and for understanding the qualitative nature of that state. These various measures and indicators are scalars that are either global indicators or point data values. Global indicators can be obtained by using various mathematical norms on the flow data, or on the flow change data. Any of the scalar valued data can be obtained at each time step, and there are a variety of ways to display the information content of such scalar time series data. A simple graph of data plotted against time gives a quick and familiar view of the time changes in scalar data, although this type of presentation can mask important details. A spectral density or power spectrum is a well known device from signal processing which identifies the flow frequencies and their relative strengths. A phase diagram can be presented for scalar data either for one variable with the value at time $t_{n}$ plotted against the value lagged by $\mathrm{k}$ time iterations at $t_{n-k}$, or for two separate variables at the same time step plotted against each other. Spectral densities and phase diagrams are widely used to investigate and present the qualitative features of nonlinear dynamical systems. Both of these methods are particularly useful for classifying unsteady flows as either periodic 
or aperiodic, for identifying critical parameter values for transition between qualitatively different flows, and for identifying chaotic or turbulent flows. The indicators that we have found useful are:

9] The Relative $L_{1}$ Norm of the Streamfunction Change Per Time Step - This is a global measure, and is calculated as

$$
\frac{\sum_{i, j}\left|\psi_{i, j}^{n+1}-\psi_{i, j}^{n}\right|}{\sum_{i, j}\left|\psi_{i, j}^{n+1}\right|}
$$

This measure is similar to standard convergence indicators, and is easy to compute, but it is an integrated measure and tends to mask relatively small scale flow features. We did not look at the combined relative change in streamfunction and vorticity, which could be an interesting and more sensitive measure than just the streamfunction or vorticity data considered separately.

10] The Maximum and Minimum Streamfunction Value - These measures provide useful information about the location of the two main vortices in the cavity, and about the dynamics of the asymptotic solution at the center of each of these vortices. In addition to the global extremes of the streamfunction, it would be interesting to also record other local extremes in order to identify and track vortex centers, and to give their relative intensities.

11] The Relative $L_{1}$ Norm of the Vector Field Change Per Time Step - This is also a global convergence measure, and is calculated as

$$
\frac{\sum_{i, j}\left(\left|u_{i, j}^{n+1}-u_{i, j}^{n}\right|+\left|v_{i, j}^{n+1}-v_{i, j}^{n}\right|\right)}{\sum_{i, j}\left(\left|u_{i, j}^{n+1}\right|+\left|v_{i, j}^{n+1}\right|\right)} .
$$

We used this measure along with the relative $L_{1}$ norm of the streamfunction change data, and found that they behaved very similarly to each other. A choice between these two measures can be easily made on the basis of which variable set is being used.

12] The Total Kinetic Energy - Calculated for the entire grid minus the boundaries at each time step as

$$
\frac{1}{2} \Delta x \Delta y \sum_{i, j}\left\|u_{i, j}^{n+1}\right\|_{2}^{2}
$$


This measure was more sensitive than the streamfunction change data and was a very useful complement to that data. The time dependent dynamics of a flow are formulated in terms of momentum balance equations, so that such a global momentum measure would seem to be very appropriate data for the assessment of the evolution of a flow. In fact, our experience (see section 5) has indicated that very significant evolution can still occur even though the streamfunction or velocity time step change data becomes as small as $O\left(10^{-7}\right)$ or $O\left(10^{-8}\right)$, with the total kinetic energy remaining noticably sensitive to the continuing evolution of the flow. This experience suggests that common practices for stopping calculations could produce misleading results about the qualitative features of asymptotic flows.

13] The Maximum Acceleration - Determined for the entire interior grid as

$$
\max \left\{\frac{\left\|\mathbf{u}_{i, j}^{n+1}-\mathbf{u}_{i, j}^{n}\right\|_{2}}{\Delta t}\right\}
$$

The point where the maximum acceleration occurs can change with the time step, possibly in a very discountinuous way. Consequently, this indicator can be sensitive to the time scales and dynamics of both the local change per time step in the point values of the velocity field, and the global convective transport processes within the flow. There can be subtle and complex relationships between these processes. Note that this indicator is not the maximum convective derivative, which would be easy to obtain and could be interesting to try.

14] Streamfunction Value at a Point

15] Velocity Component at a Point

16] Kinetic Energy Value at a Point

17] Vorticity Value at a Point

For the calculations reported in this paper we found the relative $L_{1}$ streamfunction change norm and the total kinetic energy to be very good complements for indicating convergence to an asymptotic state. The streamfunction change per time step data showed convergence earlier, and the total kinetic energy showed final convergence to the asymptotic state. This conclusion was augmented by point data time series for streamfunction, kinetic energy, and 
vorticity. The sensitivity of the indicators increased from the streamfunction, to the velocity components defined as streamfunction derivatives, then to the kinetic energy defined with squared velocity components, and finally to the vorticity defined as the Laplacian of the streamfunction. 
3: An Algorithm For Time Dependent Incompressible Navier-Stokes Equations

This section briefly presents the implicit finite difference streamfunction algorithm for the unsteady incompressible Navier-Stokes computations reported this paper. Further specific algorithm details may be found in Goodrich [6] or Goodrich and Soh [7]. An explicit MAC (marker and cell) primitive variable scheme was used in [8] and [9]. The time step restriction for such an explicit scheme is prohibitive for truly long time studies. The qualitative flow dynamics of both codes agree for the computations that are comparable.

\section{1: Flow Equations}

In a bounded open region $\Omega$, the general dimensionless Navier-Stokes equations for incompressible flows are

$$
\begin{aligned}
\frac{\partial \mathbf{u}}{\partial t}+(\mathbf{u} \cdot \nabla) \mathbf{u}-\frac{1}{R e} \Delta \mathbf{u}=-\nabla p+\mathbf{F}, \text { for } \mathbf{x} \text { in } \Omega, \text { and } t>0, \\
\nabla \cdot \mathbf{u}=0, \text { for } \mathbf{x} \text { in } \Omega, \text { and } t>0, \\
\mathbf{u}(\mathbf{x}, 0)=\mathbf{a}(\mathbf{x}), \text { for } \mathbf{x} \text { in } \Omega, \text { at } t=0, \\
B[\mathbf{u}(\mathbf{x}, t)]=\mathbf{b}(\mathbf{x}, t), \text { for } \mathbf{x} \text { in } \partial \Omega, \text { and } t>0,
\end{aligned}
$$

where $u$ is the velocity, $p$ is the scaled pressure, $R e$ is the Reynolds number, $F$ is the volume force per unit mass, $\partial \Omega$ is the boundary of $\Omega$, and $B$ is the operator that defines the boundary conditions. We will ignore the body force $F$. For the simulations of two dimensional cavity flows that we will be reporting, the computational domain $\Omega$ is just a rectangle as in Figure 1. The length and velocity scales for these cavity flows are taken to be the lid length and velocity, so that the lid velocity is always 1 . The velocity field for two dimensional flows may be written in terms of the streamfunction $\psi$ as

$$
u(x, t)=\frac{\partial \psi}{\partial y} \text { and } v(x, t)=-\frac{\partial \psi}{\partial x}, \text { for } \mathbf{x} \text { in } \Omega \text { and } t \geq 0,
$$

and the dimensionless equations for evolution of time dependent viscous incompressible flows may be written as the streamfunction equation

$$
\frac{\partial \Delta \psi}{\partial t}=\frac{1}{R e} \Delta^{2} \psi+\frac{\partial \psi}{\partial x} \Delta \frac{\partial \psi}{\partial y}-\frac{\partial \psi}{\partial y} \Delta \frac{\partial \psi}{\partial x}, \text { for } \mathbf{x} \text { in } \Omega, \text { and } t>0
$$


In this formulation the data for the impulsively started driven cavity consists of the initial values

$$
\psi(x, 0)=0, \text { for } x \text { in } \Omega, \text { at } t=0
$$

and the standard boundary conditions (see Figure 1)

$$
\psi(x, t)=0, \text { for } x \text { in } \partial \Omega, \text { and } t>0
$$

$$
\begin{aligned}
& \frac{\partial \psi}{\partial \eta}(x, t)=1, \text { for } x \text { on the cavity lid, and } t>0 \\
& \frac{\partial \psi}{\partial \eta}(x, t)=0, \text { for } x \text { on the cavity walls, and } t>0
\end{aligned}
$$

where $\frac{\theta}{\partial \eta}$ is differentiation in the exterior normal direction at the boundary.

\section{2: The Discretization}

The discrete approximation for the streamfunction $\psi=\{\psi(\mathbf{x}, t): \mathbf{x} \in \Omega, t>0\}$ will be taken to be $\tilde{\mathbf{z}}=\left\{\tilde{\mathbf{z}}^{m}: m=0,1, \ldots\right\}=\left\{z_{i, j}^{m}: i=0,1, \ldots I, j=0,1, \ldots J, m=\right.$ $0,1, \ldots\}$ on the rectangular computational mesh. We will use a uniform grid on the rectangular domain, and we will use centered spacial differencing for equation (1b), with a Crank-Nicolson time differencing for the diffusion terms, and a second order AdamsBashforth time differencing for the convection terms. Let La be the conventional five point centered difference approximation to the Laplacian, let $\mathrm{Bi}$ be the conventional thirteen point centered difference approximation to the Biharmonic operator, and let $\delta_{x}$ and $\delta_{y}$ be the conventional centered difference operators

$$
\delta_{x}\left(\tilde{z}^{m}\right)_{i, j}=\frac{z_{i+1, j}^{m}-z_{i-1, j}^{m}}{2 \Delta x}, \text { and } \delta_{y}\left(\tilde{z}^{m}\right)_{i, j}=\frac{z_{i, j+1}^{m}-z_{i, j-1}^{m}}{2 \triangle y}
$$

With this notation and differencing scheme, we may discretize equation (1b) as

$$
\begin{aligned}
& \mathrm{La}\left(\tilde{\mathrm{z}}^{n+1}\right)-\frac{\Delta t}{2 \operatorname{Re}} \mathrm{Bi}\left(\tilde{\mathrm{z}}^{n+1}\right) \\
&=\mathrm{La}\left(\tilde{z}^{n}\right)+\frac{\Delta t}{2 \operatorname{Re}} \operatorname{Bi}\left(\tilde{z}^{n}\right)-\frac{3 \Delta t}{2}\left[\delta_{x}\left(\delta_{y}\left(\tilde{z}^{n}\right) \mathrm{La}\left(\tilde{z}^{n}\right)\right)-\delta_{y}\left(\delta_{x}\left(\tilde{z}^{n}\right) \mathrm{La}\left(\tilde{z}^{n}\right)\right)\right] \\
&+\frac{\Delta t}{2}\left[\delta_{x}\left(\delta_{y}\left(\tilde{z}^{n-1}\right) \operatorname{La}\left(\tilde{z}^{n-1}\right)\right)-\delta_{y}\left(\delta_{x}\left(\tilde{z}^{n-1}\right) \mathrm{La}\left(\tilde{z}^{n-1}\right)\right)\right] .
\end{aligned}
$$


In order to incorporate the boundary condition (1e), the standard finite difference approximations are used for modifying the discrete equation (2) at grid points next to the boundary. Note that this algorithm is second order accurate in both time and space. The velocity components $\left(u_{i, j}^{m}, v_{i, j}^{m}\right)$ are directly recovered from the discrete streamfunction solution as

$$
u_{i, j}^{m}=\frac{1}{2 \Delta y}\left(z_{i, j+1}^{m}-z_{i, j-1}^{m}\right), \text { and, } v_{i, j}^{m}=-\frac{1}{2 \Delta x}\left(z_{i+1, j}^{m}-z_{i-1, j}^{m}\right) .
$$

Notice that the velocity components are both defined at each grid point and not at different locations on a staggered grid. In our formulation we use a grid centered central difference expression for the mass conservation equation. The velocity solution is exactly discretely divergence free with respect to this divergence operator.

\section{3: A Solver}

The operators in the implicit time stepping equation (2) do not change with time. In fact, the discrete streamfunction solution $\tilde{\mathbf{z}}^{n+1}$ from equation (2) depends upon the time step only through the solutions at the previous two time steps. The problem that must be solved at each time step may be written as

$$
\operatorname{La}\left(\tilde{\mathbf{z}}^{n+1}\right)-\frac{\Delta t}{2 \operatorname{Re}} \operatorname{Bi}\left(\tilde{\mathbf{z}}^{n+1}\right)=\tilde{\mathbf{f}}\left(\tilde{\mathbf{z}}^{n}, \tilde{\mathbf{z}}^{n-1}\right)
$$

where $\tilde{f}$ is the discrete source term from the right hand side of (2). This problem is a discretization of

$$
\Delta \psi-\frac{\Delta t}{2 R e} \Delta^{2} \psi=\mathbf{f}, \text { for } \mathbf{x} \text { in } \Omega, \text { at } t=t_{n+1},
$$

an elliptic partial differential equation that combines the Laplace and Biharmonic operators. At each time step we use a multigrid method to solve these equations, as in Goodrich [6]. The Biharmonic operator is factored as two Laplacians as in Linden [15], point GaussSeidel relaxation is used for the smoothing operator, and linear restriction and prolongation operators are used. A V-cycle iteration scheme is used, with 3 iterations per grid level while coarsening, and none while refining. At each time step, 10 to 15 iteration cycles are used to reduce the residuals in (3) to less than $5.0 \times 10^{-12}$. 


\section{4: Numerical Results At $R e=10000$}

The first numerical results that suggest a periodic solution for the driven cavity with aspect ratio two are in Gustafson and Halasi [9]. These computations are at $R e=10000$ for $0 \leq t \leq 360$, using an explicit primitive variable Euler-MAC scheme on a $40 \times 80$ grid with $\Delta t=\frac{1}{1000}$. The published results are a series of normalized velocity vector plots of the flow field that suggest an eventual periodic pattern of evolution and interaction in the cavity vortex dynamics. The grid resolution that was used at this Reynolds number leaves open the possibility that the computational mesh might be filtering out dynamical effects with smaller spatial scales. The reliance upon just the normalized vector plots leaves open the question of whether a periodic, a quasi-periodic, or an aperiodic flow was actually observed. The relatively short time interval that was calculated and the small number of flow periods that were observed does not ensure that the initial transients were fully dissipated and that a true asymptotic solution was obtained.

An independent series of calculations was initiated to verify the results reported by Gustafson and Halasi [9]. These computations are at $R e=10000$ for $0 \leq t \leq 1800$, using the algorithm described in section 2 on a $48 \times 96 \mathrm{grid}$ with $\Delta t=\frac{1}{64}$. These computations show that the asymptotic state on this grid is a periodic flow, although the asymptotic state occurs only at a much later time than suggested by Gustafson and Halasi [9]. The results of these computations are similar to the data for the stronger results that we have obtained at $R_{e}=5000$, which are the focus of this paper, so for the sake of brevity the data at $R e=10000$ will be discussed but will not be shown. Several indicators were used to ensure that an asymptotic state was actually reached. The first global indicator is the relative $L_{1}$ norm of the streamfunction change per time step. This indicator suggests that a periodic asymptotic state has been reached at $t \approx 300$, as reported in Gustafson and Halasi [9]. The global maximum and minimum streamfunction values were tracked as the second and third global indicators of approach to an asymptotic state. The observed data showed that as the flow evolved, the maximum and minimum streamfunction values on the grid begin to oscillate at the centers of the upper and lower vortices. The maximum streamfunction value in the secondary lower vortex reaches its asymptotic oscillation at $t \approx 550$, and the minimum streamfunction value in the primary upper vortex reaches its asymptotic 
oscillation at $t \approx 900$. A fourth global indicator is the relative $L_{1}$ norm of the vector field change per time step. This indicator suggested that a periodic asymptotic state had been reached at $t \approx 500$. The fifth global indicator is the total kinetic energy for the entire grid minus the lid. This data reaches a periodic asymptotic state at $t \approx 1500$. Besides this global data, local flow data was gathered at selected fixed points in the grid. At points with coordinates $(i, j)=(44,50)$ and $(i, j)=(16,46)$ the values of the streamfunction, the $x$ and $y$ velocity components, and the vorticity were recorded, and the kinetic energy at the point was calculated, at each time step for $1600 \leq t \leq 1800$. The first point is near the end of the wall jet that descends from the lid along the upper half of the downstream wall. The second point is near the end of the bounded shear layer as it approaches the wall after crossing the middle strip of the cavity. This data shows clean spectral signatures with one fundamental frequency. The data at the end of the bounded shear layer shows energization of harmonics of the one fundamental frequency as a result of convection through the shear layer. Based upon all of these indicators the discrete dynamics on this grid do appear to reach a periodic asymptotic state at $t \approx 1500$, with a period between 223 and 224 time steps, or between 3.48 and 3.50 nondimensional time units. The periodic asymptotic flow on this $48 \times 96$ grid at $R e=10000$ is qualitatively the same as the vortex dynamics observed in [9].

A natural question is whether or not the periodic flow for this discrete system is a grid dependent phenomenon. This is a particularly relevant question for this simulation since the best steady solutions for the square cavity at $R e=10000$ are on a $256 \times 256$ uniform grid, and the $48 \times 96$ uniform grid that we are using clearly does not give anywhere near the same resolution. Another serious cause for concern is that the results reported by Gustafson and Halasi [9] on a $40 \times 80$ grid suggested a period of approximately 4.5 , while our $48 \times 96$ grid calculation gives a period between approximately 3.48 and 3.50 . To begin checking for grid dependence we conducted a calculation at $R e=10000$ for $0 \leq t \leq 500$ using the algorithm in section 3 on a $96 \times 192$ grid with $\Delta t=\frac{1}{128}$. This calculation shows a much more complex time evolution than the results for the same time interval on the $48 \times 96 \mathrm{grid}$, and it is possible that the $96 \times 192 \mathrm{grid}$ simulation is itself masking even finer scale dynamics. This suggests that the coarser grid was filtering smaller scale 
dynamic processes that could contribute to a more complex asymptotic state than a single frequency periodic flow. We have in fact concluded that the $R e=10000$ simulation on a $48 \times 96$ grid is most likely inadequate for resolving all but the coarsest features of the cavity flow, and the periodic discrete solution on the coarse grid at $R e=10000$ cannot be accepted as giving a reliable portrait of the actual asymptotic continuum flow dynamics. Consequently, we discontinued the calculations at $R e=10000$ and decided to initiate discrete simulations at $\boldsymbol{R e}=\mathbf{5 0 0 0}$ where less grid resolution would be required to capture the continuum dynamics. 


\section{5: Numerical Results At $R e=5000$}

Two different simulations were computed to asymptotic periodic states at $R e=5000$ for the impulsively started driven cavity with aspect ratio two. Both simulations use the algorithm described in section 3. The first simulation uses a $48 \times 96$ grid with $\Delta t=\frac{1}{64}$, and the second simulation uses a $96 \times 192$ grid with $\Delta t=\frac{1}{128}$. The simulations at $R e=10000$ and $R e=5000$ are similar in the sense that they all demonstrate a very clean and qualitatively similar periodic solution to the nonlinear discrete dynamical system that is being used as an approximation to the fluid flow in the cavity. The conclusion of the discussion in Section 4 about the $R e=10000$ simulations is that a $48 \times 96$ grid is inadequate for resolving all but the coarsest features of the cavity flow, so the calculated periodic discrete solution on the coarse grid at $R e=10000$ cannot be accepted as giving a reliable portrait of the actual asymptotic continuum flow dynamics. The $R e=5000$ simulations are essentially different in this regard, and we may reasonably conclude that they are likely to represent at least the qualitative dynamic features of the actual continuum flow dynamics. The first supporting evidence is that the refined $96 \times 192$ grid has a resolution close to the $128 \times 128 \mathrm{grid}$ used in Goodrich [6] with the same algorithm to produce a steady square cavity solution in good agreement with the standard published $R e=5000$ solutions. The second supporting evidence is that the grid refinement calculation produces flow dynamics that are close to the coarse grid results. In particular, the period given by the coarse grid simulation is $2.469 \leq T \leq 2.484$, while the refined grid simulation gives $2.305 \leq T \leq 2.313$.

\section{1: $R e=5000$ on a $48 \times 96$ Grid}

The computation at $R e=5000$ on the coarser $48 \times 96$ grid will be presented with only a small amount of detail. The computation is for $0 \leq t \leq 4100$, and the flow reaches a periodic asymptotic state at $t \approx 1100$ with a period between $\frac{158}{64} \approx 2.469$ and $\frac{159}{64} \approx 2.484$. This flow is completely periodic with no apparent secular trend for $1100 \leq t \leq 4100$, or for approximately 1200 oscillation cycles.

The entire flow field at $t=4000$ is shown in Figure 2 as a streamfunction contour plot. The main features of the flow are the primary and secondary circulations in the 
upper and lower cavity, the wall jet that descends from the lid along the upper half of the downstream wall, and the wavey shear layer between these two main circulations. There are tertiary corner vortices in the two lower corners that are separated from each other by one sixth of the bottom wall where the secondary vortex in the lower cavity is attached to the lower boundary. Surface plots of streamfunction, kinetic energy, and vorticity show some lack of grid resolution especially in the boundaries near the end of the wall jet, and in the upper left corner. The flow at this instant is generally representative of the flow at any time during the entire cycle of the periodic asymptotic state. The vortex dynamics for this periodic asymptotic solution are similar to the dynamics for the refined $96 \times 192$ grid. A key feature of the periodic vortex dynamics is the appearance of a pair of small counterrotating tertiary vortices along the downstream wall slightly below the end of the wall jet descending from the lid. The stronger and higher tertiary vortex periodically appears below the wall jet and is convected as a wavy disturbance along the shear layer between the primary and secondary vortices in the cavity. More detail will be presented for the similar dynamics of the refined grid solution.

Figure 3a shows the spectral signature of the streamfunction at the point with coordinates $(i, j)=(44,50)$ or $(x, y) \approx(0.92,1.04)$, and Figure $3 b$ shows this data as a phase portrait plot of $\left(\psi_{i, j}^{n-15}, \psi_{i, j}^{n}\right)$, both for $4000 \leq t \leq 4100$. Similar spectral signatures and phase portraits are shown for the kinetic energy in Figures $3 c-d$, and for the vorticity in Figures 3e-f. This point is near the end of the wall jet descending from the lid along the downstream wall, and the data represents a sample with 6400 time points, with between 158 and 159 data points in each period, and for more than 40 periods. Figures $4 a-f$ show similar data recorded at a point near the end of the bounded shear layer across the middle of the cavity with coordinates $(i, j)=(16,46)$ or $(x, y) \approx(0.33,0.96)$. The values of streamfunction, kinetic energy, and vorticity from these two points all have extremely clean spectral signatures with one fundamental frequency. Harmonics are energized especially for vorticity by the effect of the bounded shear layer. The narrowness of the plot lines indicates the precise repetitiveness of this data for these $40^{+}$periods. This point data indicates a precisely periodic solution at $R e=5000$ on the $48 \times 96$ grid. 


\section{2: $R e=5000$ on a $96 \times 192$ Grid}

The computation at $R e=5000$ with the finer $96 \times 192$ grid is the main result and will be presented in some detail. The computation was for $0 \leq t \leq 4100$, and the flow reaches a periodic asymptotic state at $t \approx 3700$ with a period between $\frac{295}{128} \approx 2.305$ and $\frac{296}{128} \approx 2.313$. This flow is completely periodic with no secular trend for $3700 \leq t \leq 4100$, or for more than 170 oscillation cycles.

A plot of the relative $L_{1}$ norm of the streamfunction change per time step is shown for $5 \leq t \leq 100$ in Figure 5a, for $100 \leq t \leq 4000$ by intervals of 300 nondimensional time units in Figures 5b-n, and for $4000 \leq t \leq \mathbf{4 1 0 0}$ in Figure 5o. The total kinetic energy is shown for $5 \leq t \leq 100$ in Figure $6 \mathrm{a}$, shown by intervals of 300 nondimensional time units for $100 \leq t \leq 4000$ in Figures $6 b-n$, and for $4000 \leq t \leq 4100$ in Figure 6o. Note that the vertical scales in Figures 5 and 6 are all different, and that there are two time scales in each figure. The data in Figures 5 and 6 shows a dramatic history. Up to $t \approx 700$ the time history seems to be following a dissipating evolution. At $t \approx 700$ the relative $L_{1}$ change per time step and the total kinetic energy both start to increase in absolute level with oscillations that increase in amplitude. There is also a beating process with the interplay of multiple frequencies. There is a peak in the global kinetic energy at $t \approx 1600$, and then the global kinetic energy decreases until $t \approx 2600$, with continuing increases in the amplitude of the kinetic energy oscillations. At $t \approx 2600$ the streamfunction time step change starts to decrease while the kinetic energy begins to increase again. Between $t=2700$ and $t=2800$ both variables start to oscillate in what seems to be an erratic manner until $t \approx 2950$. After this the data steadies down and becomes very regular until the streamfunction time step change data converges at $t \approx 3250$, and the kinetic energy data converges at $t \approx 3700$. Contour and surface plots for streamfunction, kinetic energy, and vorticity on the refined grid show no visually apparent changes for $500 \leq t \leq 2000$. Noticable pulsating changes begin to occur in this surface data only for $2000 \leq t \leq 2500$, along with a noticable increase in the momentum in the lower half of the cavity. Note that the relative $L_{1}$ norm of the streamfunction change per time step is $O\left(5 \times 10^{-7}\right)$ for $t \approx 700$. This is below what might easily be taken as a small test value used to stop the calculation with the result declared to be a steady asymptotic state. The actual asymptotic state has 
extremely regular oscillations in the relative $L_{1}$ norm of the streamfunction change per time step, with relative $L_{1}$ norm values that are $O\left(3 \times 10^{-1}\right)$ for $t \geq 3700$.

The precisely periodic nature of the asymptotic flow is shown in Figure $7 \mathrm{a}$ by the spectral density of the relative $L_{1}$ norm of the streamfunction change per time step, and in Figure 7b by the spectral density of the total kinetic energy, both for $4000<t \leq 4100$. Both spectral signatures show a single fundamental frequency near $0.433 \approx \frac{1}{2.31}$. Note that the streamfunction change data has one harmonic. Both of these spectral signatures are for global data, and reflect the global dynamics on the entire grid. The global maximum and minimum streamfunction values were also recorded, and are shown together in Figure 7c as a phase portrait plotting $\left(\psi_{\max }^{n}, \psi_{\min }^{n}\right)$ for $4000<t \leq 4100$. This plot is for data taken at 12800 discrete time steps, with between 295 and 296 time steps per cycle, for more than 43 complete cycles. All of this data is plotted sequentially with time, so that the physical plot lines have been drawn over 43 times. The narrowness of the lines substantiates the precisely periodic nature of the asymptotic solution. The global minimum and maximum always occured at the centers of the primary and secondary vortices in the upper and lower halves of the cavity. The effect of the periodic asymptotic flow is still apparent even in the centers of the two largest vortices. The entire streamfunction surface is vibrating with extreme precision. Note that the two extremes are oscillating approximately one half of a period out of phase with each other.

A qualitative portrait of the overall solution field at the single instant $t=4100$ is given in Figures 8a-d. The streamfunction surface plot in Figure 8a shows a wavey disturbance or swelling that is periodically convected by the bounded shear layer across the middle of the cavity. This particular view shows the disturbance just before it is absorbed and dissipated by the boundary layer flow at the end of the bounded shear layer. The kinetic energy surface plot in Figure $8 \mathrm{~b}$ shows a periodic wave in the momentum ridge near the center foreground. The dramatic low point in the kinetic energy ridge is in the bounded shear layer just above the front of the streamfunction swelling, with local maxima and minima in the kinetic energy just upstream from this low point. There seems to be a periodic pulse of momentum associated with the periodic streamfunction disturbance. The vorticity surface is shown in Figure 8c. Note that the surface plots of 
streamfunction, kinetic energy, and vorticity all show smooth resolution of the flow solution over the entire grid. The streamfunction contour plot in Figure $8 \mathrm{~d}$ shows a recirculating boundary layer at the bottom of the cavity. This feature appears in the evolution of the flow before $t=500$. Note that the streamline defining this feature is for $\psi=-0.001$, while the streamline closest to the center of the primary circulation is for $\psi=-0.090$. This recirculating boundary layer in the bottom of the cavity is imperceptible in the surface plots of streamfunction, kinetic energy, and vorticity, but it may be highlighted by a low enough streamfunction contour or a normalized velocity vector plot. There are two local weak tertiary recirculations diagonally out of the corners, within the ends of the figure eight shaped streamline for $\psi=-0.090$, and above the recirculating boundary layer along the lower wall. It appears that a single large third vortex is partially formed in the bottom of the cavity, but that there is not quite enough room below the secondary vortex to allow for the joining of the two weak tertiary vortices above the lower wall. The refined grid calculation shows quartiary corner vortices, resolved with between one and four grid points. The recirculating boundary layer along the lower wall is not present in the coarse grid asymptotic flow, which has two tertiary vortices in the lower corners separated by a short stretch of grid points where the secondary vortex attaches to the lower wall. The asymptotic coarse grid solution has a smaller total amount of kinetic energy on the interior grid than the asymptotic flow on the refined grid. These differences between the two asymptotic flow solutions are probably caused by the better resolution of gradients and momentum diffusion on the refined grid.

Figures 9a-k are a series of eleven plots at time intervals of 0.25 over slightly more than one complete periodic cycle starting at $t=4100$. These eleven plots are streamfunction contours with normalized velocity vector overlays in a central band across the cavity, and they show the essential vortex dynamics in detail for a typical cycle of the periodic asymptotic flow. The first interesting feature of these dynamics is the wall jet that descends from the lid along the upper half of the downstream wall, with the periodic appearance and interaction of two small counter rotating tertiary vortices near the tip of this wall jet below where it errupts into the flow field as a whole. Note that the secondary vortex in the lower half of the cavity creates a relatively weak flow that rises along the lower half of the 
downstream wall. The triggering instability that generates the asymptotic periodic flow is possibly the interaction of these two opposing flows. The relative strength of these two small tertiary vortices is related to the relative strength of the two opposing flows along the downstream wall. The second interesting feature of these dynamics is the evolution of the flow as the stronger upper small tertiary vortex is convected by the bounded shear layer across the middle of the cavity. The existence of local recirculation in the flow is possible because there are local streamfunction extremes in the center of the small vortices as they leave the wall, with saddle points in the streamfunction surface between the small vortices and the lower secondary vortex. The upper small tertiary vortex is convected to the streamfunction surface of the larger secondary vortex, the saddle point disappears, and the smaller vortex loses its independent identity to become a swelling on the side of the streamfunction surface of the larger vortex. This local swelling on the streamfunction surface is then convected across the bounded shear layer as a wavy disturbance in the streamfunction contour lines until the wall at the end of the shear layer is reached, where the local swelling is dissipated by the boundary layer. The bounded shear layer has an effect on the spectral signature of the local flow variables, since the nonlinear convection terms energize integer multiples of the basic frequency of the periodic shedding process at the end of the wall jet. In its final effects, the upper small vortex disturbs the flow all the way around the two large circulation patterns. Kinetic energy contours show this effect near the upper lid, and a close inspection of kinetic energy and vorticity surface plots shows an effect all along the upstream wall, and along both the lid and the lower wall. These effects away from the central band in the cavity are slight. The final interesting feature of these dynamics is the history of the lower small counterrotating tertiary vortex which originates a little later and slightly below the upper small vortex. This local flow pattern is convected away from the wall sooner than the upper small vortex, but it is weaker than the upper small vortex, and as the upper small vortex emerges into the flow the lower small vortex is pushed back toward the wall below its point of origin to be partially dissipated by the boundary layer.

Figures 10a-i shows nine plots of data at the particular point with indexes $(i, j)=$ $(88,120)$, or $(x, y) \approx(0.92,1.25)$, and for $4000<t \leq 4100$. This point is near the end of 
the wall jet that descends from the downstream end of the moving lid. The first three plots in Figures 10a-c are for the spectral densities of the point values of the streamfunction, the kinetic energy, and the vorticity. Figure $10 \mathrm{~d}$ is a phase portrait of $\left(\psi^{n-31}, \psi^{n}\right)$, or the streamfunction point values plotted with a lag of 31 time steps. Figures $10 \mathrm{e}$ and 10f are similar time lagged phase portraits for the point values of the kinetic energy and the vorticity. Figures 10g-i are phase portraits of $\left(\psi_{i, j}^{n}, \frac{1}{2}\left\{\left(u_{i, j}^{n}\right)^{2}+\left(v_{i, j}^{n}\right)^{2}\right\}\right),\left(\psi_{i, j}^{n}, \omega_{i, j}^{n}\right)$ where $\omega$ is the vorticity, and $\left(u_{i, j}^{n}, v_{i, j}^{n}\right)$, respectively. Figures 11a-i shows nine similar plots at the particular point with indexes $(i, j)=(32,106)$, or $(x, y) \approx(0.33,1.10)$, and for $4000<t \leq 4100$. This point is approximately one third of the distance from the end of the bounded shear layer as it approaches the wall opposite the wall jet after crossing the center of the cavity. The data in Figures 10 and 11 is for every time step with a sample of 12800 time points for $4000<t \leq 4100$, with 295 iterations per cycle, and with slightly more than 43 cycles during this time interval. All of this data is represented in each of these plots. Notice the appearance of multiples of the fundamental harmonic in the data from the point near the end of the bounded shear layer. The point data in these figures shows the precisely periodic nature of the asymptotic flow with a fundamental frequency of approximately $0.433 \approx \frac{1}{2.31}$.

It was quite surprising to have to run the finer grid flow so long in order to obtain an asymptotic periodic state, since the coarse grid simulation converged at $t \approx 1100$. The recirculating boundary layer along the lower wall is present only in the refined grid asymptotic flow. The refined grid asymptotic solution has a larger total amount of kinetic energy on the interior grid than the coarse grid asymptotic flow. These differences between the two asymptotic flow solutions could be caused by a better resolution of gradients and momentum diffusion on the refined grid, particularly in the boundary layers. The precise long term repetition of the periodic asymptotic state, the general qualitative agreement between the asymptotic dynamics of the coarse and fine grid solutions, and the general qualitative agreement between the two coarse grid simulations at $R e=10000$ with different algorithms, all lead us to believe that the computed solution is a genuine periodic asymptotic state and not just a numerical artifact. 


\section{6: Remarks and Discussion}

We would like to include some general remarks about numerical results and methodology as they pertain to this general new problem of computationally determining the qualitative nature of unsteady flows, especially for the long time behavior of unsteady flows.

\section{1: Methodology}

There are two views toward determining the qualitative behavior of a fluid flow. The first more predominant and easier view is to restrict attention to the branching diagram of the stationary steady flow problem. This is the bifurcation theory approach. The second view is to follow the unsteady flow to its final or asymptotic state. This is the dynamical systems approach. A main goal of both approaches is to determine all stable flow configurations. Both approaches may be said to be roughly equivalent if attention is restricted to stationary final states with $\mathbf{u}_{i}=0$. When progressing to the determination of qualitative flow behavior beyond the steady state the two approaches can yield different conclusions.

In order to determine the transition to a periodic solution as the result of a Hopf bifurcation, the bifurcation theory approach typically uses an extended set of steady equations by finding the eigenvalues of an associated Jacobian matrix, and then by finding the bifurcation parameter value at which a complex conjugate pair of such eigenvalues crosses the imaginary axis. Some arguments for the bifurcation theory steady equation analysis are: (1) computational cost is lower than an unsteady analysis; (2) the critical threshold parameter is predicted "exactly", although after several mesh refinements; (3) the variation of the threshold parameter with other parameters can be studied in a similar way.

Some arguments for using the dynamical systems approach of following an evolving flow to its asymptotic state are: (1) a time dependent computation "should" follow the "physics", so stable branches can be explored with continuation procedures; (2) more complicated asymptotic states that occur further along a bifurcation diagram than the initial bifurcation points can be explored; (3) the dependence of the final state on initial 
and intermediate conditions can be readily tested; (4) all of the intervening dynamics will be exhibited; (5) a complete picture of the final state is available within the limits of truncation error. We note that the unsteady dynamical systems approach as presently formulated does not readily pinpoint critical parameter threshold values for bifurcations.

\section{2: Solutions}

It is useful to draw some distinctions between various possible numerical flow solutions. A numerical algorithm for a fluid flow calculation is a system of algebraic equations, and this system of algebraic equations can have multiple solutions. Some of these solutions are valid and some are spurious. To quote Schreiber and Keller [18]:

“... Furthermore, since the nonlinearity in the Navier-Stokes equations is quadratic, the approximating algebraic equations are also quadratic (in any reasonable scheme). In the two-dimensional case with uniform mesh $h$ in a domain of diameter $O(1)$ there are essentially $N^{2}=\frac{1}{h^{2}}$ unknowns and coupled quadratic equations. Now a basic result in algebraic geometry assures us that this algebraic system has $2^{N^{2}}$ solutions, although some minor difficulties, i.e., "common intersection components," must be eliminated or else there can be manifolds of solutions. If the flow problem of interest has a unique solution, we must hope that one of these $2^{N^{2}}$ solutions is a close approximation to it and that all of the others is spurious. This cursory account suggests that most of the numerical solutions are spurious!

Fortunately most of the "numerical" solutions are also complex, so real computations do not usually reveal them. Furthermore, solution procedures using continuation from known physical states may avoid them. But this is not always the case as we show in this note. Indeed even time marching schemes may lead to spurious steady states. Our results have revealed that this is particularly so when upstream differencing has been used in the driven cavity problem.

Unfortunately there is at present no good theory to determine when a solution of the approximating problem is spurious and when it is "legitimate." Indeed this imposes a severe burden on the computational fluid dynamicist to make additional tests on his results which will add weight to his assertion of their legitimacy. These tests 
may affirm known physical or mathematical properties of the flow or else they may assure known approximation properties of the numerical method (i.e., $h^{2}$-truncation expansion, etc.)."

We have addressed this issue by checking that our code and unsteady simulation approach accurately reproduce the known steady state solutions for the aspect ratio one driven cavity at $R e=5000[6]$, and by checking that our simulations reproduce the same qualitative periodic flow dynamics at $R e=5000$ on both coarse and refined grids.

In a study of the aspect ratio one driven cavity, Glowinski, Keller, and Rinehart $[5, \mathrm{p} 831]$ have said:

"A most interesting question is the possible occurence of multiple solutions as the Reynolds number increases beyond some critical value. ... Actually and to our knowledge the computed solutions obtained in the range $0 \leq R e \leq 5000$ by various authors using different methods agree quite well; this observation suggests that multiple solutions can only appear for greater values of $R e . "$

The aspect ratio one driven cavity has corroborated solutions for $0 \leq R e \leq 10000$ using both steady and unsteady algorithms. These various solutions differ from each other in terms of resolution and vortex details, but they are all steady solutions, and qualitatively similar in terms of the large scale vortex structures.

An example of qualitative flow features that can distinguish solutions is given by Stokes flow with reasonable symmetry assumptions in lower cavity corners. A sequence of more than twenty corner vortices was resolved by Gustafson and Leben [10], where the smallest intensities were $O\left(10^{-80}\right)$. This type of detail in a steady flow solution points out that numerical solutions can be distinguished by resolution which may not have any dynamical significance. There is a dynamically significant difference between steady and periodic asymptotic flow solutions.

There are several known and mutually agreeing steady numerical solutions of the aspect ratio two driven cavity for $0 \leq R e \leq 2000$, plus the periodic solutions that we are reporting in this paper for $R e=5000$. One of the features distinguishing the coarse grid and refined grid solutions that we have presented is the appearance in the refined mesh 
solution of a recirculating boundary layer all across the bottom of the cavity. This suggests the possibility that a second grid dependent steady solution could appear before the critical point for the Hopf bifurcation. The grid dependent appearance of the recirculating boundary layer as a partially formed tertiary vortex in the bottom of the cavity does not seem to have a significant effect upon the essential periodic asymptotic dynamics in this Reynolds number range.

\section{3: Parameters}

Flows like the driven cavity can be thought of as three parameter bifurcation problems with a principal flow parameter such as Reynolds number, a principal geometry parameter such as aspect ratio, and a principal resolution or discretization parameter such as mesh size. Even though there are at least these three parameters that affect the cavity flow, we have concentrated here on the question of Hopf bifurcation with respect to the Reynolds number parameter with aspect ratio fixed at two. We conjecture that a Hopf bifurcation with respect to Reynolds number occurs in all lid driven cavities at all aspect ratios. If there is not a Hopf bifurcation for all aspect ratios, then it would be interesting to know the limits on aspect ratio for which there are Hopf bifurcations. If there are such limits, then we expect that they occur for small aspect ratios and not for large. A more general parametric investigation is underway. 


\section{7: Conclusions}

To summarize our numerical results for the aspect ratio two driven cavity:

1) On a coarse $48 \times 96$ grid at $R e=10000$ all measures indicate that the solution attains a periodic asymptotic state for $t \approx 1500$, with period $3.48 \leq T \leq 3.50$.

2] On a coarse $48 \times 96$ grid at $R e=5000$ all measures indicate that the solution attains a periodic asymptotic state for $t \approx 1100$, with period $2.469 \leq T \leq 2.484$. The asymptotic state for this flow is characterised by primary and secondary vortices in the upper and lower cavity, by periodic shedding of small tertiary counterrotating vortices off the downstream wall, by a wavy disturbance across the mid cavity shear layer, and by tertiary vortices in the bottom corners.

3) On a refined $96 \times 192$ grid at $R e=5000$ all measures indicate that the solution attains a periodic asymptotic state for $t \approx 3700$, with period $2.305 \leq T \leq 2.313$. The asymptotic state for this flow is characterised by primary and secondary vortices in the upper and lower cavity, by periodic shedding of small tertiary counterrotating vortices off the downstream wall, by a wavy disturbance across the mid cavity shear layer, by a partially formed third principle vortex at the bottom of the cavity, and by quartiary vortices in the bottom corners.

Based upon these computations we conjecture that the Navier-Stokes equations for the aspect ratio two driven cavity possess a Hopf bifurcation in the interval $2000 \leq R e \leq 5000$, since the transition has been computationally demonstrated.

Many interesting flow dynamics are shown by simulating the full time dependent flow history from the initial no flow state to the final periodic state shows. Particular examples are the development from early time of the periodic shedding of counterrotating vortex couples from the downstream wall, and the two unexpected transient oscillatory regimes before converging to a permanent periodic solution in the $R e=5000$ calculation with a $96 \times 192$ grid. Following the dynamical history of the flow helps in the interpretation of the final asymptotic state.

The investigation of the qualitative properties of unsteady flows treated as infinite dimensional dissipative dynamical systems is ushering in a new chapter of numerical anal- 
ysis. We have presented a number of qualitative measures and indicators that we have found to be useful in this type of study. In particular, we stress the dangers of relying totally upon any one of these measures. 


\section{Bibliography}

[1] P. Constantin and C. Foias, Navier-Stokes Equations (The University of Chicago Press, Chicago, 1988).

[2] P. Constantin, C. Foias and R. Temam, SLAM J. Numer. Anal. 21 , 615 (1984).

[3] A. Fortin, M. Fortin and J. Gervais, J. Comput. Phys. 70,295 (1987).

[4] U. Ghia, K. N. Ghia and C. T. Shin, J. Comput. Phys. 48,387 (1982).

[5] R. Glowinski, H. Keller and L. Rinehart, SIAM J. Sci. Stat. Computing 6 , 793 (1985).

[6] J. W. Goodrich, in preparation.

[7] J. W. Goodrich and W. Y.Soh, J. Comput. Phys. , to appear (1989).

[8] K. Gustafson and K. Halasi, J. Comput. Phys. 64, 279 (1986).

[9] K. Gustafson and K. Halasi, J. Comput. Phys. 70 , 271 (1987).

[10] K. Gustafson and R. Leben, "Vortex Subdomains", in Proc. First Intern. Symp. on Domain Decomposition Methods for Partial Differential Equations, Ed: R. Glowinski, G. Golub, G. Meurant and J. Periaux, 370-380 (SIAM, Philadelphia, 1988).

[11] J. K. Hale, X.-B. Lin and G. Raugel, Math. Comput. 50 , 89 (1988).

[12] J. G. Heywood and R. Rannacher, SIAM J. Numer. Anal. 19,275 (1982).

[13] J. G. Heywood and R. Rannacher, SIAM J. Numer. Anal. 23,750 (1986).

[14] J. G. Heywood and R. Rannacher, SIAM J. Numer. Anal. 25,489 (1988).

[15] J. Linden, "A Multigrid Method for Solving the Biharmonic Equation on Rectangular Domains," Arbeitspapiere der GMD No. 143, Gesellschaft für Mathematik und Datenverarbeitung, St. Augustin, 1985.

[16] S. Paolucci and D. R. Chenoweth, J. Fluid Mech. 201,379 (1989).

[17] R. Schreiber and H. Keller, J. Comput. Phys. 49,310 (1983).

[18] R. Schreiber and H. Keller, J. Comput. Phys. 49,165 (1983).

[19] R. Temam, Infinite-Dimensional Dynamical Systems in Mechanics and Physics, (Springer-Verlag, New York, 1988). 
LID VELOCITY

$$
\Psi(x, 2, t)=0 \text { AND } \frac{\partial \psi}{\partial y}(x, 2,1)=1 \text { FOR } 0<t
$$

$\psi(x, y, 0)=0$ FOR $0=t$

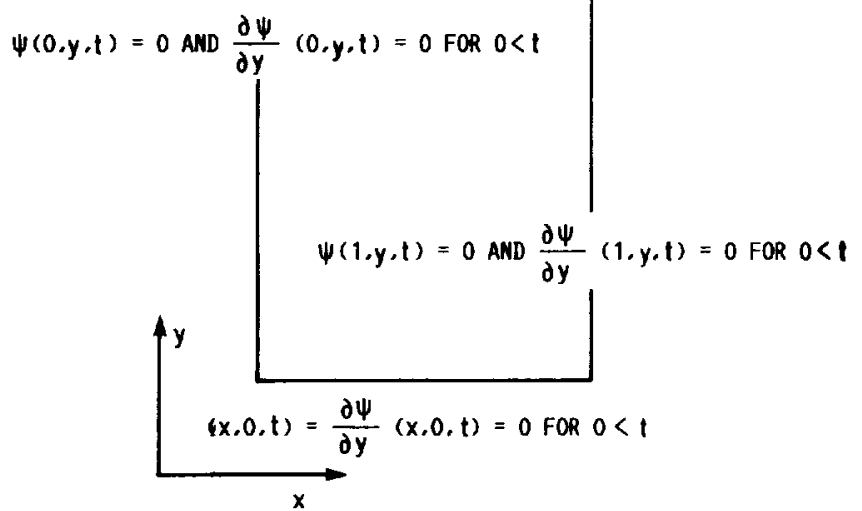

FIGURE 1. - TWO DIMEMSIOMAL COMPUTATIONAL DOMAIN, $0 \leq x \leq 1$ AMD 0 $\leq y \leq 2$.

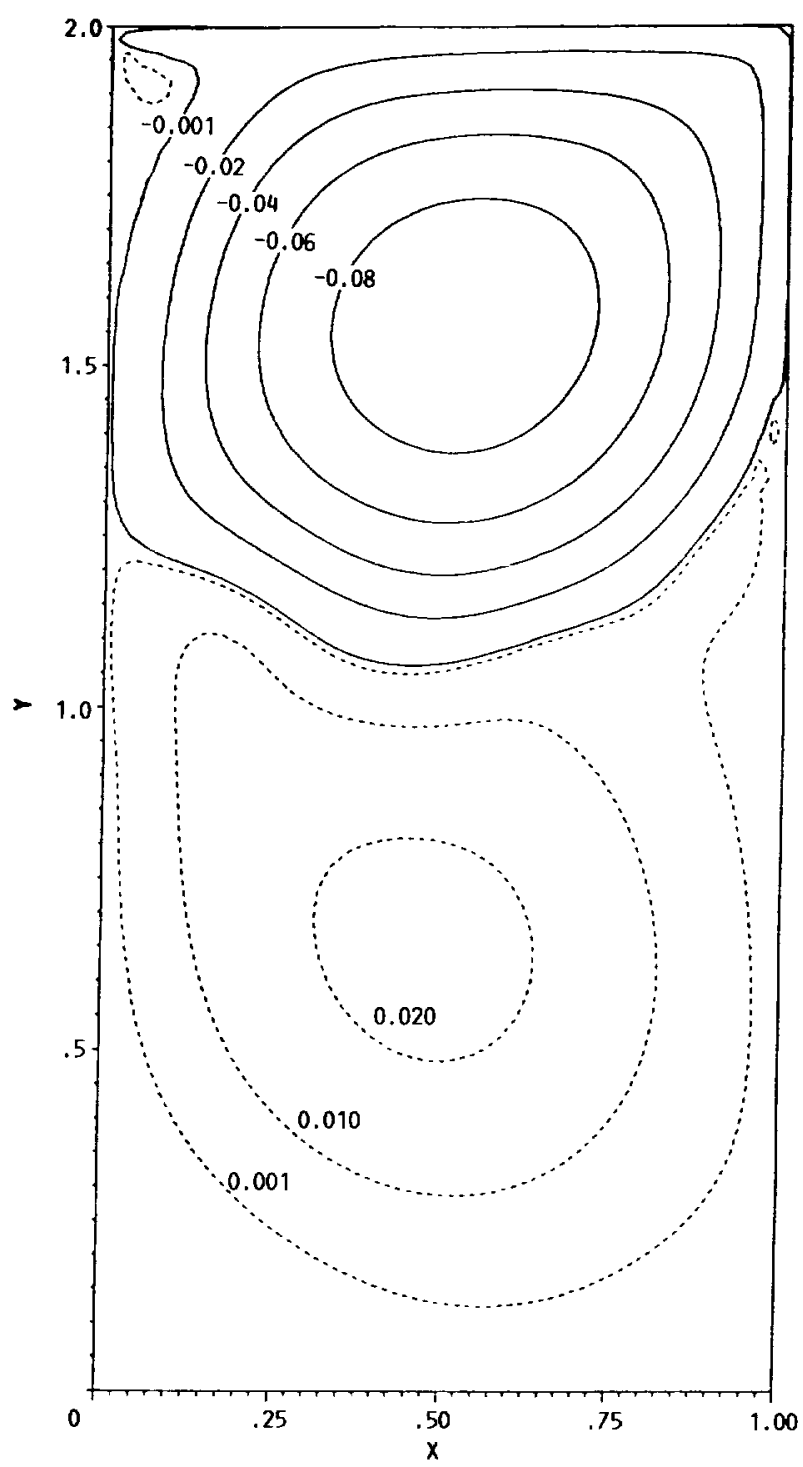

FIGURE 2. - STREAMFUNCTION CONTOUR PLOT FOR $t=4000$ WITH Re $=5000$ ON A $48 \times 96$ GRID. 


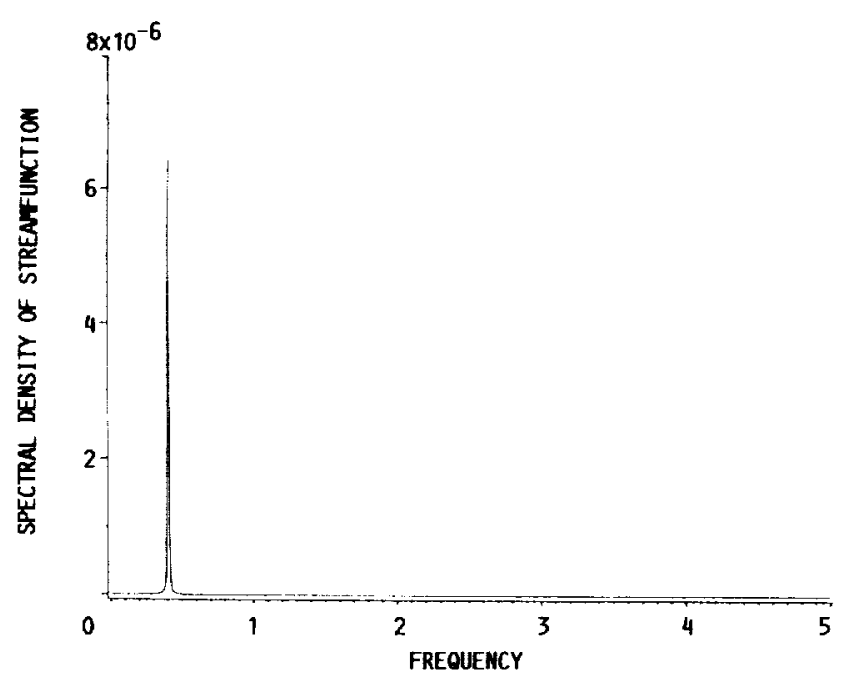

(A) SPECTRAL DENSITY FOR STREAHFUNCTION.

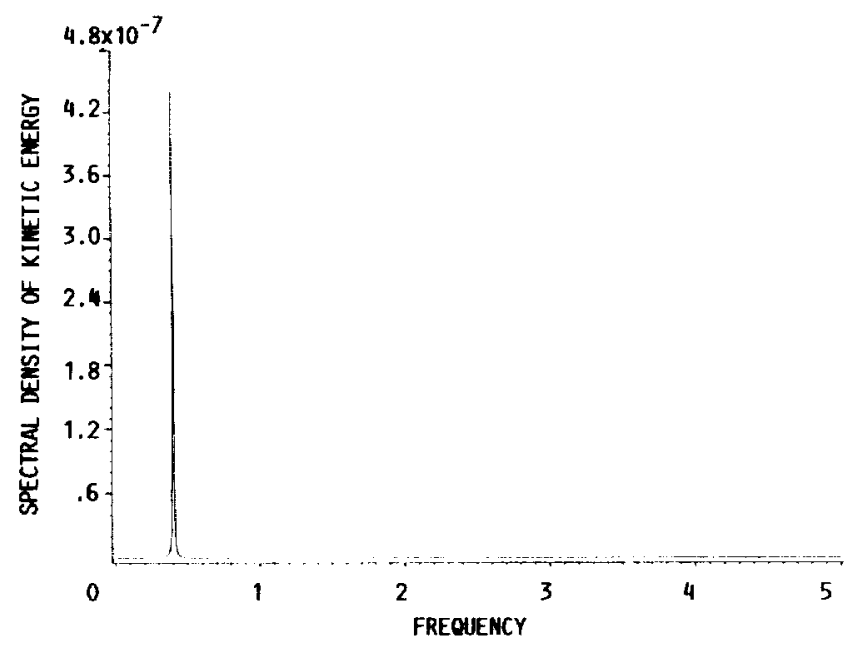

(C) SPECTRAL DENSITY FOR KIMETIC EMERGY.

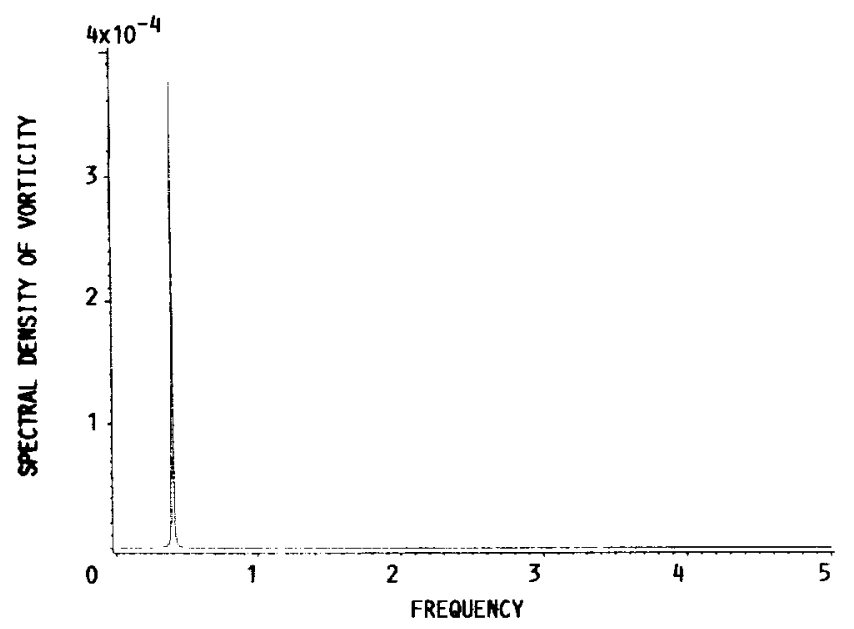

(E) SPECTRAL DENSITY FOR VORTICITY.

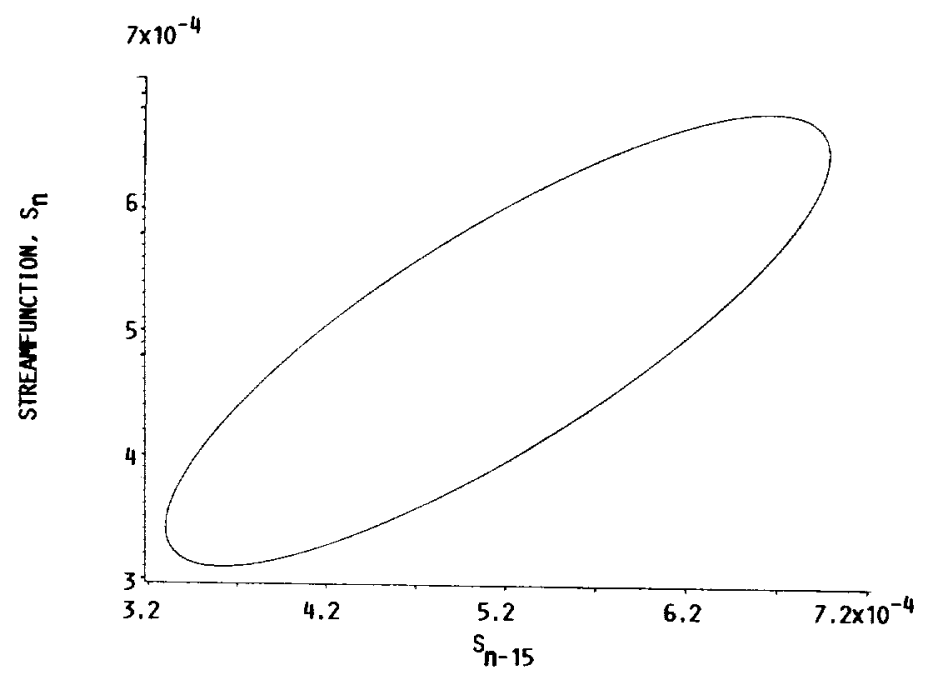

(B) STREAMFUNCTION PHASE PORTRAIT, 15 IIME STEP LAG.

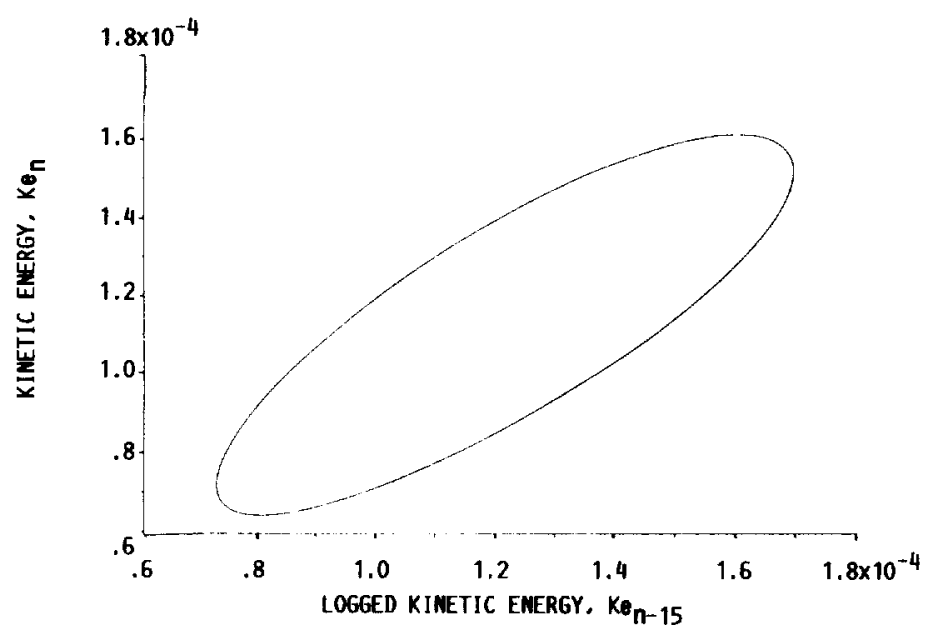

(D) KINETIC ENERGY PHASE PORTRAIT, 15 TIME STEP LAG.

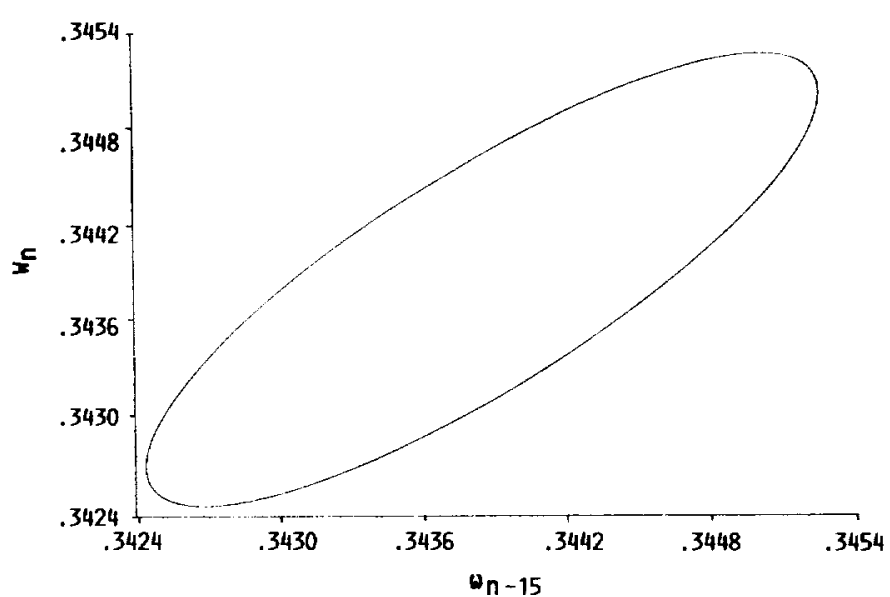

(F) VORTICITY PHASE PORTRAIT, 15 TIME STEP LAG.

FIGURE 3. - DATA AT $(1, j)=(44.50)$ FOR $4000 \leq t \leq 4100$, WITH Re $=5000$ ON A $48 \times 96$ GRID. 


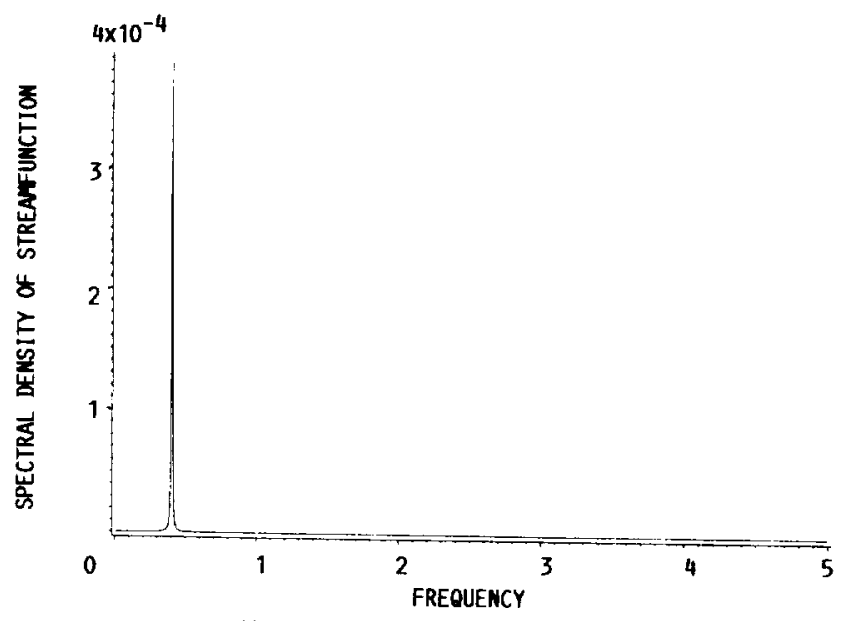

(A) SPECTRAL DENSITY OF STREAWFUNCIION.

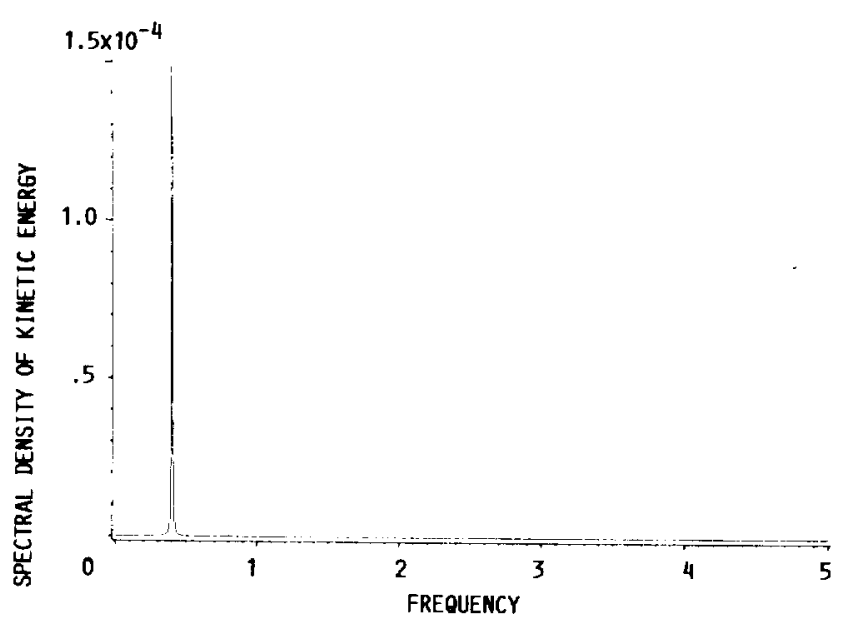

(C) SPECTRAL DENSITY OF KINETIC ENERGY.

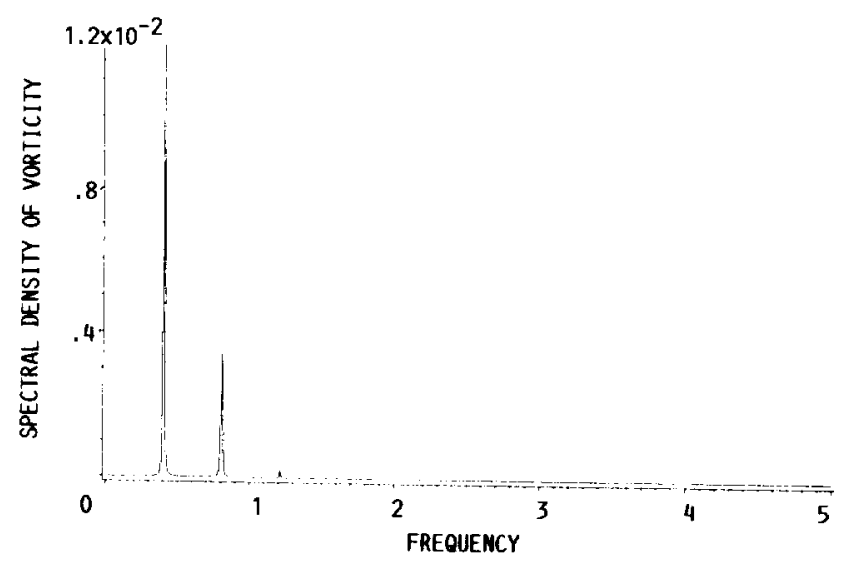

(E) SPECTRAL DENSITY OF VORTICITY.

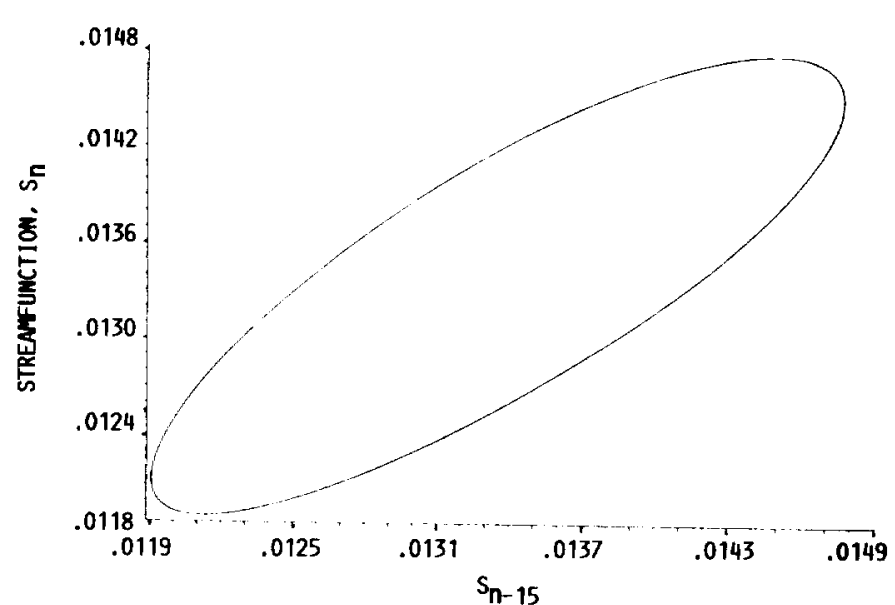

(B) STREAFFUNCTION PHASE PORTRAIT, 15 TIME STEP LAG.

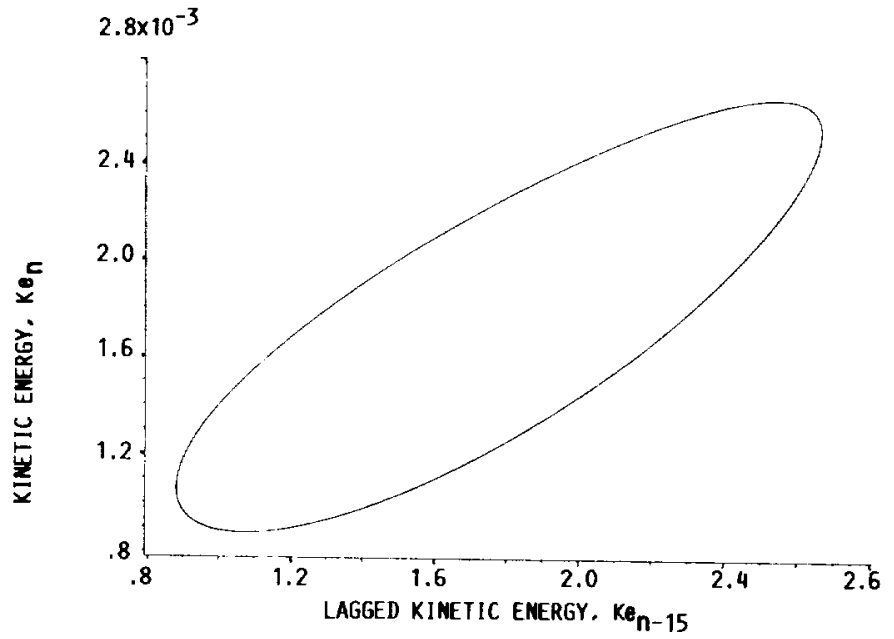

(D) KINETIC EMERGY PHASE PORTRAIT, 15 TINE STEP LAG.

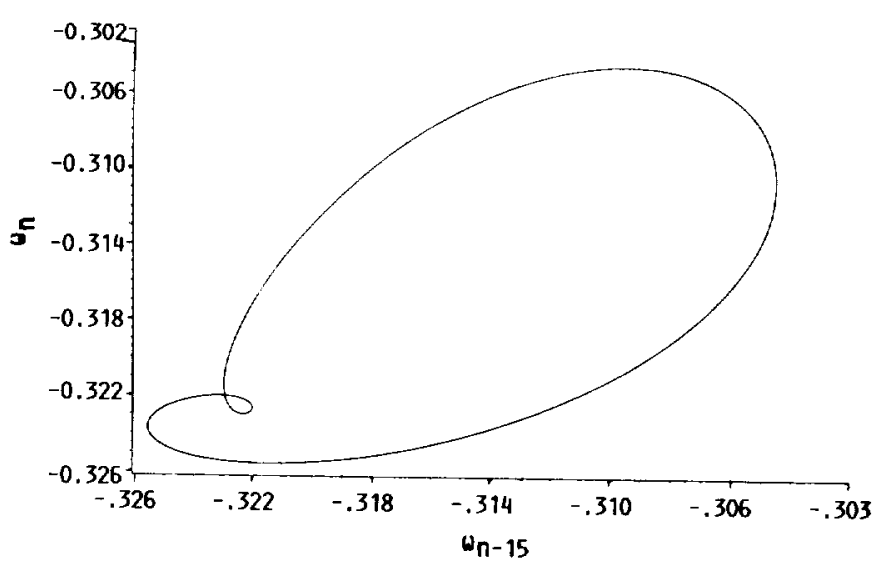

(F) VORTICITY PHASE PORTRAIT, 15 TIME STEP LAG.

FIGURE 4. - DATA AT $(1,1)=(16,46)$ FOR $4000<1 \leq 4100$, WITH Re $=5000$ ON A $48 \times 96$ GRID. 


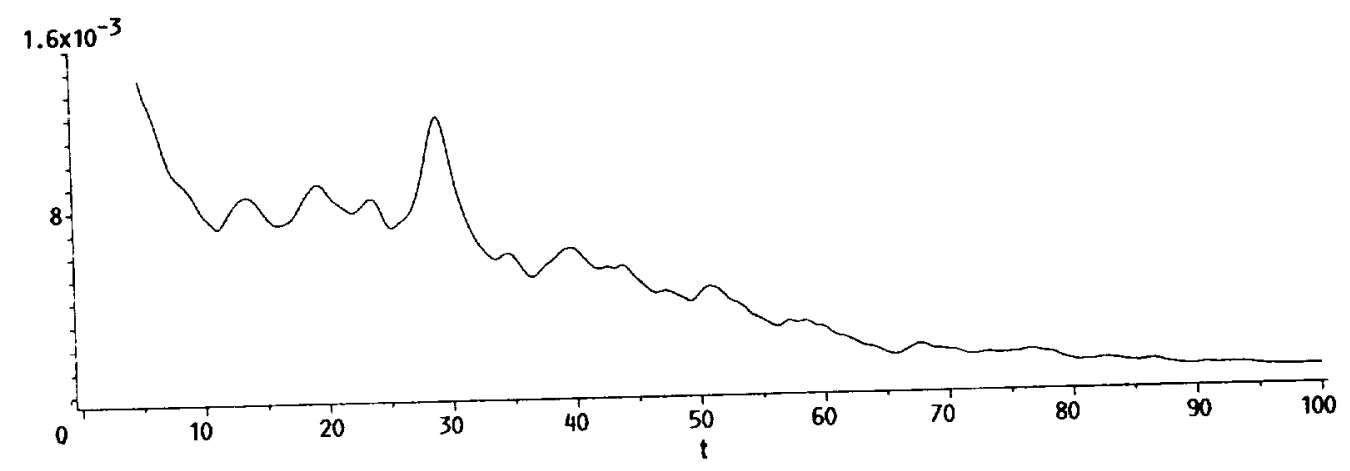

(A) $5 \leq t \leq 100$.

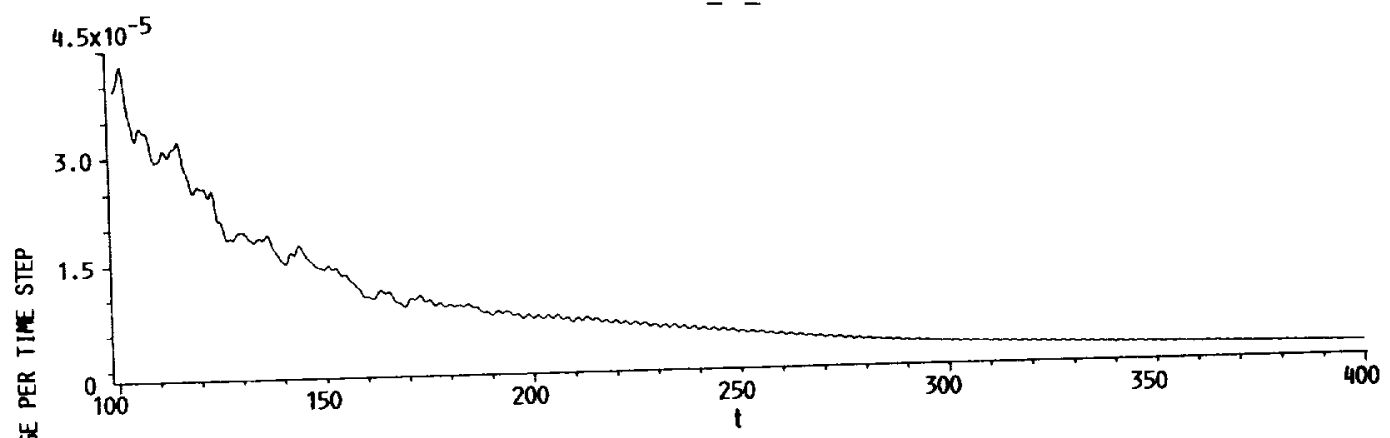

(B) $100<1 \leq 400$.

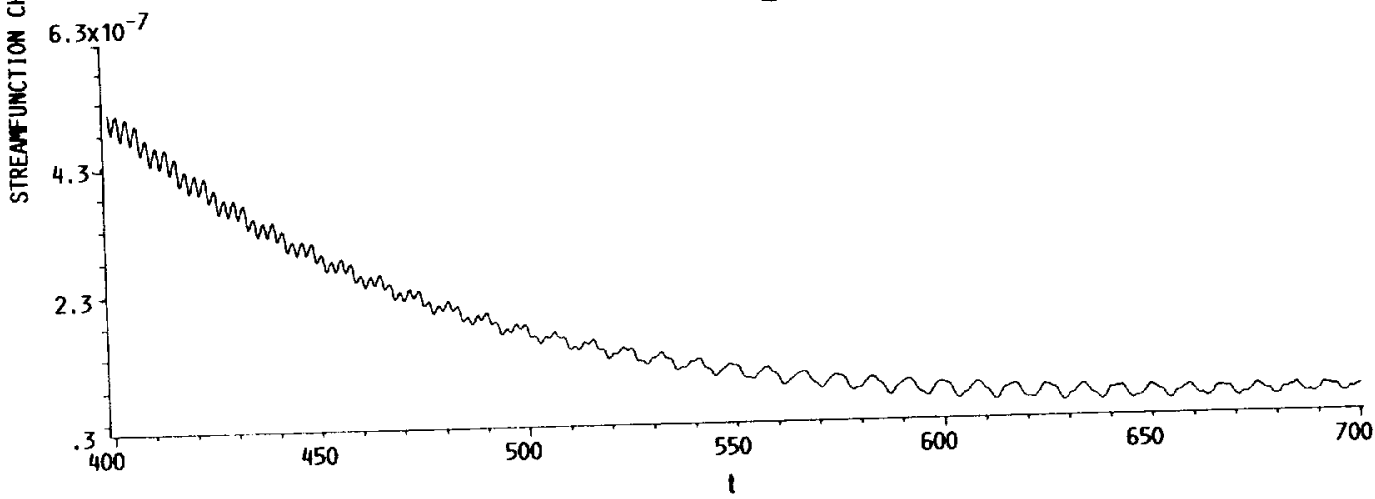

(C) $400<t \leq 700$.

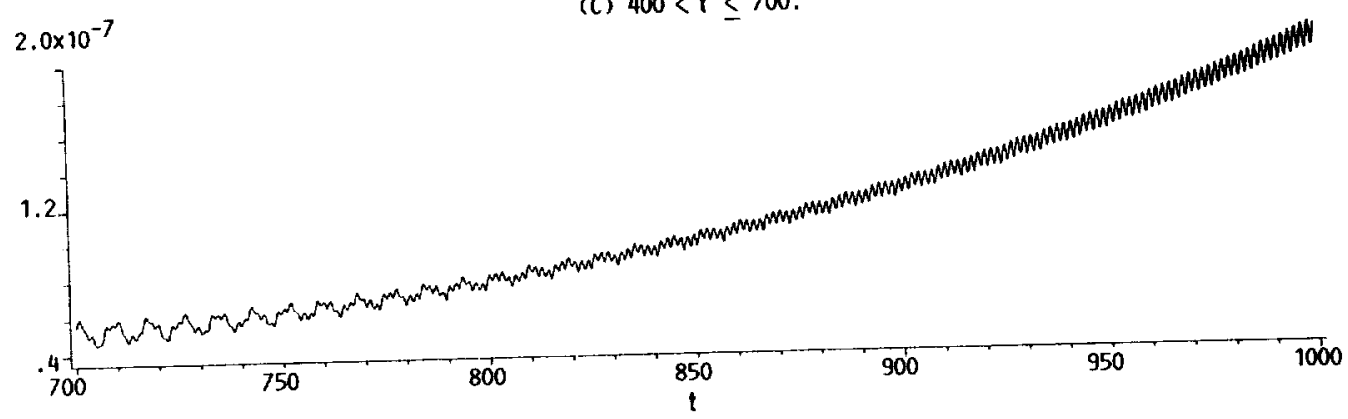

(D) $700<t \leq 1000$.

FIGURE 5. - RELATIVE $L_{1}$ MORM OF STREAMFUNCTION CHANGE Ro $=5000.96 \times 192$ GRID. 

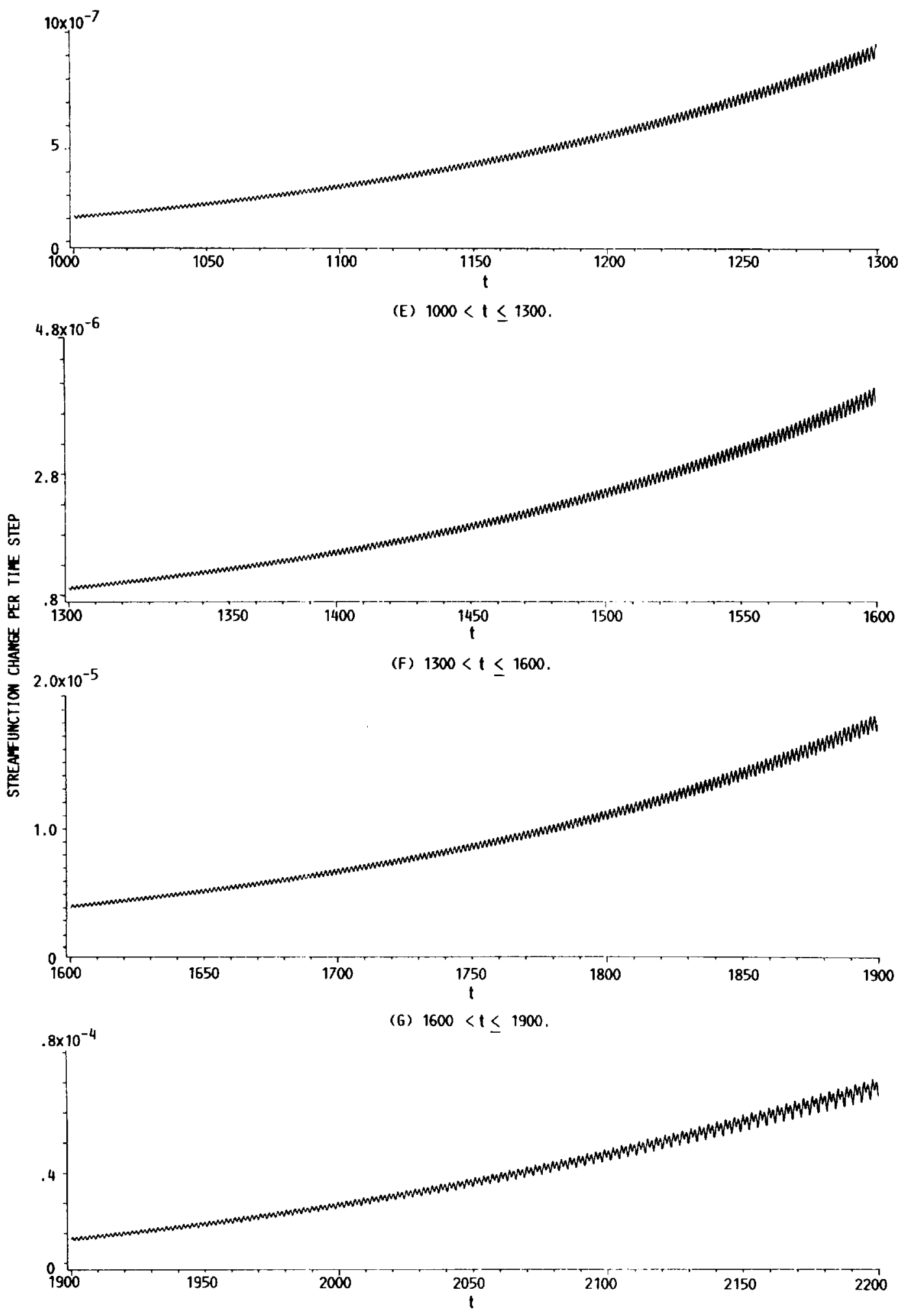

(H) $1900<t \leq 2200$.

FIGURE 5, - COMTIMUED. 


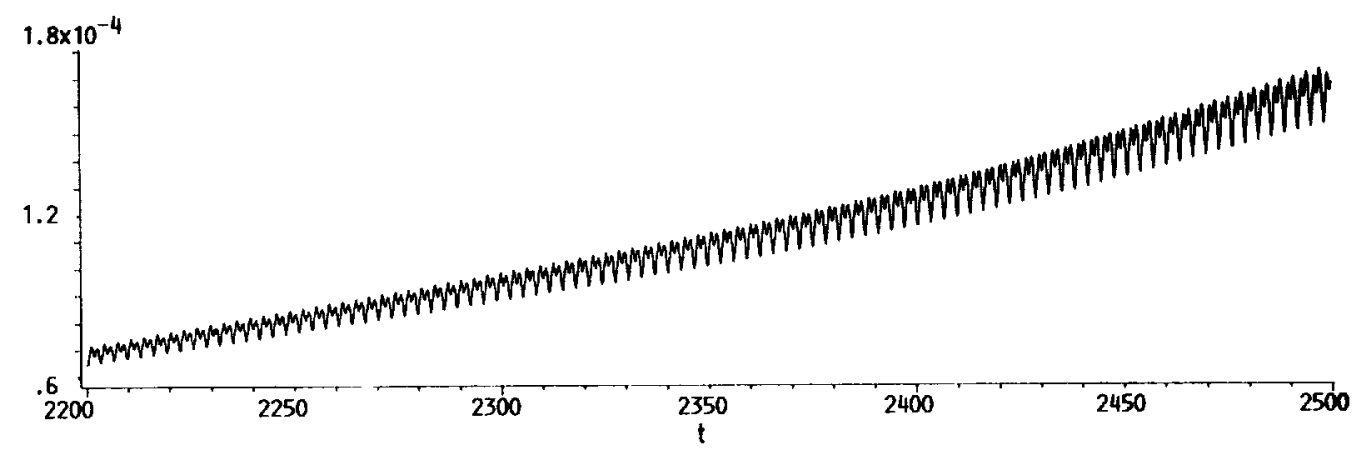

(I) $2200<t \leq 2500$.
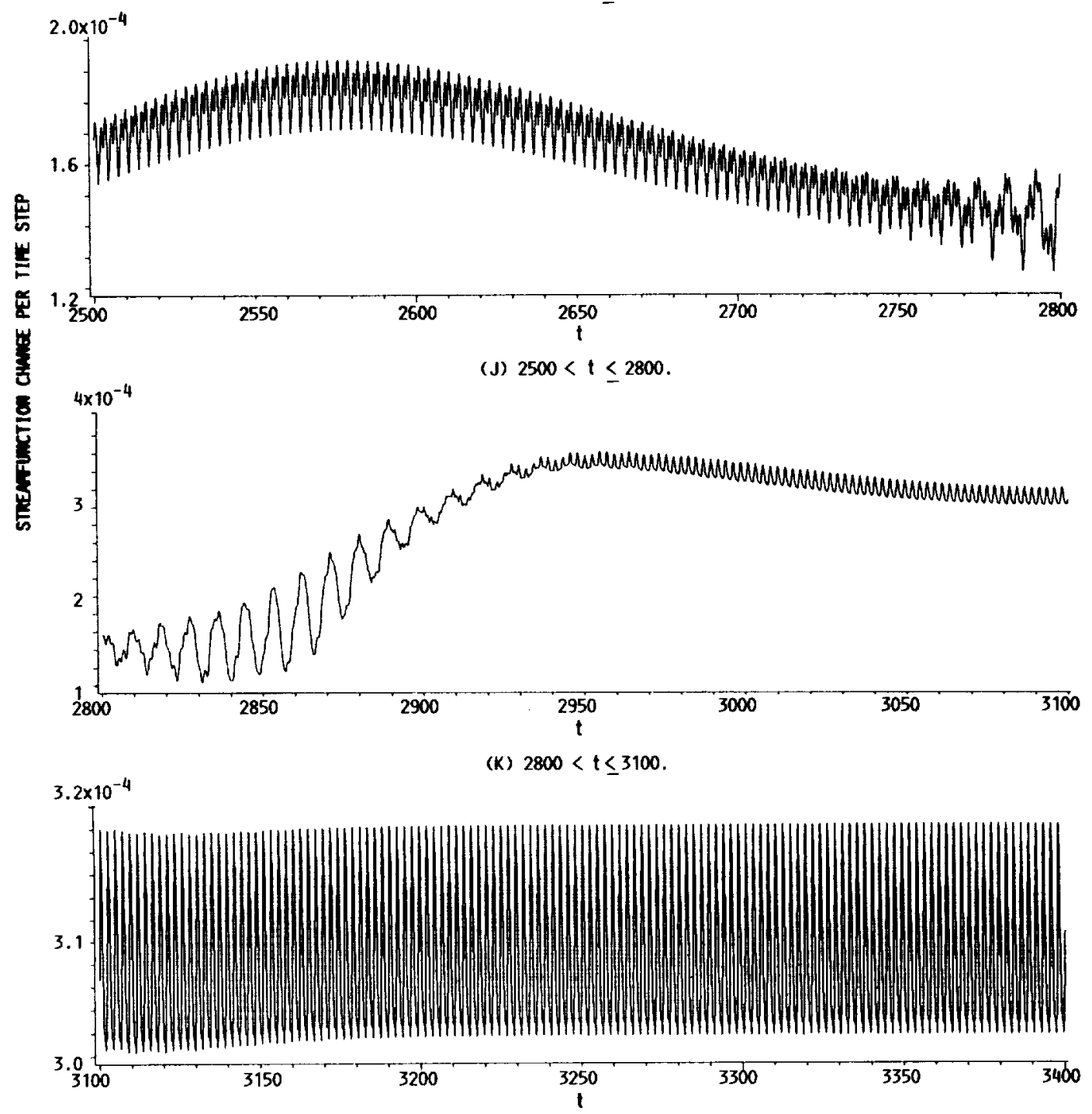

(L) $3100<t \leq 3400$.

FIGURE 5. - COMTIMUED. 

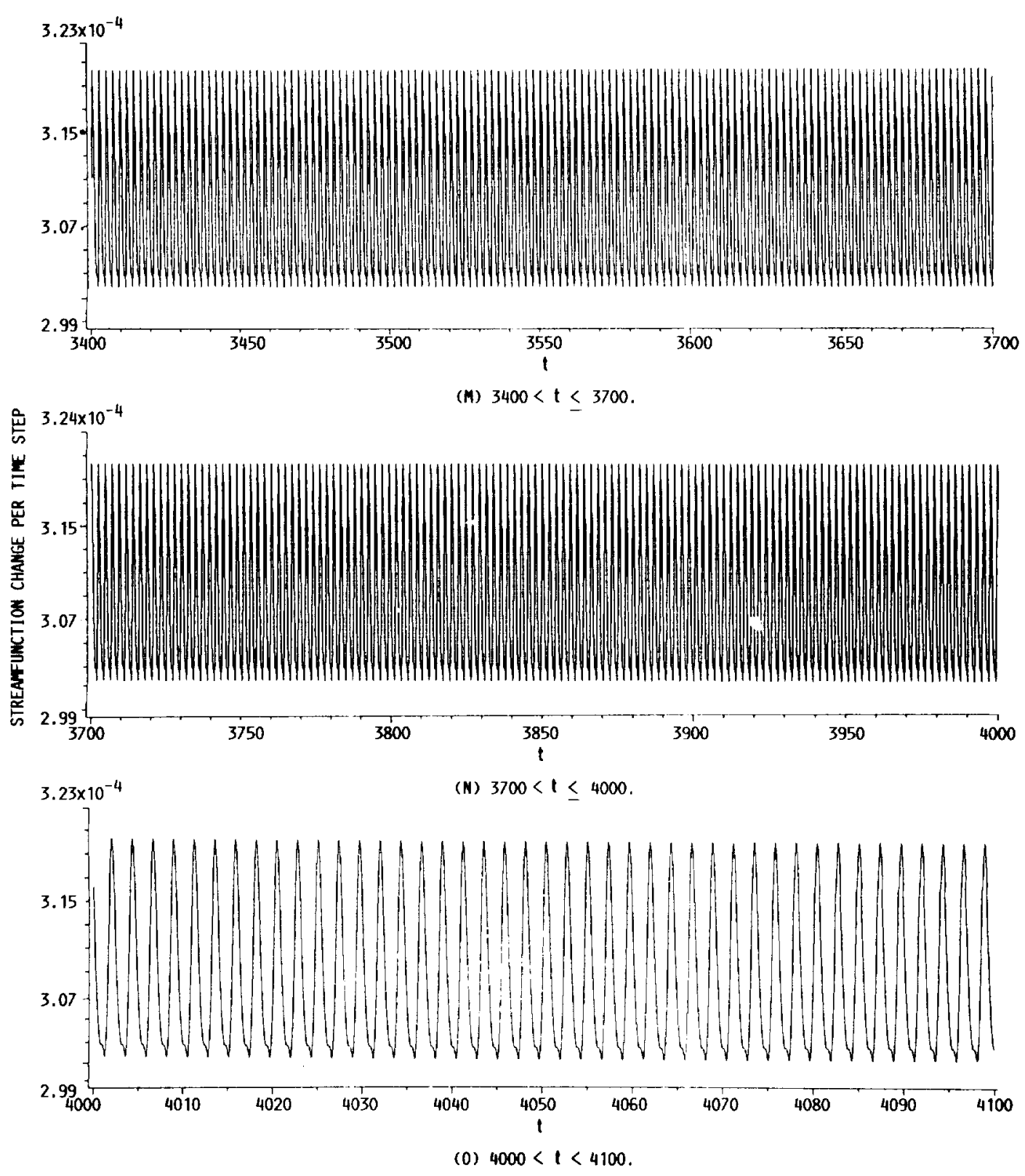

FIGURE 5. - CONCLUDED. 


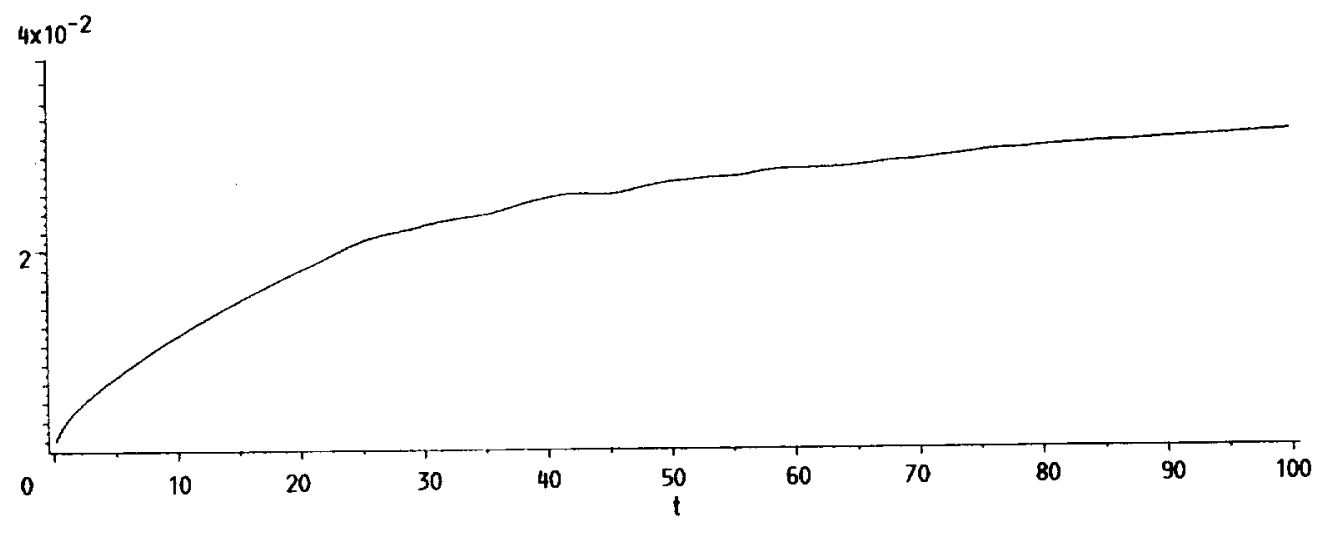

(A) $5<1 \leq 100$.

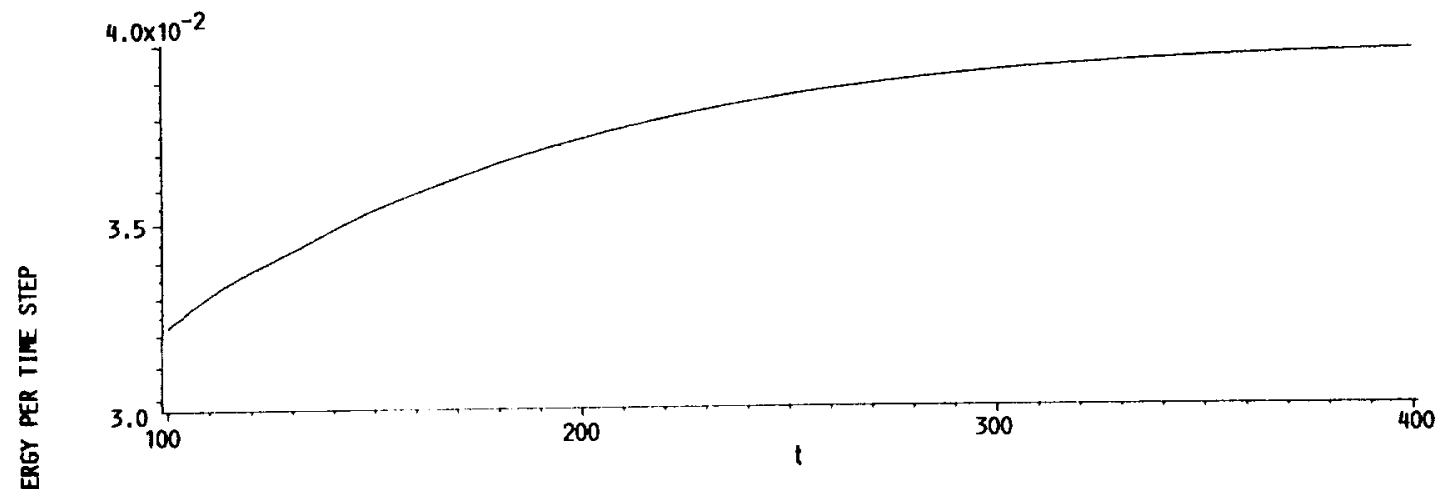

(B) $100<1 \leq 400$.

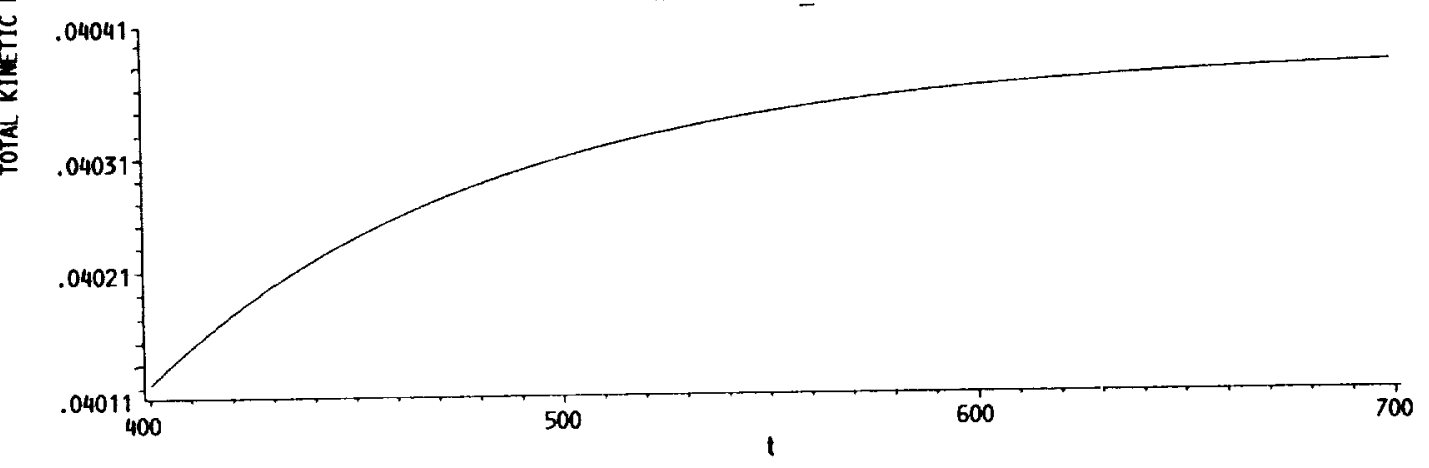

(C) $400<t \leq 700$.

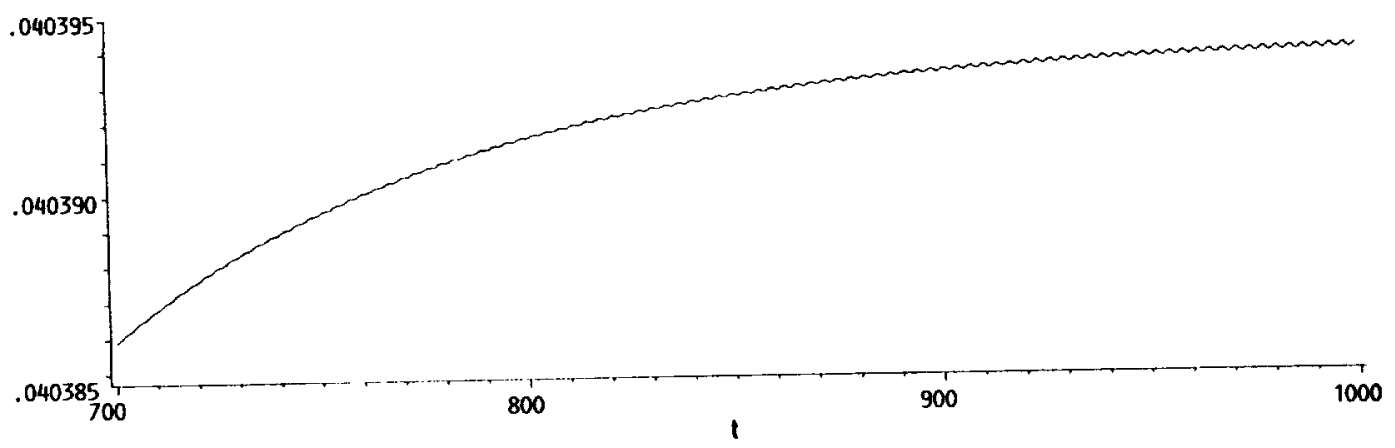

(D) $700<t<1000$.

FIGIRE 6. - TOTAL KIMETIC EMERGY, Re $=5000,96 \times 192$ GRID. 

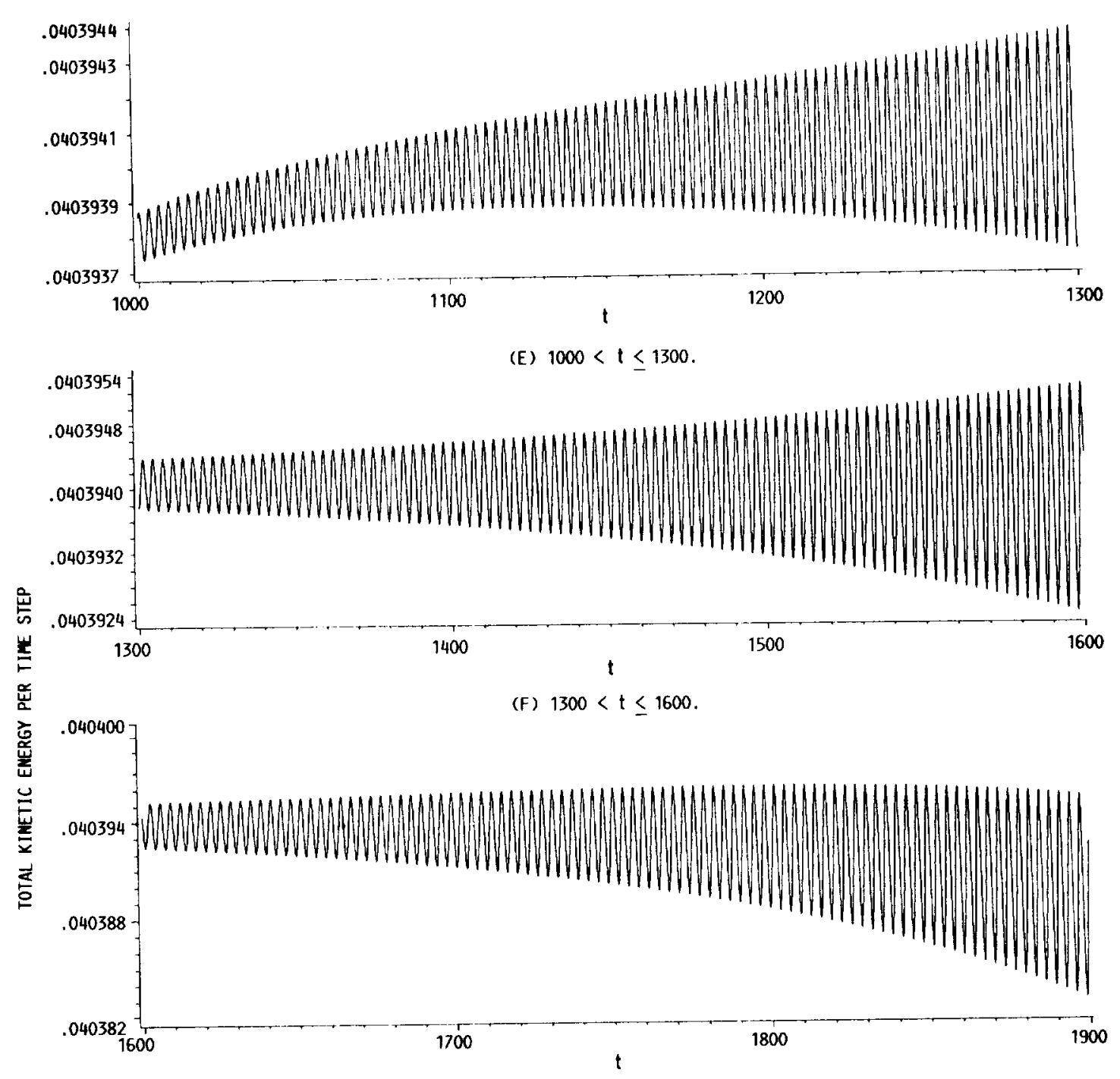

(G) $1600<t \leq 1900$.

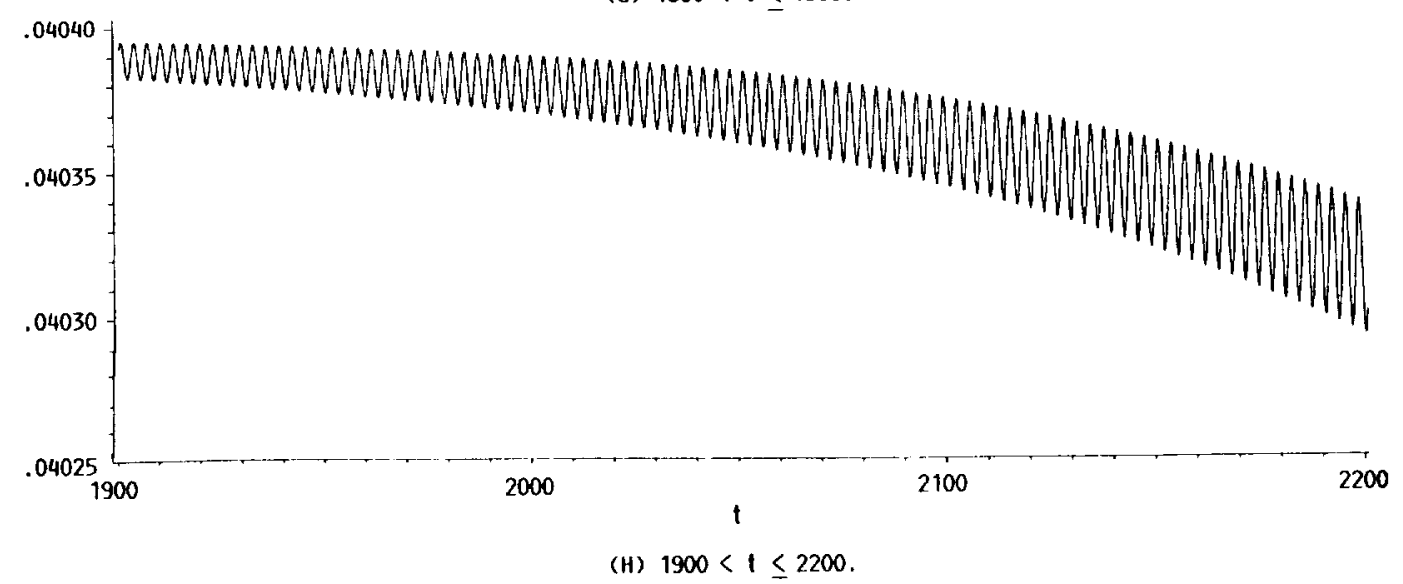

FIGURE 6. - CONTINUED. 

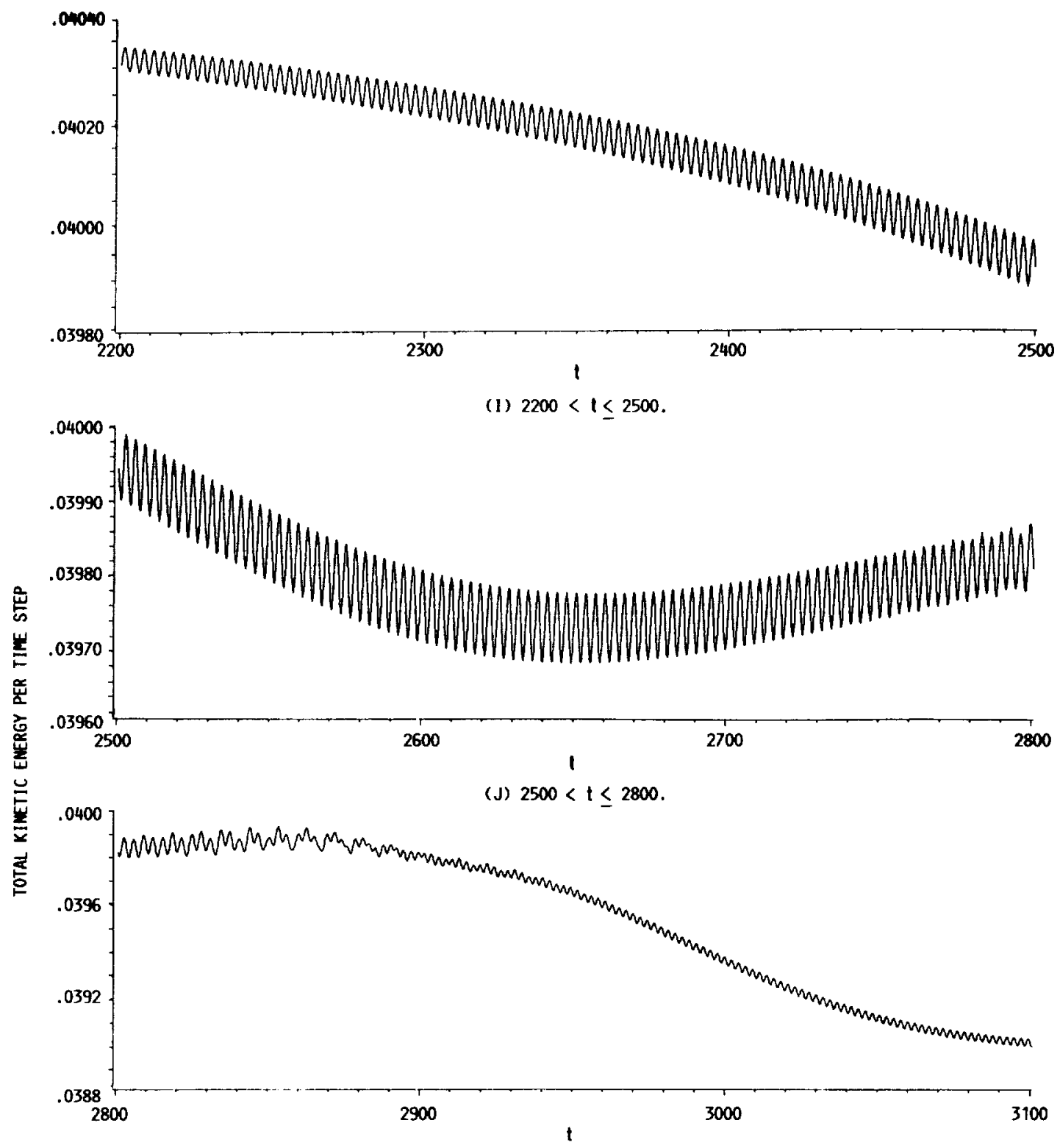

(K) $2800<t<3100$.

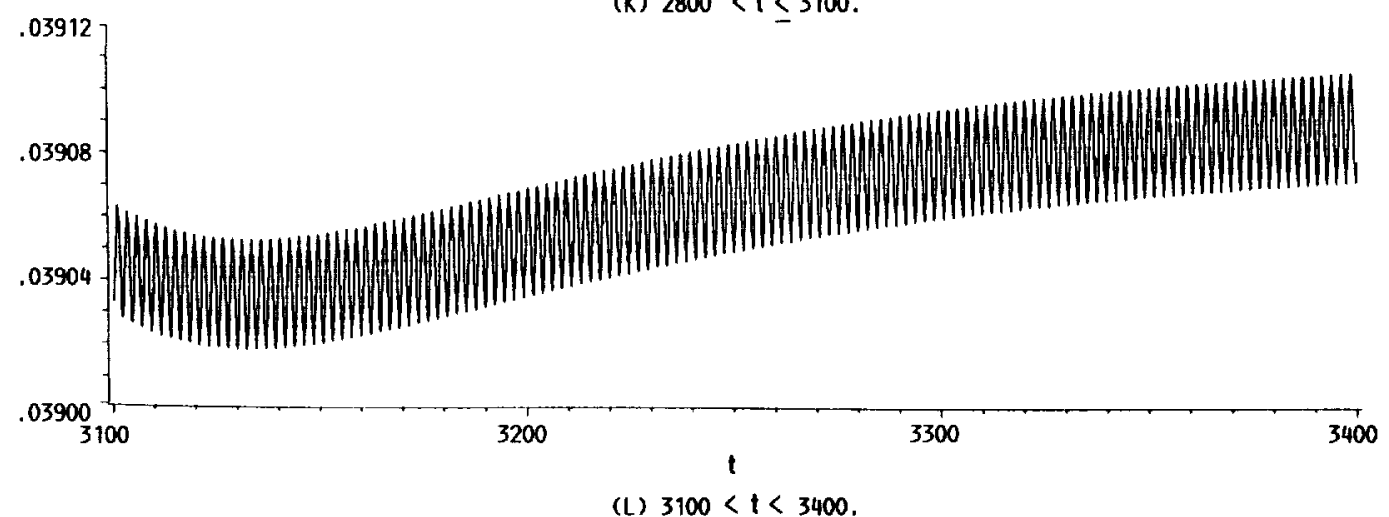

(L) $3100<t \leq 3400$. 

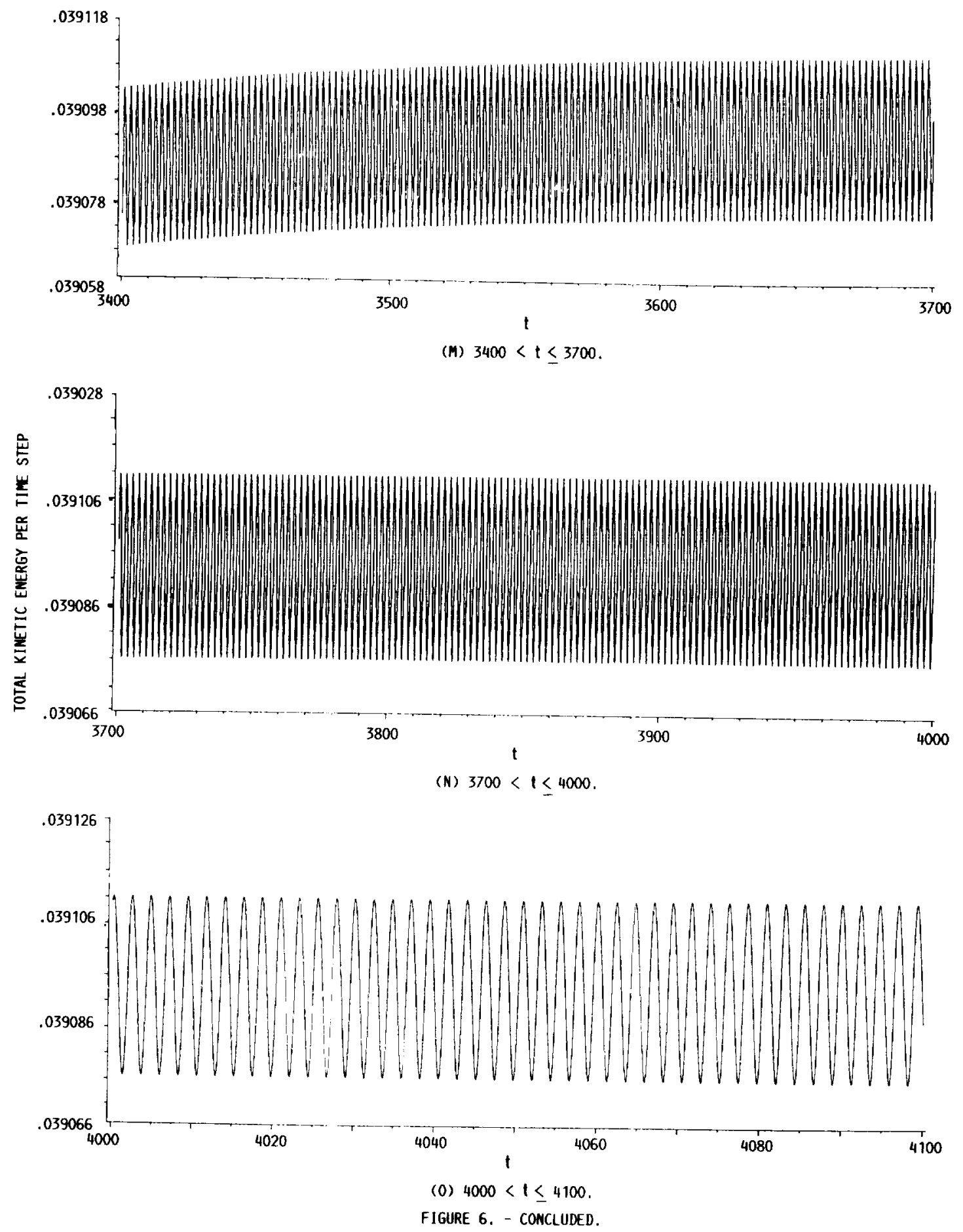


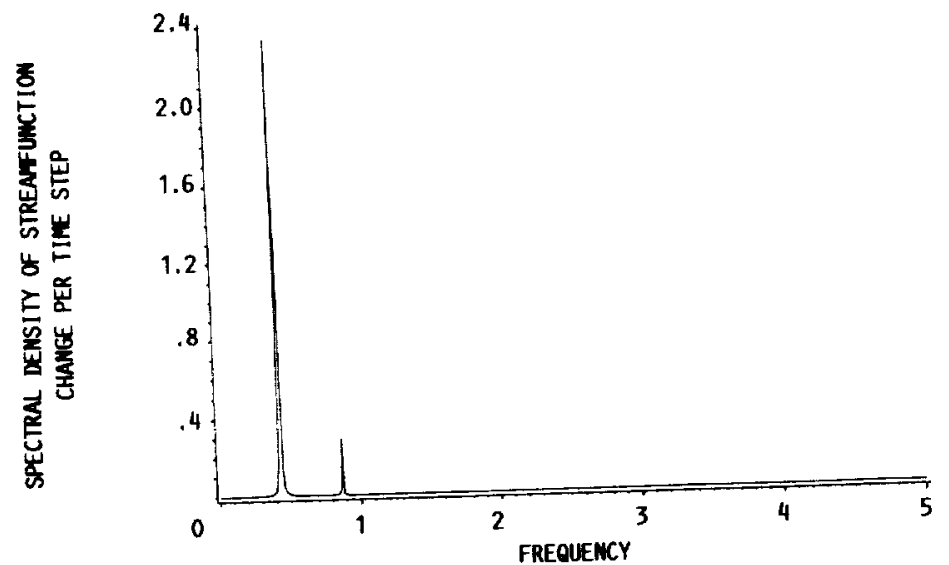

(A) SPECTRAL DENSITY FOR RELATIVE $L_{1}$ MORM OF STREAMFUMCTIOM CHANGE.

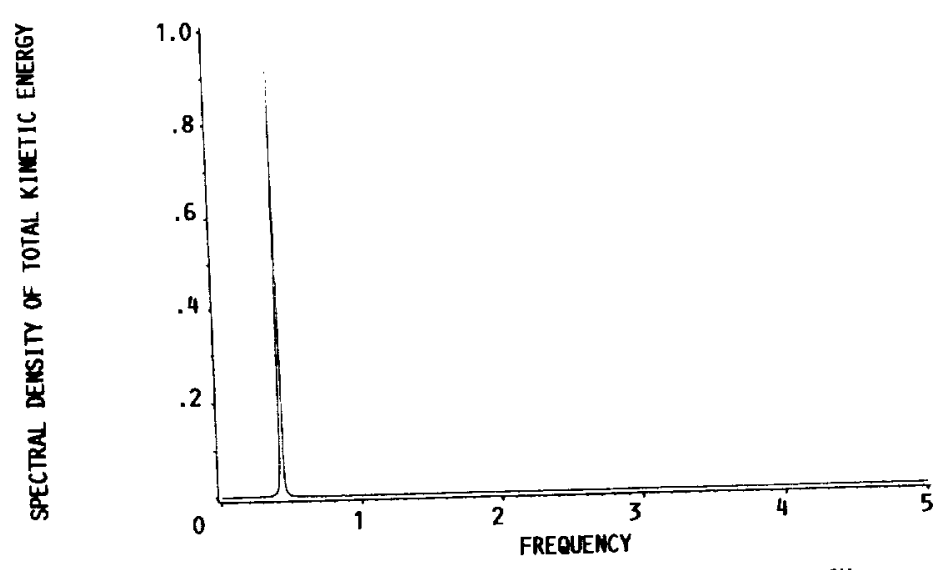

(B) SPECTRAL DENSITY FOR TOTAL KINETIC ENERGY.

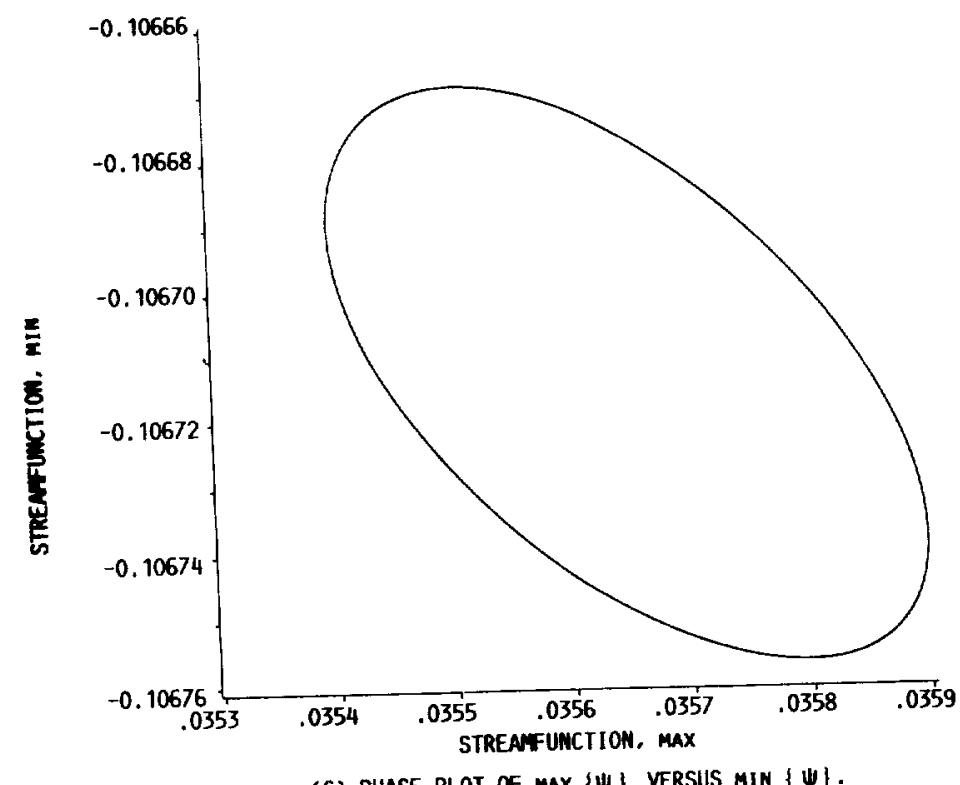

(C) PHASE PLOT OF max $\{\Psi\}$ VERSUS MIN $\{\Psi\}$.

FIGURE 7. - GLOBAL DATA FOR Re $=5000$ ON A $96 \times 192$ GRID WITH $4000<t \leq 4100$. 


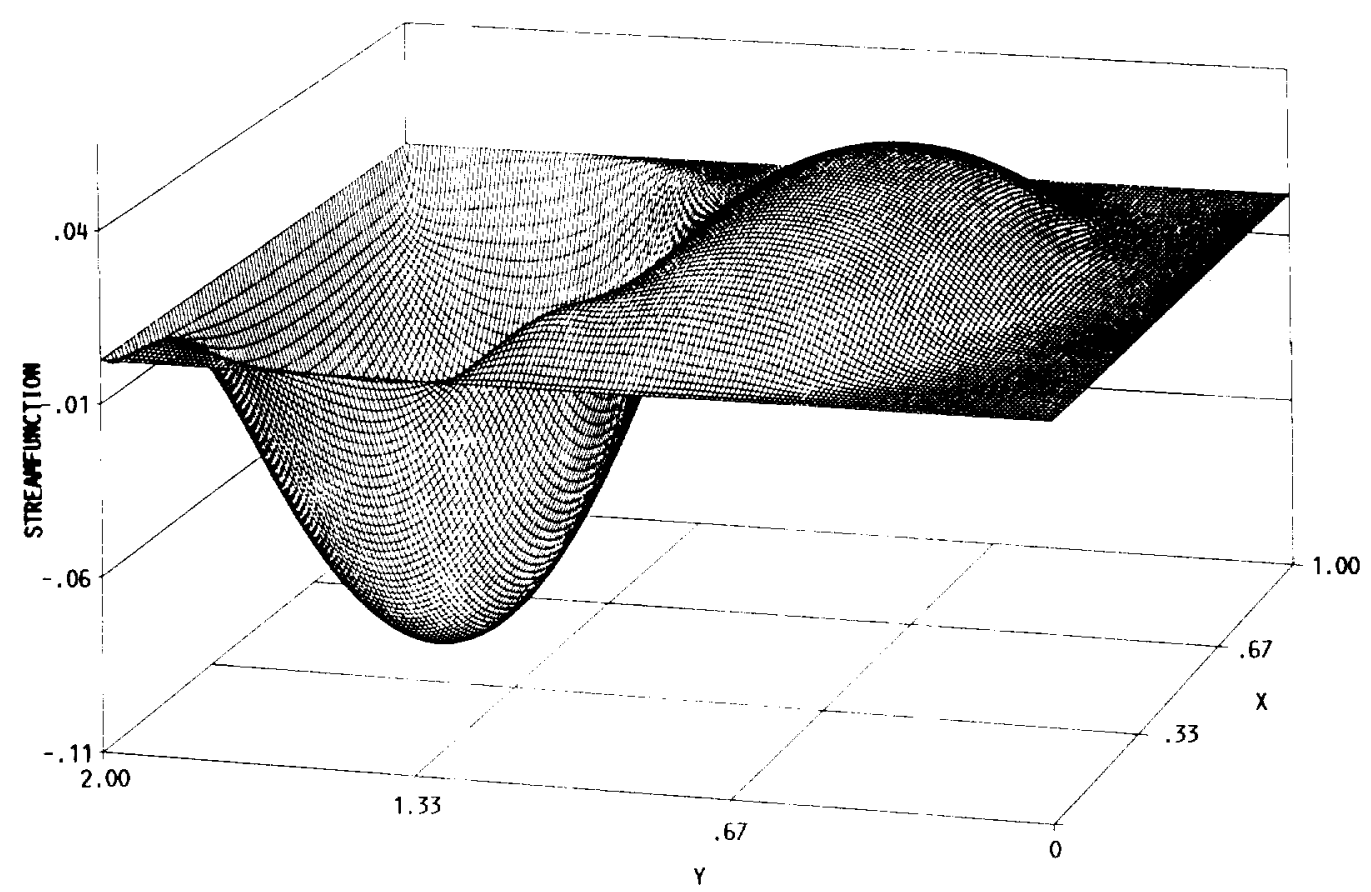

(A) SURFACE PLOT OF STREAMFUNCTION.

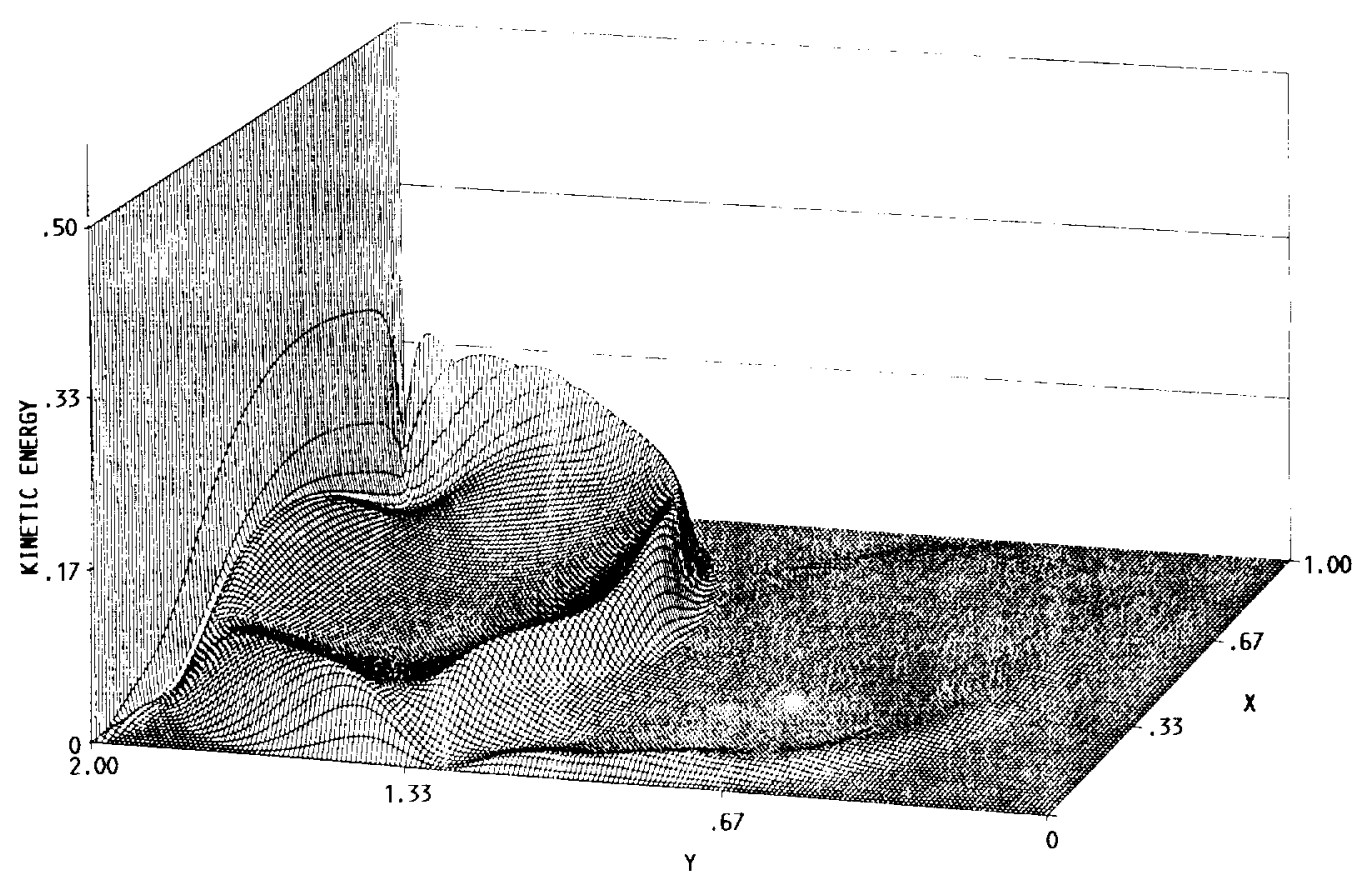

(B) SURFACE PLOT OF KINETIC ENERGY.

FIGURE 8. - FLOW FIELD OF Re $=5000$ ON A $96 \times 192$ GRID AT $t=4100$. 


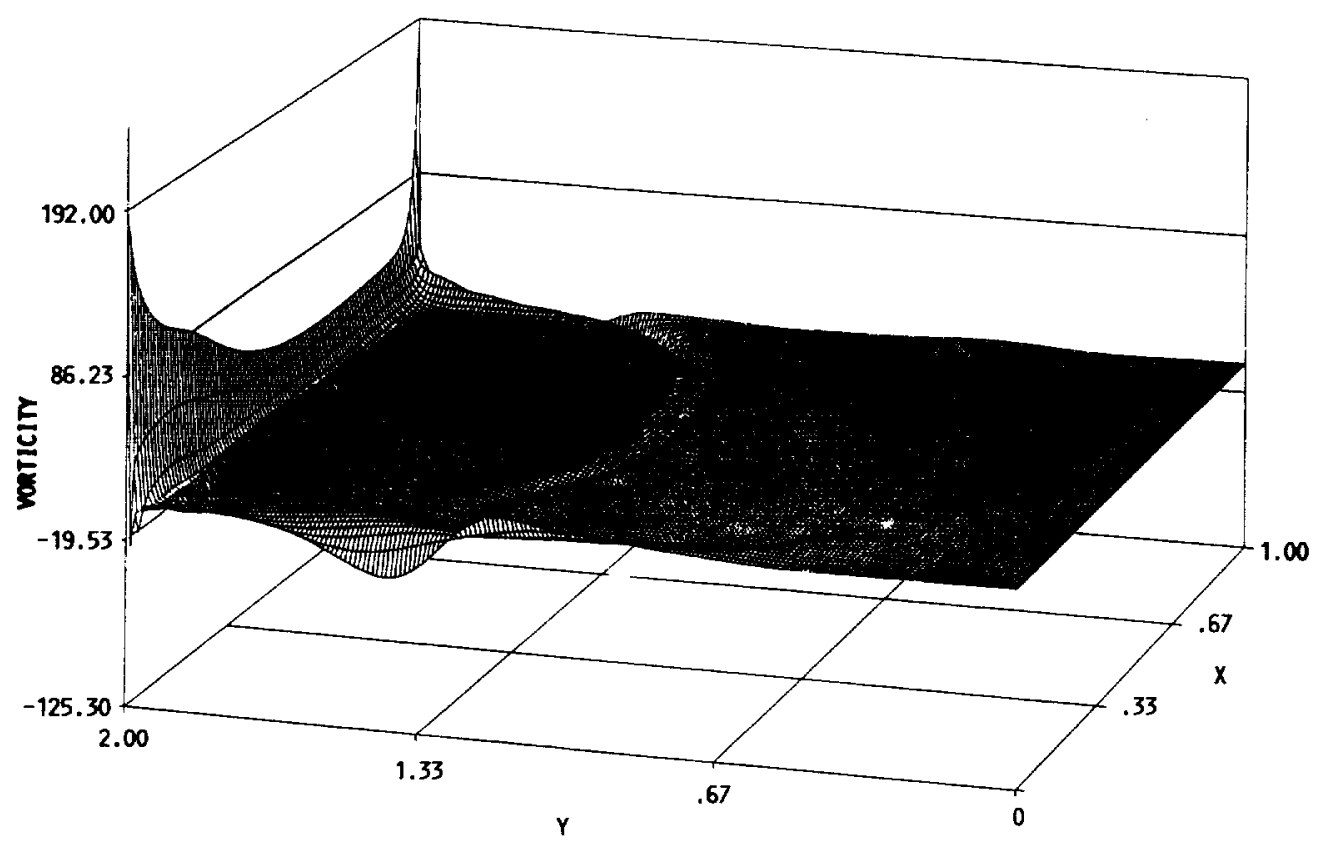

(C) SURFACE PLOT OF VORTICITY.

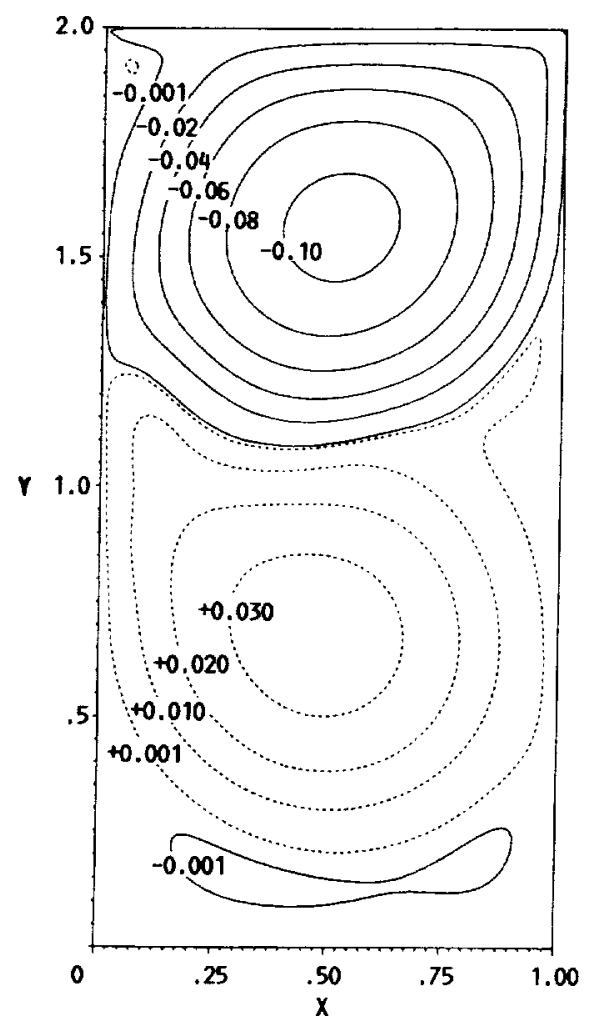

(D) STREAMFUMCTION CONTOUR PLOT.

FIGURE 8. - COMCLUDED. 


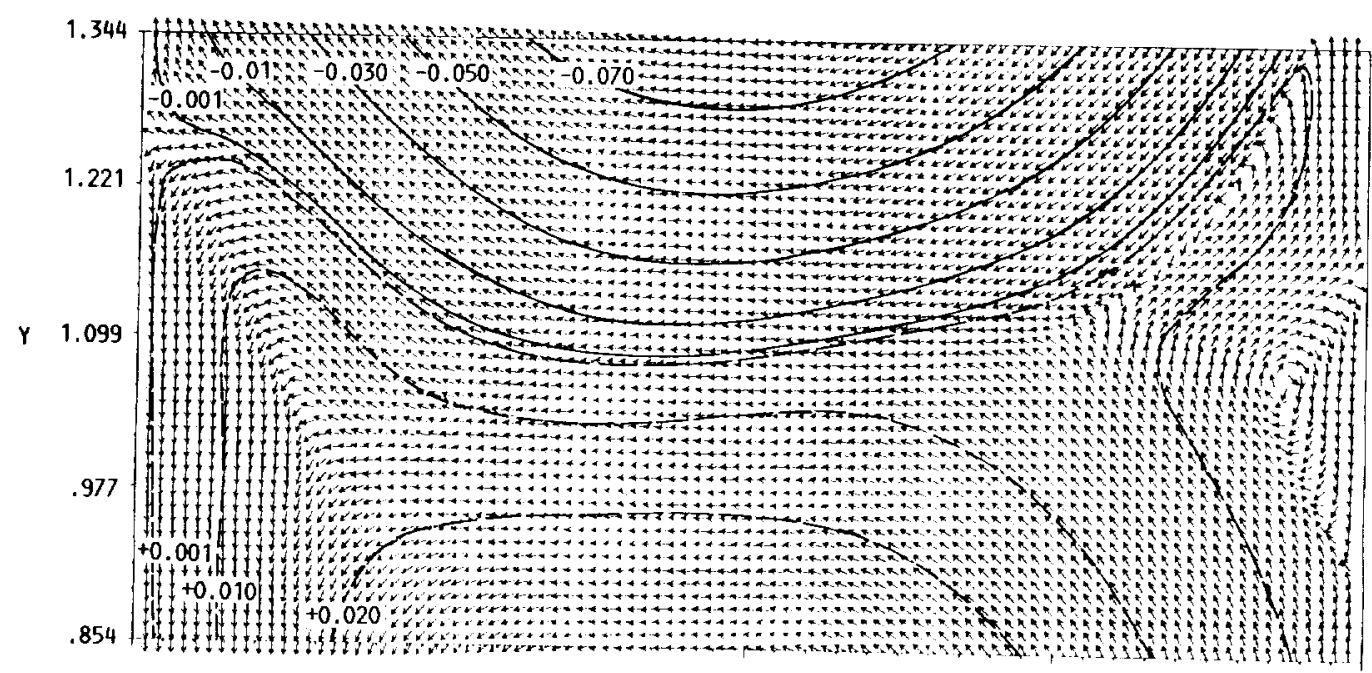

(A) $t=4100.00$

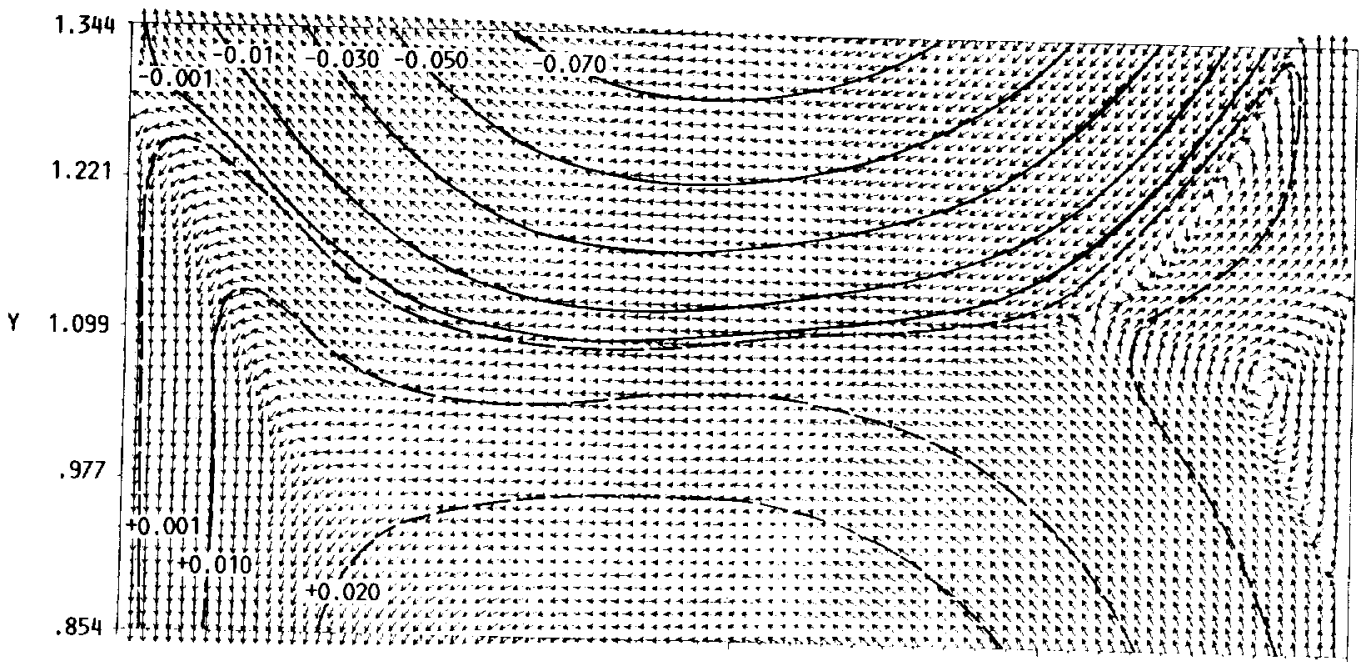

(B) $1=4100.25$

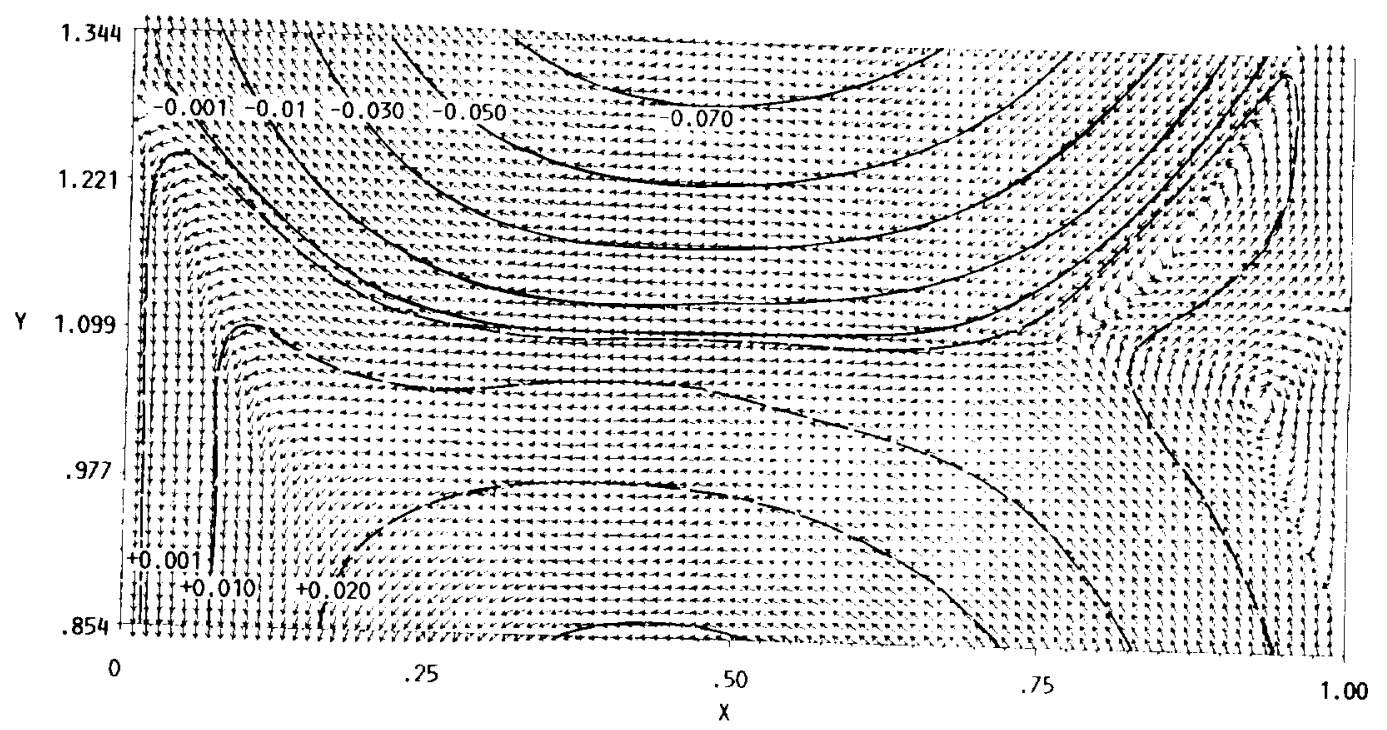

(C) $t=4100.50$.

FIGURE 9. - STREAMFUNCTION CONTOURS WITH DIRECTIONAL VECTOR PLOIS FOR ONF CYCLE. 


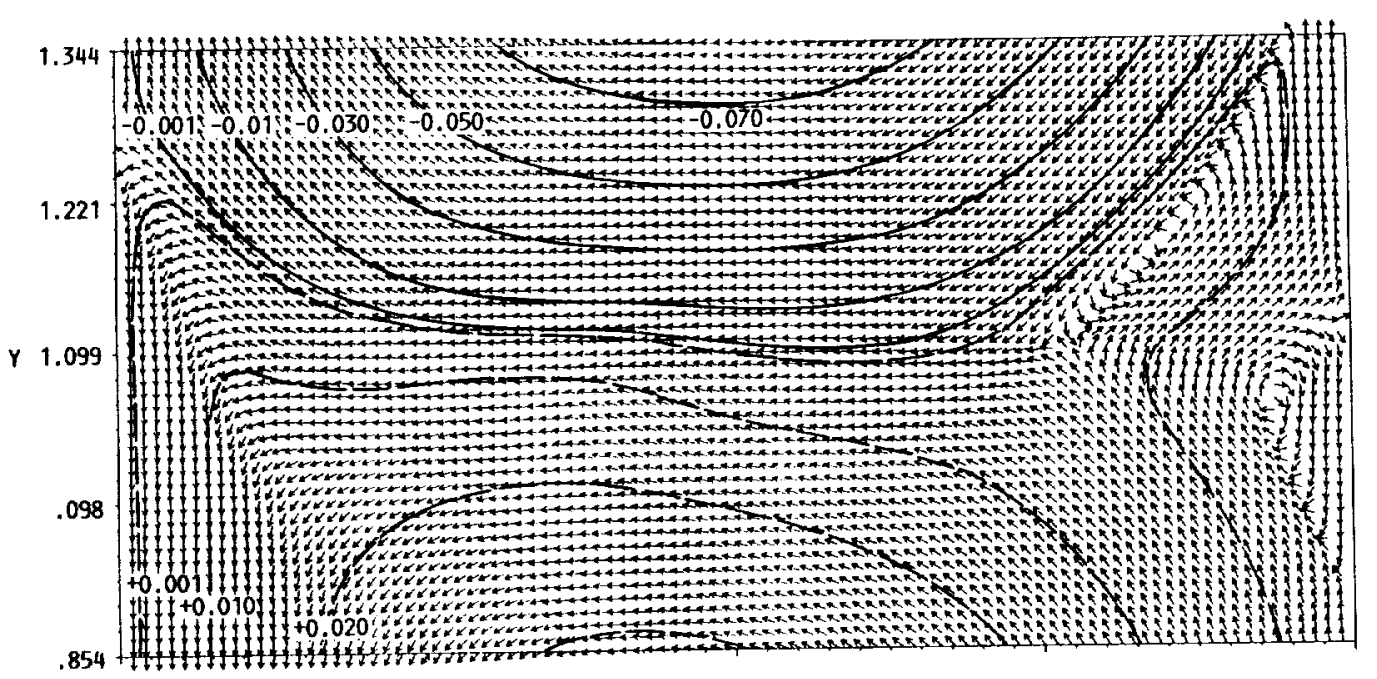

(D) $\mathrm{t}=4100.75$.

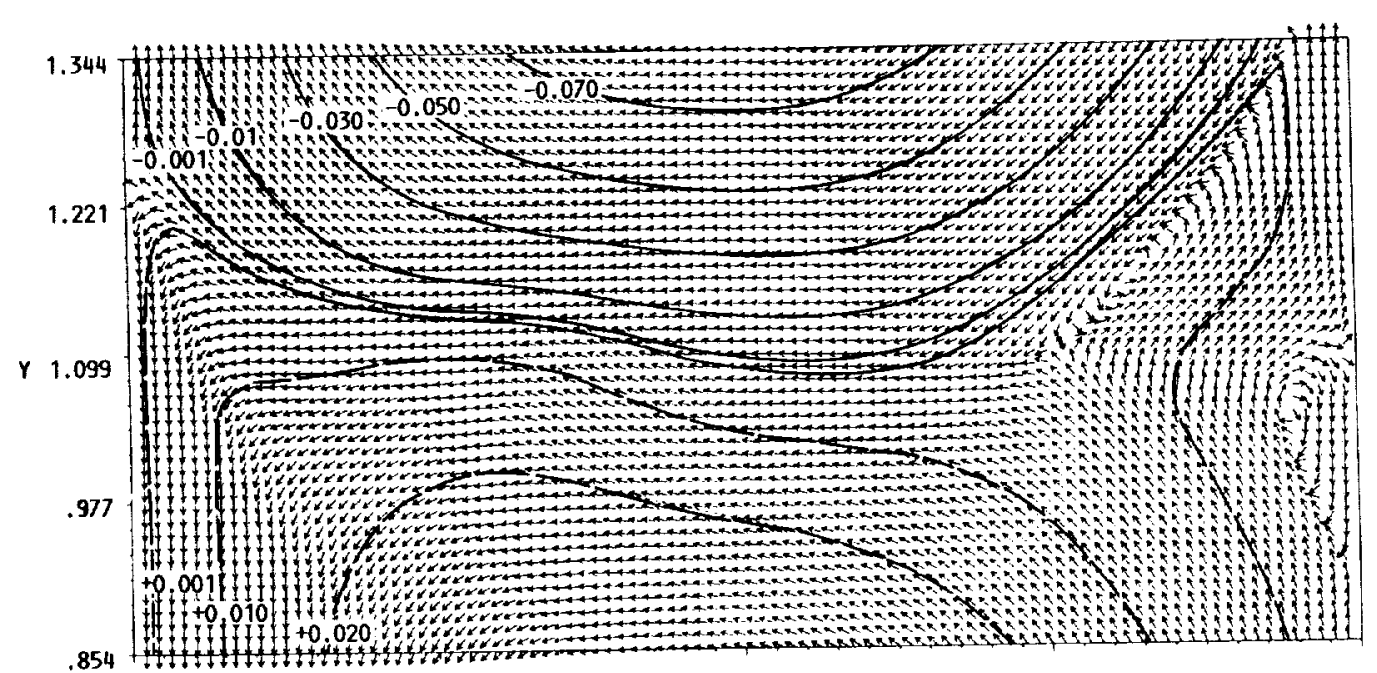

(E) $t=4101.00$.

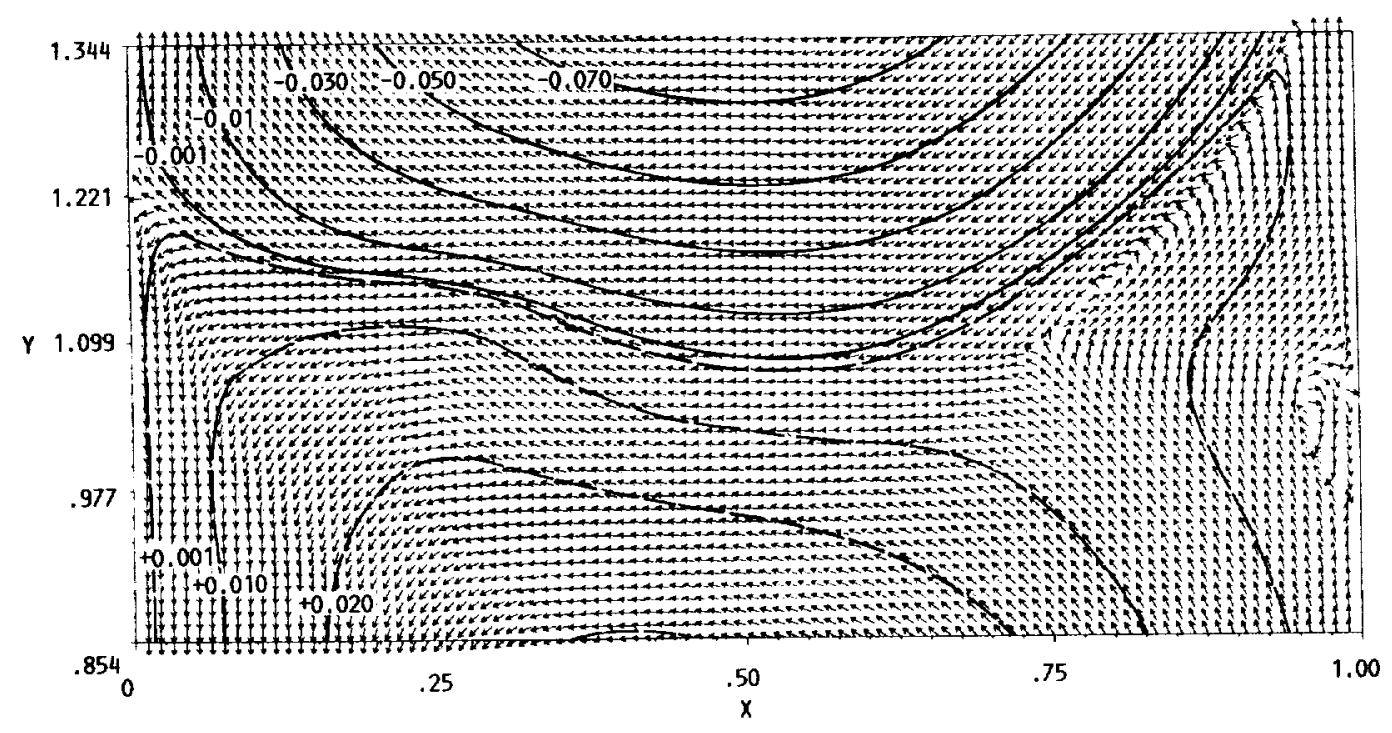

(F) $t=4101.25$.

FIGURE 9. - CONTINUED. 


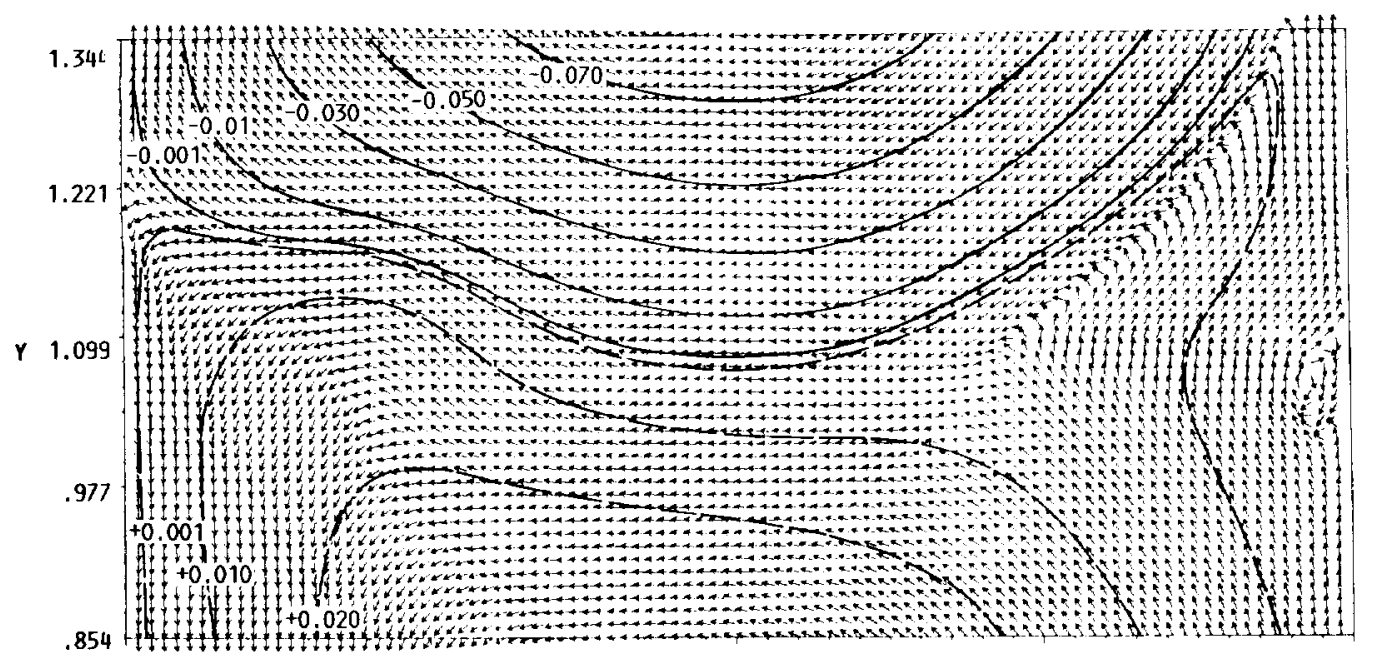

(G) $1=4101.50$.

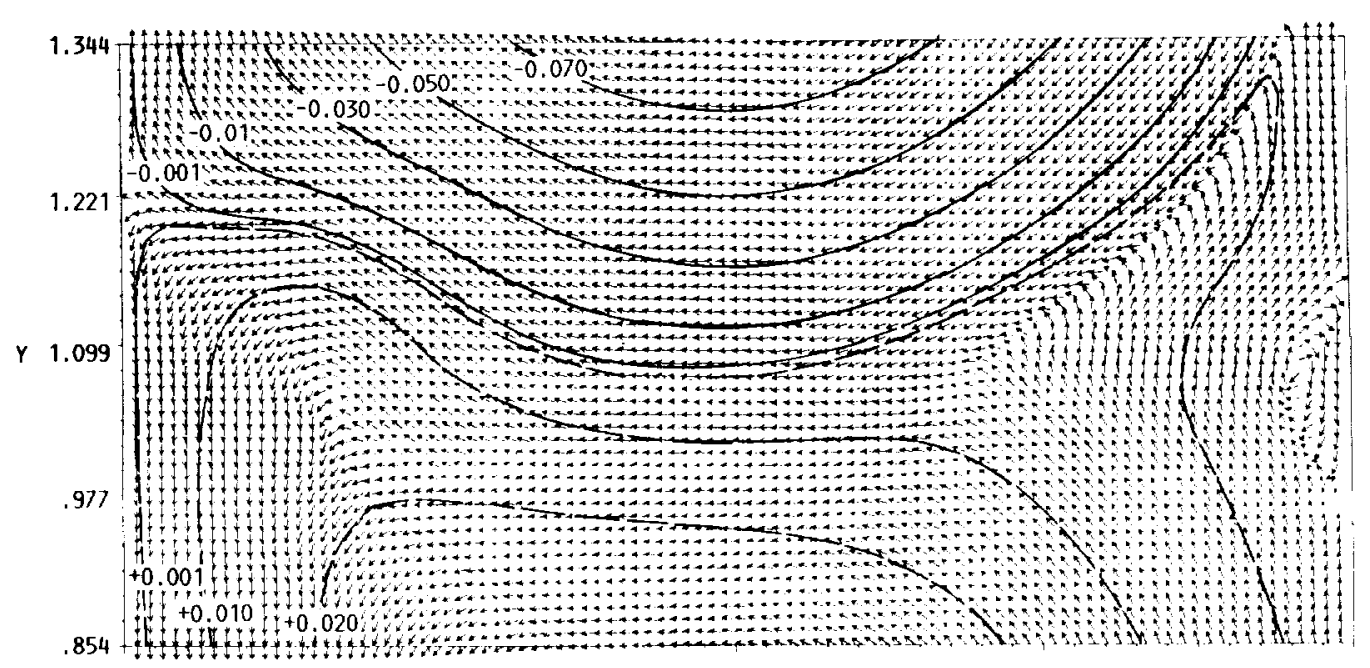

(H) $\mathrm{t}=4101.75$.

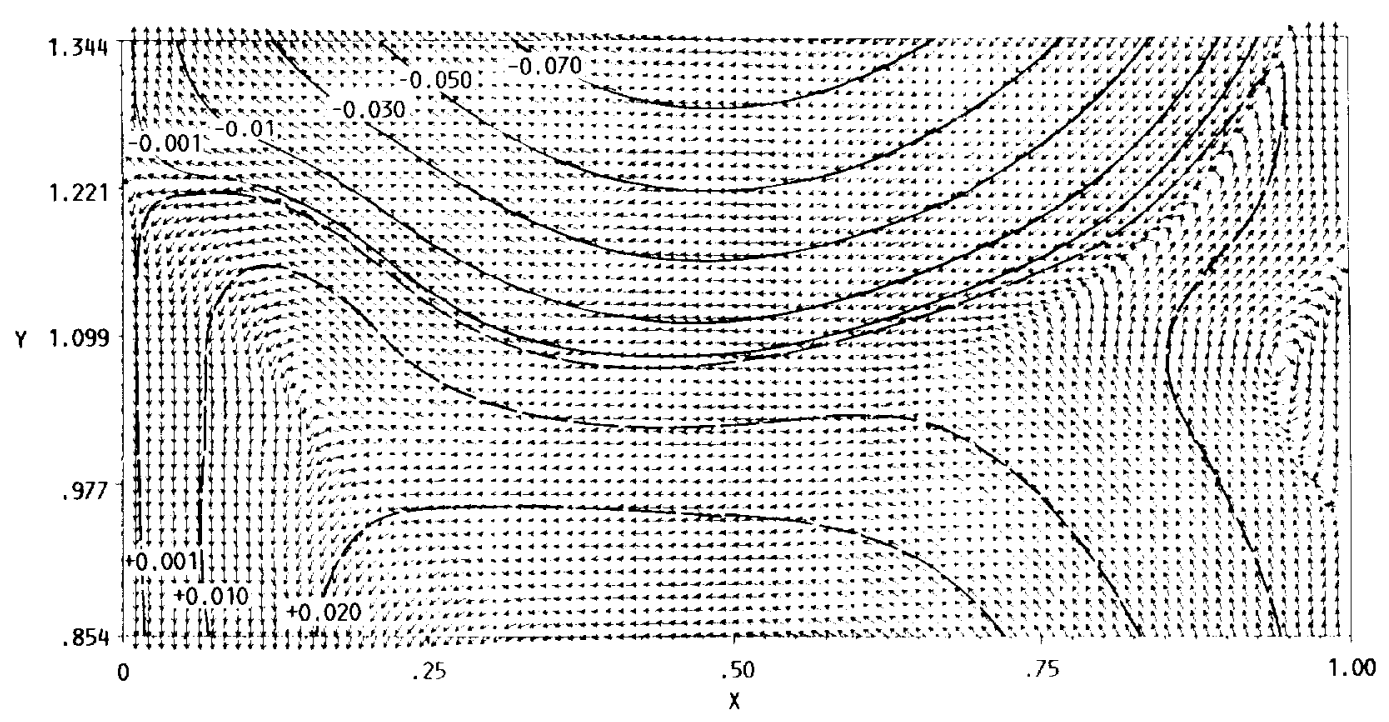

(I) $t=4102.00$.

I. IgURE 9. - CONTINUED. 


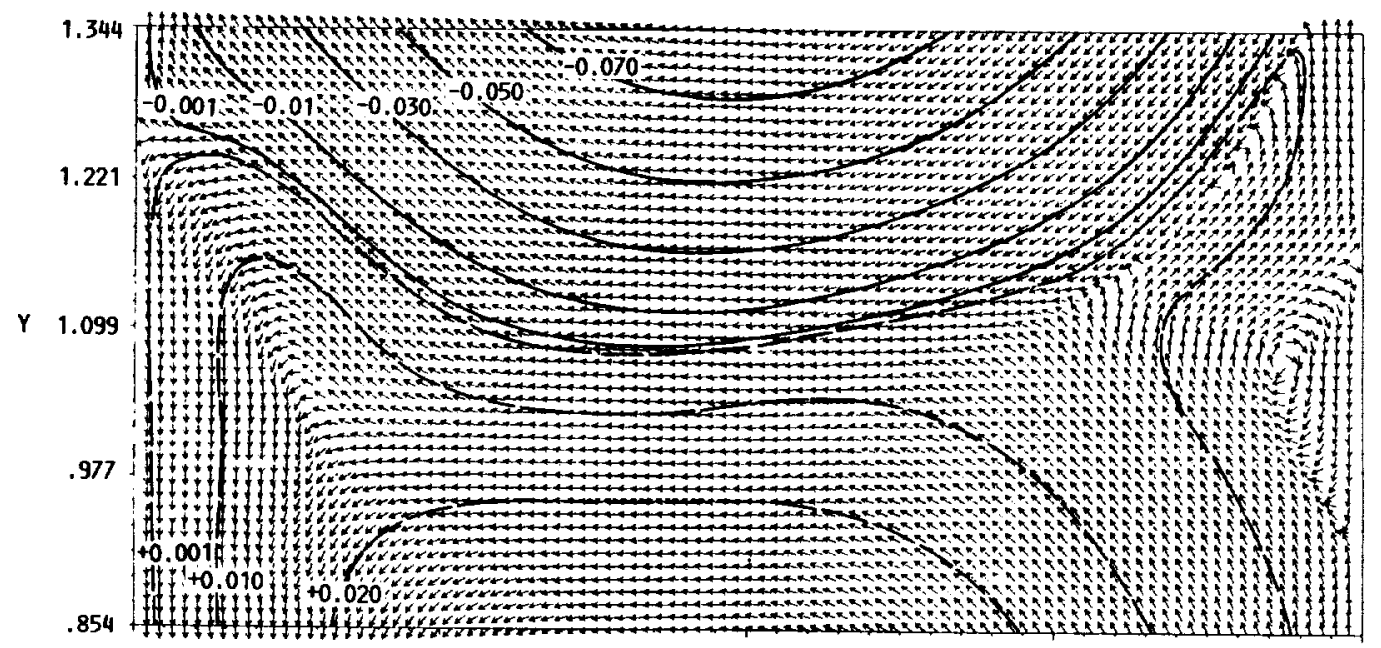

(J) $t=4102.25$.

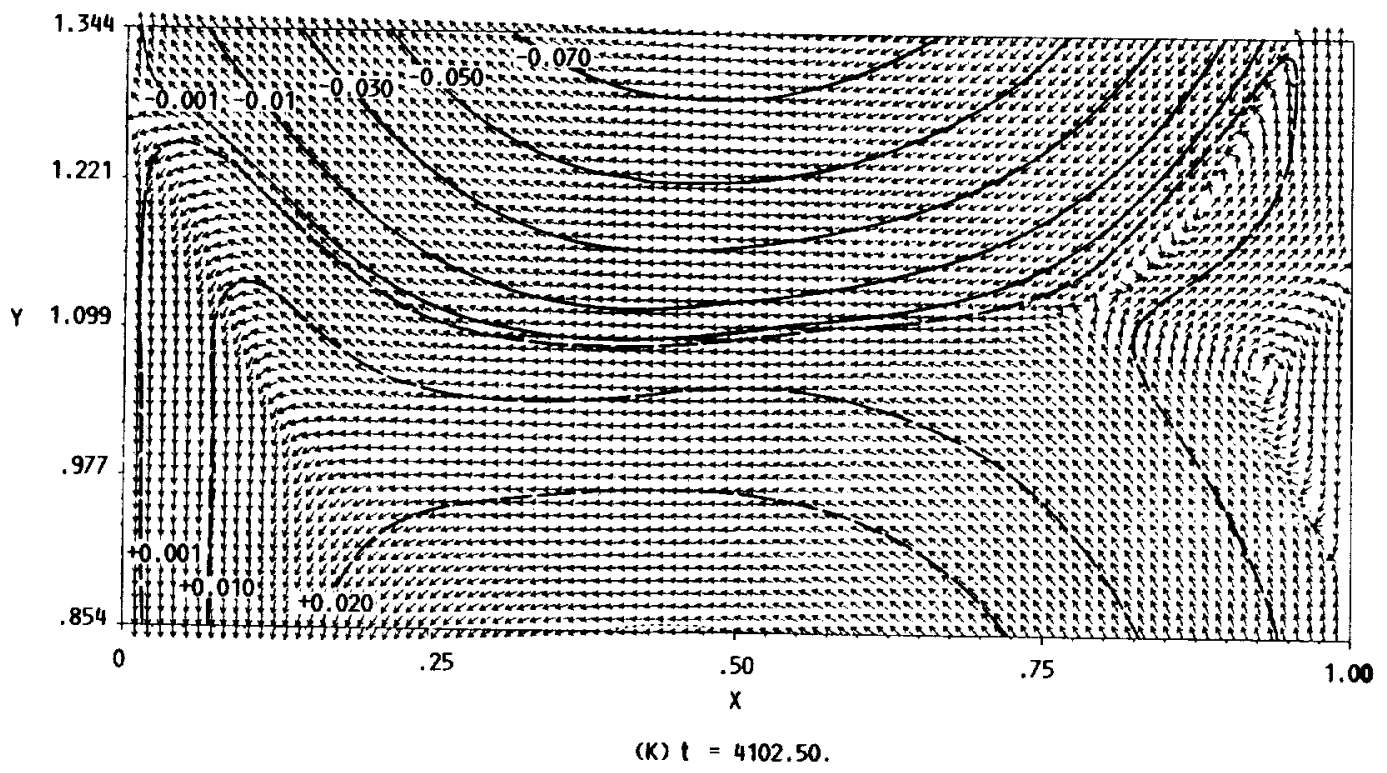

FIGURE 9. - COMCLUDED. 


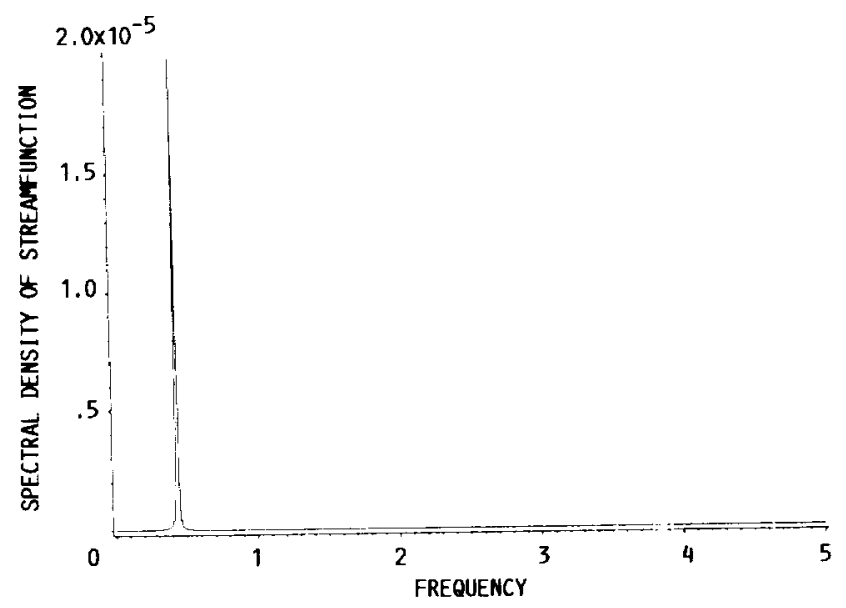

(A) SPECTRAL DENSITY FOR STREAMFUNCTION.

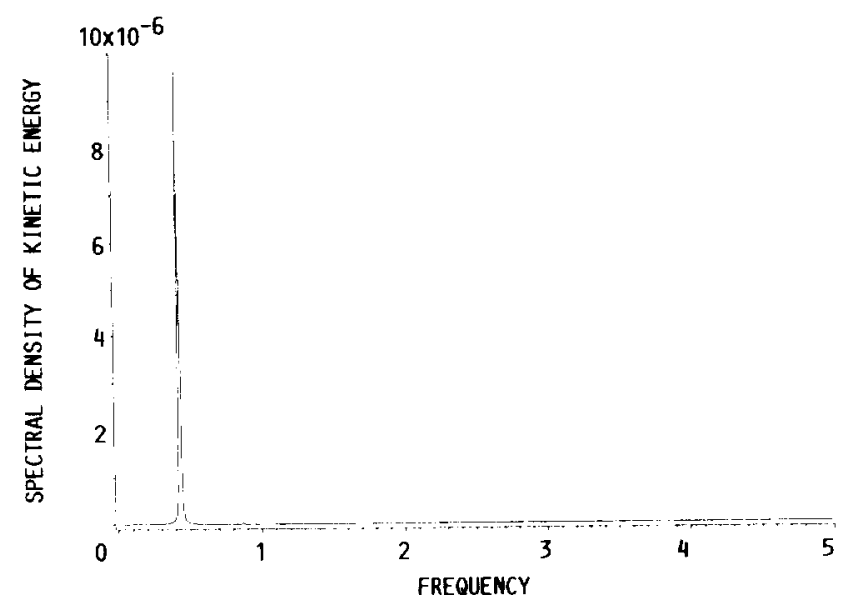

(B) SPECTRAL DENSITY FOR KINETIC ENERGY.

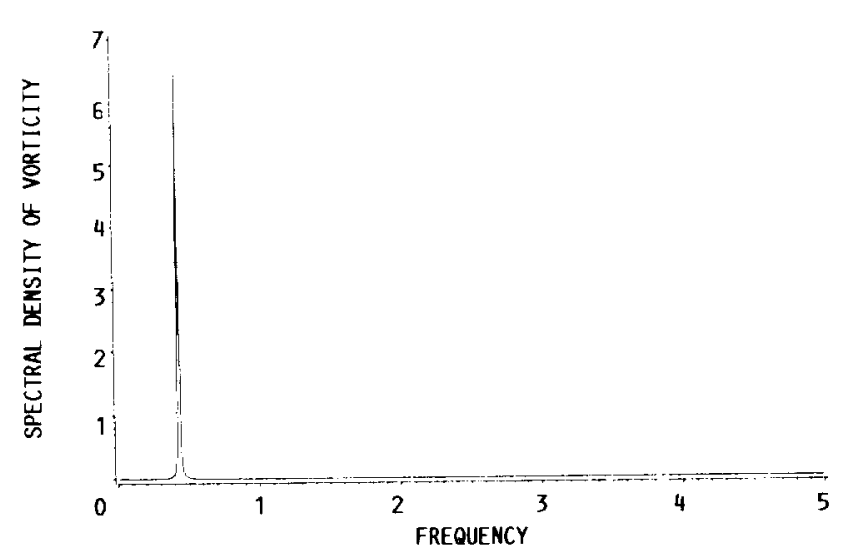

(C) SPECTRAL DENSITY FOR VORTICITY.

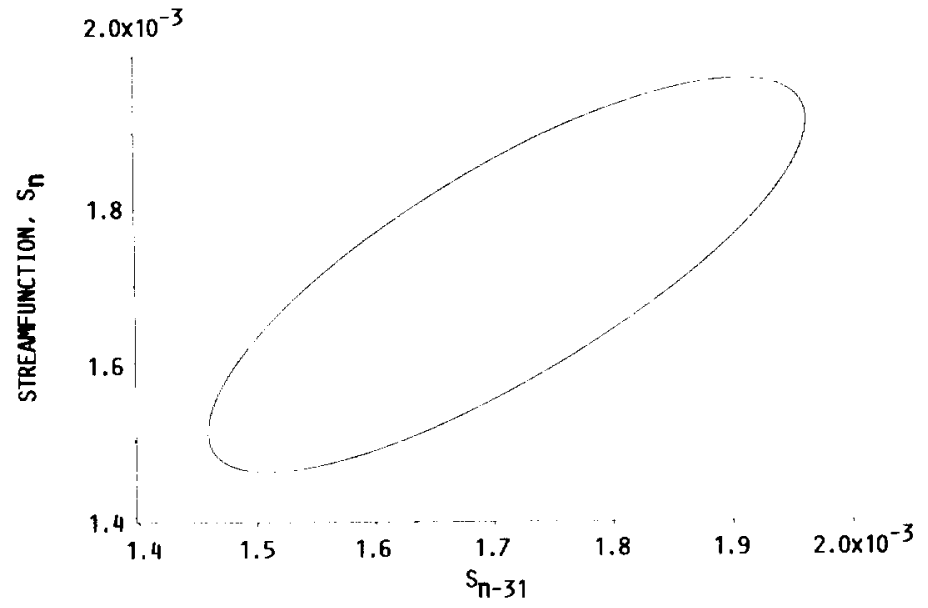

(D) STREAMFUNCTION PHASE PORTRAIT, 31 TIME STEP LAG.

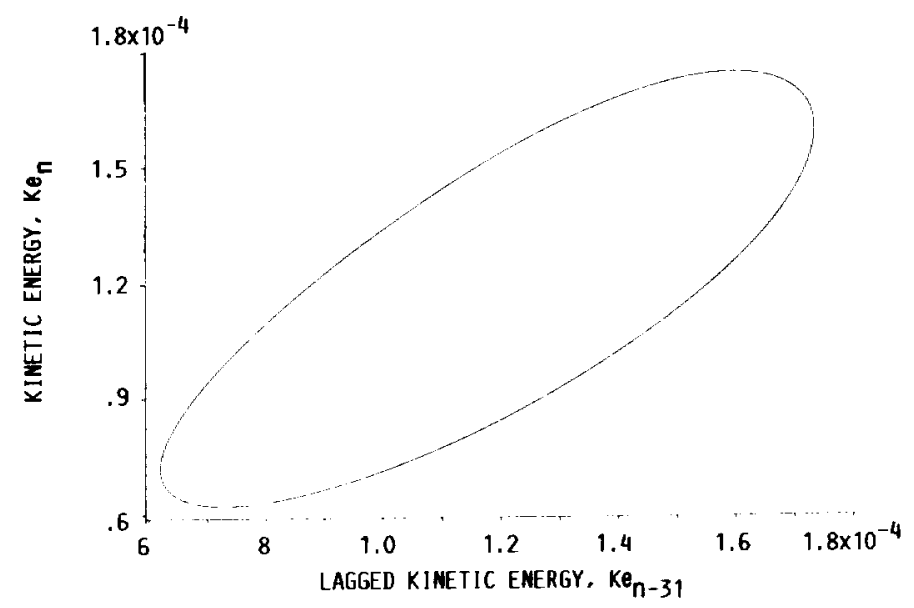

(E) KINETIC ENERGY PHASE PORTRAIT, 31 TIME STEP LAG.

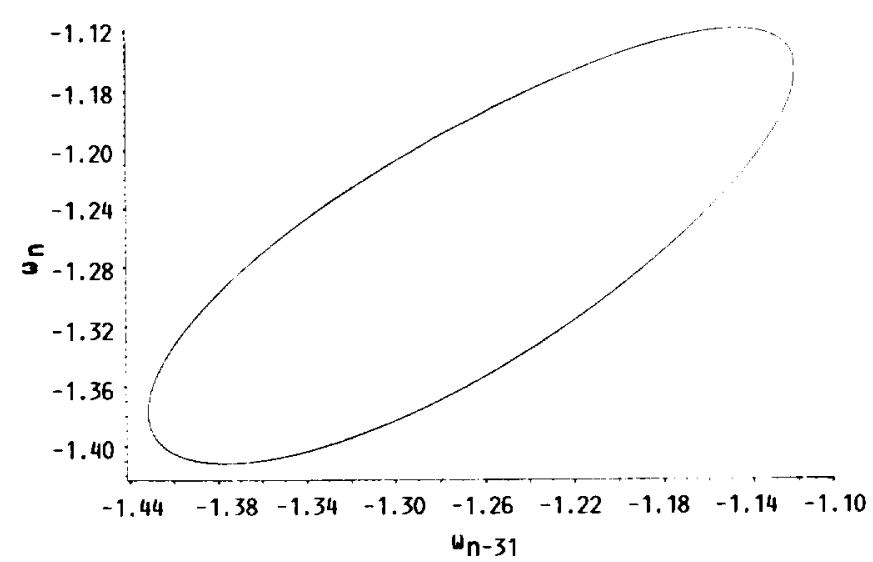

(F) VORTICITY PHASE PORTRAIT, 31 TIME STEP LAG.

FIGURE 10. - DATA AT $(\mathbf{i}, j)=(88,120)$ FOR $4000<1 \leq 4100$, WITH Re $=5000$ ON A $96 \times 192$ GRID. 


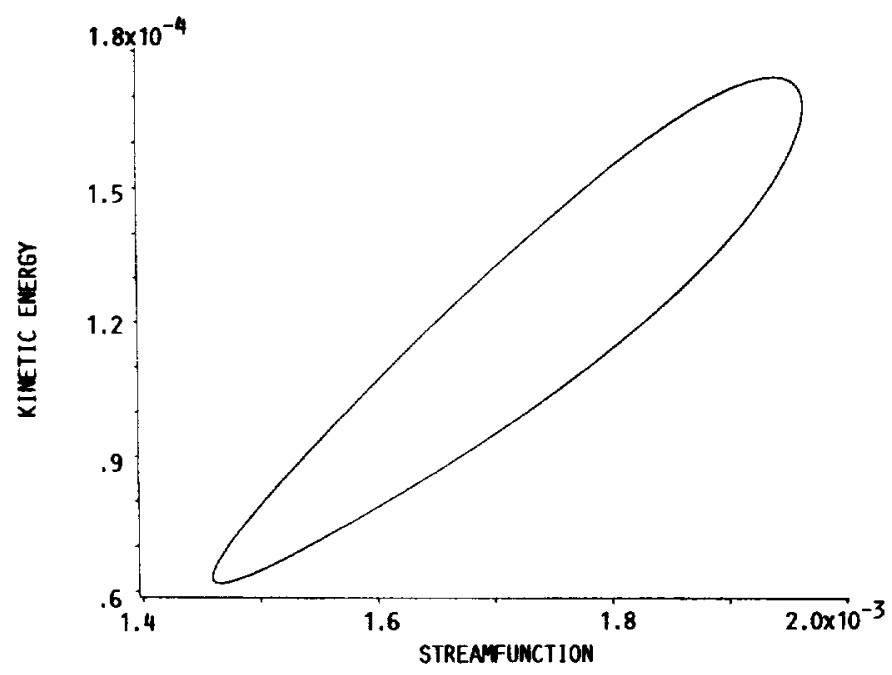

(G) STREAMUNCTION VERSUS KIMETIC ENERGY.

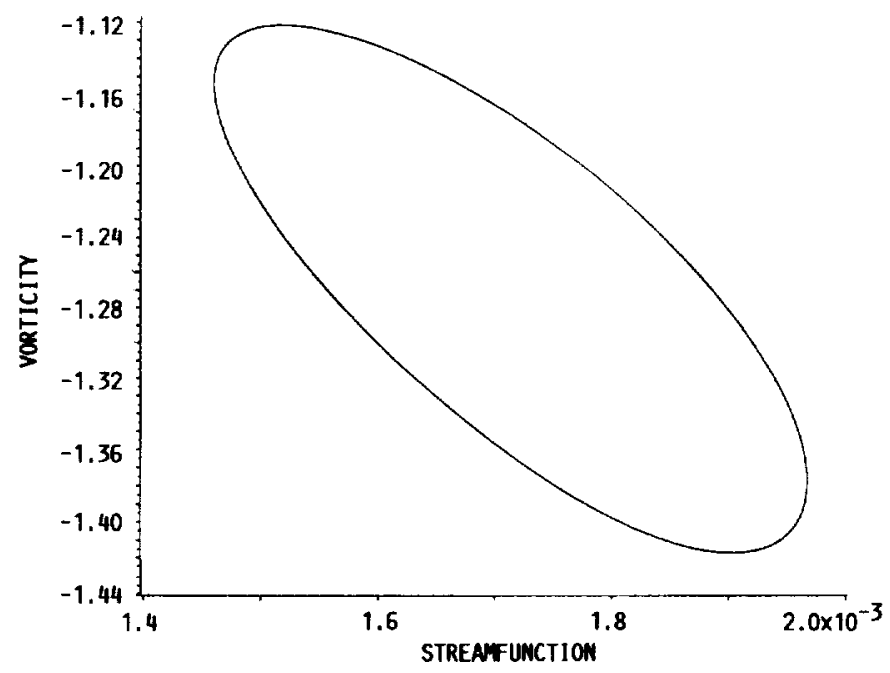

(H) STREAMFUMCTION VERSUS VORTICITY,

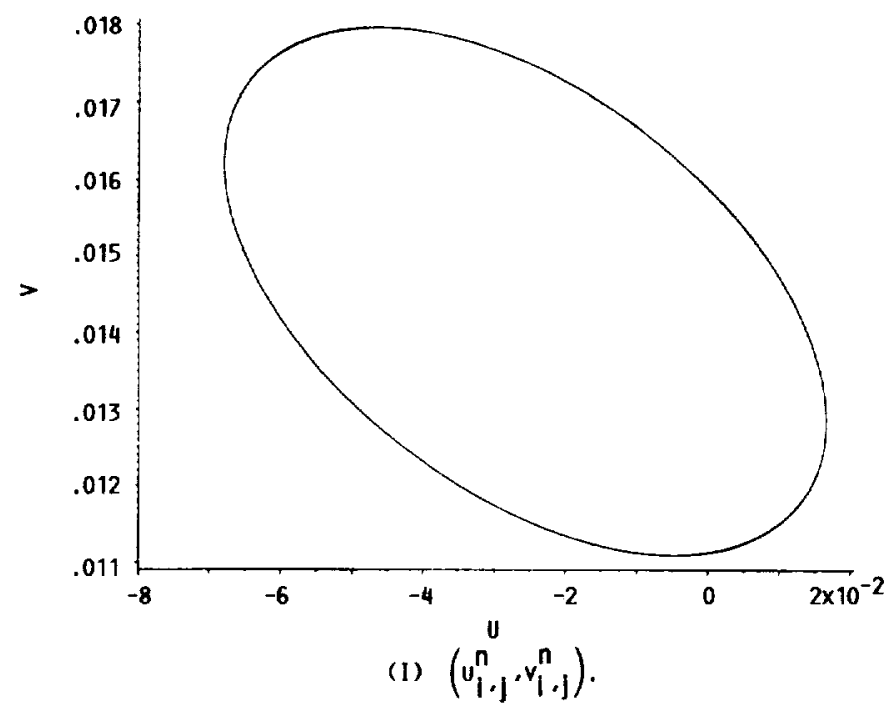

FIGURE 10. - CONCLUDED. 


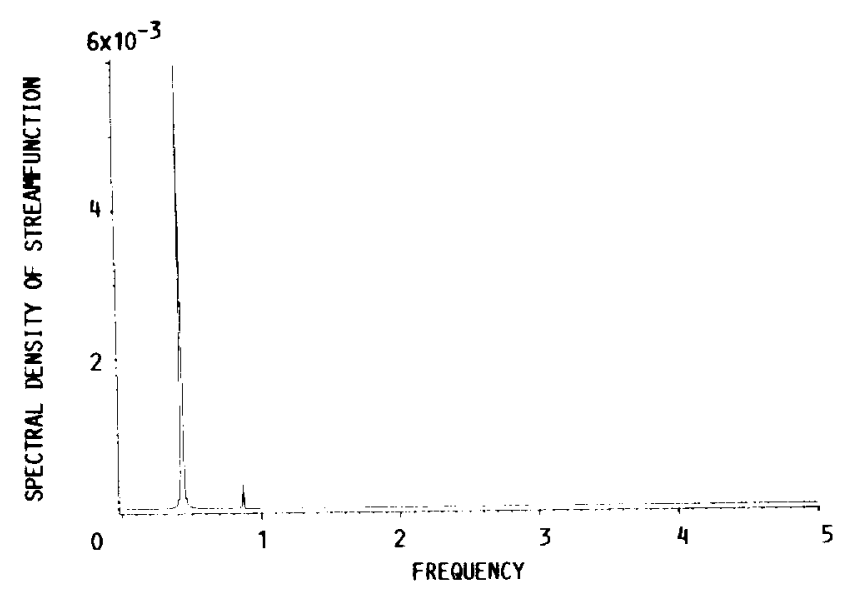

(A) SPECTRAL DENSITY FOR STREAMFUNCTION.

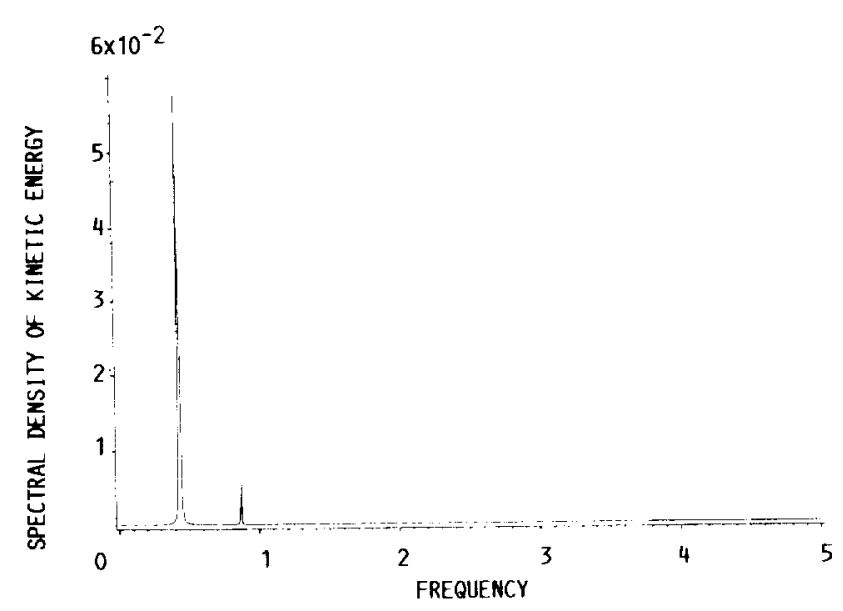

(B) SPECTRAL DENSITY FOR KINETIC ENERGY.

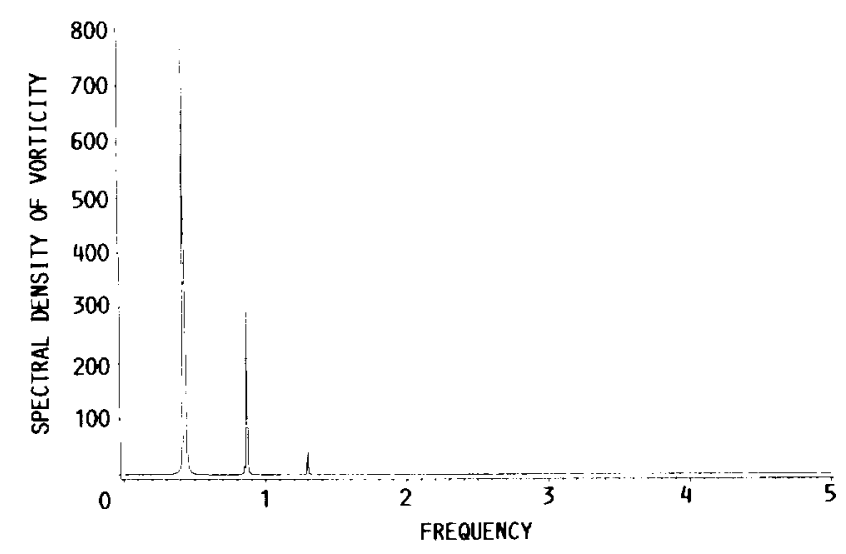

(C) SPECTRAL DENSITY FOR VORTICITY.

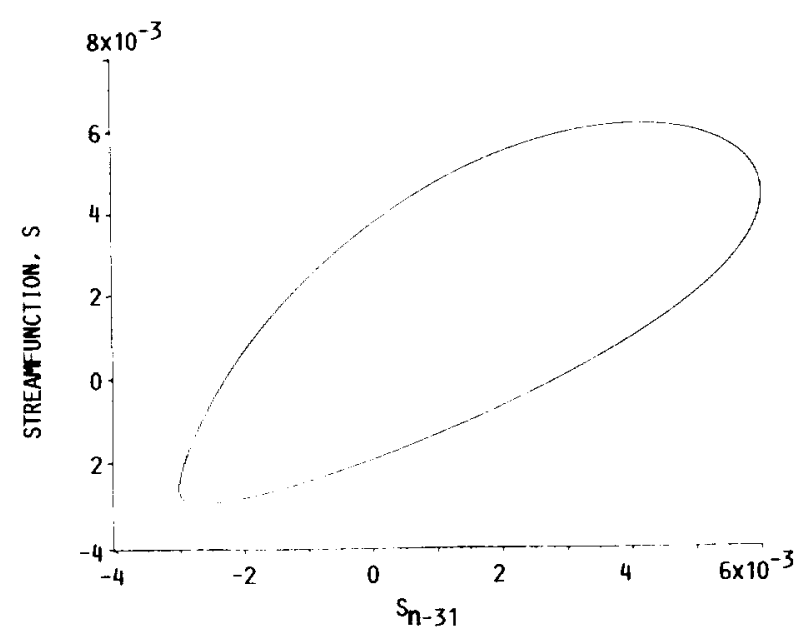

(D) STREAMFUNCTION PHASE PORTRAIT, 31 TIME STEP LAG.

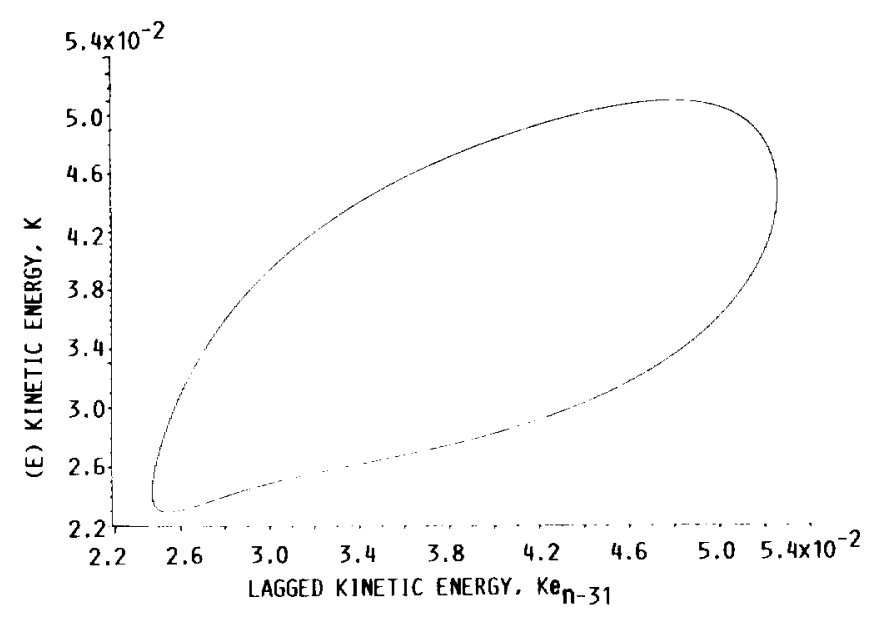

(E) KINETIC ENERGY PHASE PORTRAIT, 31 TIME STEP LAG.

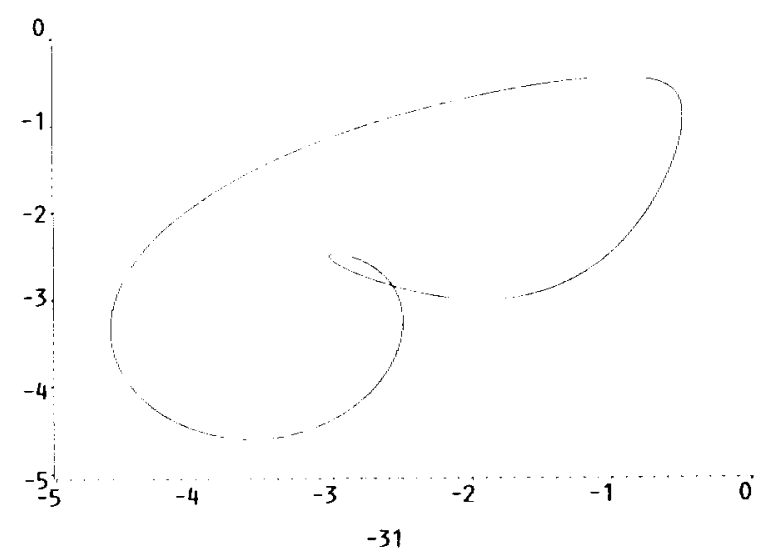

(F) VORTICITY PHASE PORTRAIT, 31 TIME SIEP LAG.

FIGURE 11. - DATA AT $(i, j)=(32.106)$ FOR $4000<t \leq 4100$. WITH Re $=5000$ ON A $96 \times 192$ GRID. 


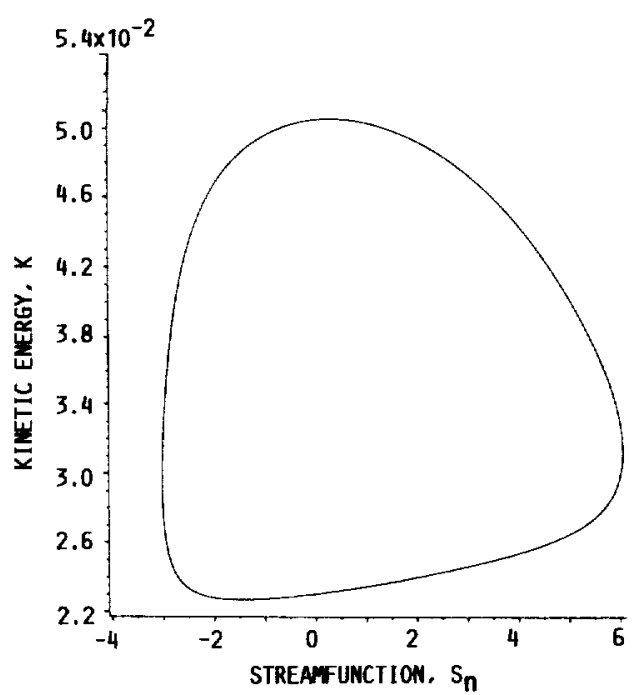

(G) STREAMFUNCTION VERSUS KIMETIC EMERGY.

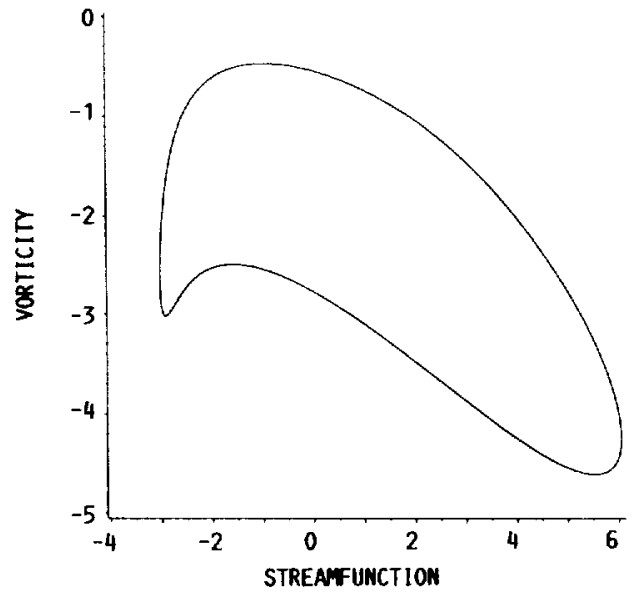

(H) STREAMFUNCTION VERSUS VORTICITY.

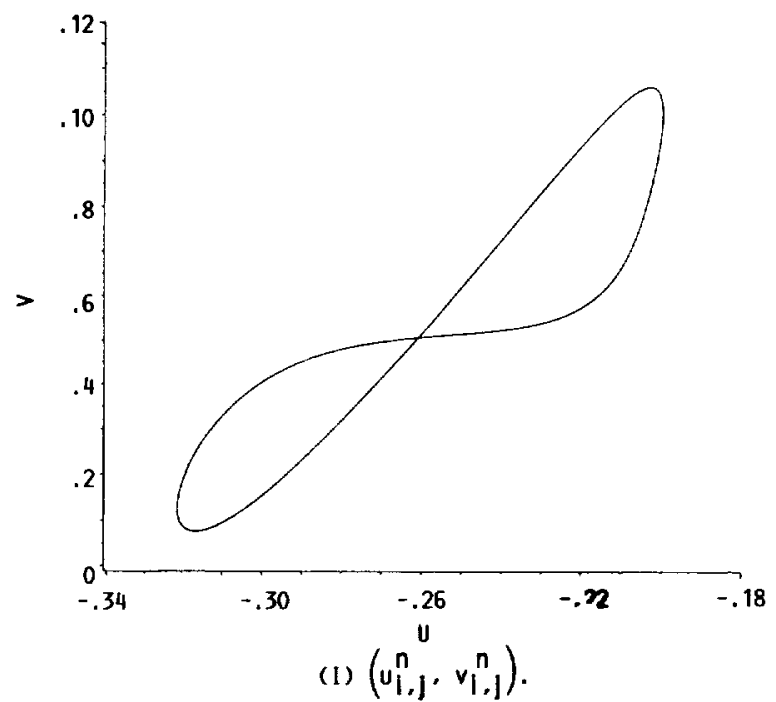

FIGURE 11. - CONCLUDED. 


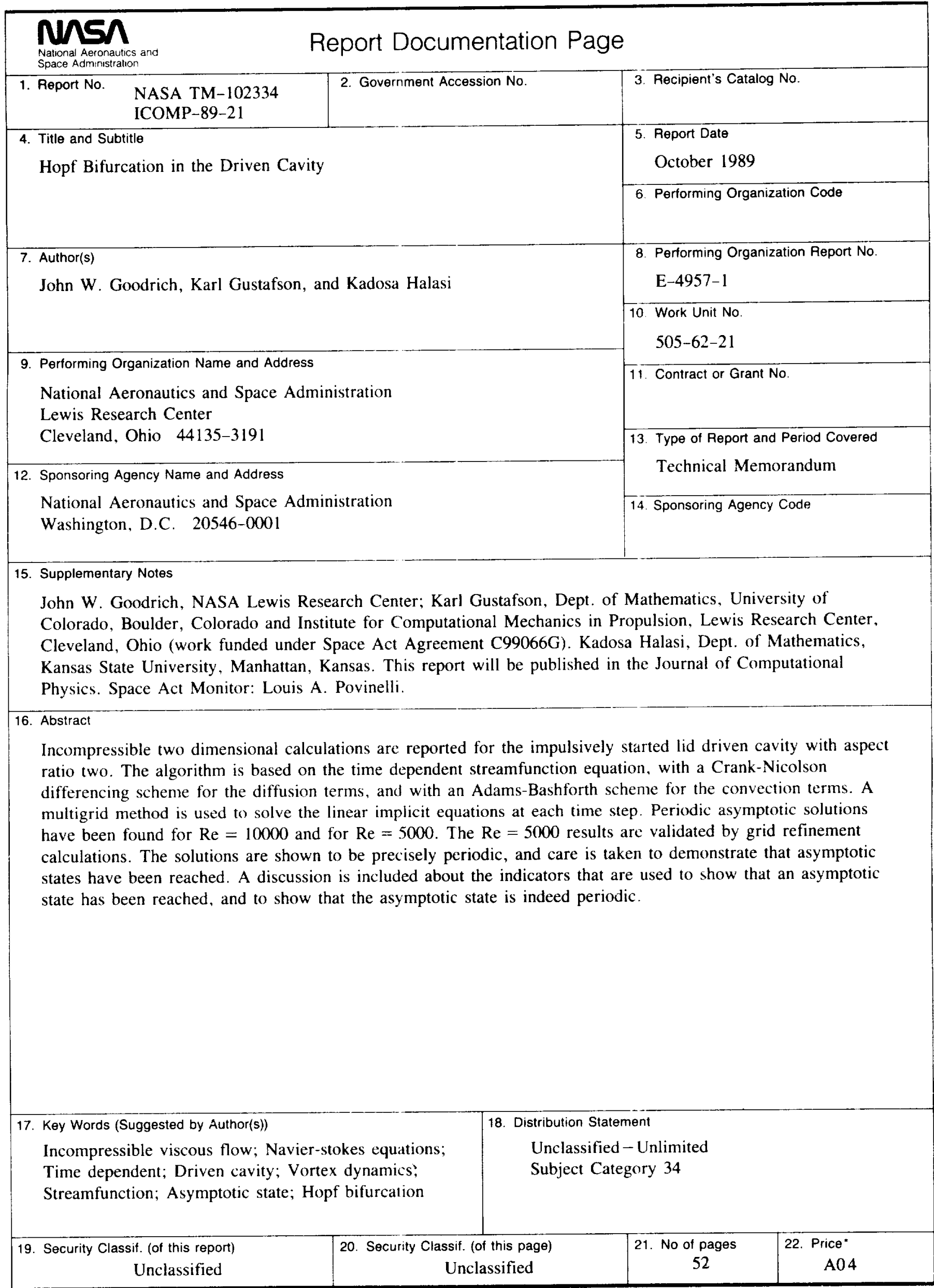




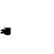

